\title{
Data Driven Intelligent Grid Stability Monitoring and Adaptive Emergency Response
}

\author{
Hasan Ul Banna \\ West Virginia University, hu0001@mix.wvu.edu
}

Follow this and additional works at: https://researchrepository.wvu.edu/etd

Part of the Power and Energy Commons

\section{Recommended Citation}

Ul Banna, Hasan, "Data Driven Intelligent Grid Stability Monitoring and Adaptive Emergency Response" (2019). Graduate Theses, Dissertations, and Problem Reports. 7389.

https://researchrepository.wvu.edu/etd/7389

This Dissertation is protected by copyright and/or related rights. It has been brought to you by the The Research Repository @ WVU with permission from the rights-holder(s). You are free to use this Dissertation in any way that is permitted by the copyright and related rights legislation that applies to your use. For other uses you must obtain permission from the rights-holder(s) directly, unless additional rights are indicated by a Creative Commons license in the record and/ or on the work itself. This Dissertation has been accepted for inclusion in WVU Graduate Theses, Dissertations, and Problem Reports collection by an authorized administrator of The Research Repository @ WVU.

For more information, please contact researchrepository@mail.wvu.edu. 
Hasan UI Banna

Follow this and additional works at: https://researchrepository.wvu.edu/etd

Part of the Power and Energy Commons 


\section{Data Driven Intelligent Grid Stability Monitoring and Adaptive Emergency Response}

\section{Hasan UI Banna}

Dissertation submitted

to the Benjamin M. Statler College of Engineering and Mineral Resources at West Virginia University

in partial fulfillment of the requirements for the degree of

Doctor of Philosophy in

Electrical Engineering

Muhammad A. Choudhry, Ph.D.

Hong-Jian Lai, Ph.D.

Yanfang Ye, Ph.D.

Jignesh Solanki, Ph.D.

Sarika Khushalani Solanki, Ph.D., Chair

Lane Department of Computer Science and Electrical Engineering

Morgantown, West Virginia

2019

Keywords: Low Frequency Oscillations, Phasor Measurement Unit, Ensemble Learning, Coherence Identification, Dynamic Time Warping, Controlled Islanding Copyright 2019 Hasan Ul Banna 


\begin{abstract}
Data Driven Intelligent Grid Stability Monitoring andAdaptive Emergency Response by

Hasan Ul Banna
\end{abstract}

Power grids are large cyber-physical systems with physical operation controlled and integrated through communications. The technological breakthroughs made by the availability of low-cost, high speed communications, larger storage spaces, greater internet bandwidths have led to increased attentions towards advantages of these systems. The use of Phasor Measurement Unit (PMU) in complex interconnected power system for monitoring and control has increased significantly. This has resulted in huge amounts of data and growing databases, thus gearing towards an era where utilities might encounter an enormous amount of data daily from their measurement units including sensitive information useful for daily operations. With increasing energy demand, more energy resources are continuously being added in the existing network. This complex network thus operates comparatively closer to its stability limits with minimal flexibility and reliability. Such conditions may lead to low amplitude oscillations causing power fluctuations and discontinuity of supply in some cases. Therefore, recently, the attention of utilities has shifted towards tools and methods, which help in locating the source of these electromechanical oscillations. We propose a novel data driven Credibility Search Ensemble Learning (CSEL) technique to identify the source location of these oscillations using synchrophasor measurements, offline credibility estimation and data mining based classification models. The proposed framework was tested and validated with promising results using western interconnection power system in North America (WECC-179). The reliability and robustness of the proposed framework was checked against measurement errors in PMUs as well as for practical topology change scenarios. Such oscillation source identification methods were mostly developed and tested in transmission system, where PMU measurements at almost each bus are readily available. In addition, the presence of inertia in the form of rotating synchronous machines is also extremely helpful against these oscillations. On the other hand, with increased penetration of DERs, the analysis of these oscillations in islanded microgrid, which is not connected or supported through large interconnected transmis- 
sion system, is very crucial. We demonstrate and validate the applicability of the proposed model free source identification approach for 13-node and 34-node distribution networks operating in islanded mode. We also analyze the performance with multiple causes and also with the cause being a fluctuating load.

In events, where finding the oscillation source of disturbance does not provide adequate information to the system operator to take countermeasures, controlled islanding can still be applied as a last countermeasure to prevent system-wide instabilities and blackouts. It splits the system into self-sustained islands to maintain transient stability at the expense of possible loss of load. However, the stability of each newly formed island depends on the coherency of the generating units. Generator coherence identification is critical to controlled islanding scheme as it helps identify the optimal cut-set to maintain the transient stability of the post-islanding systems. Therefore, correct and adaptive identification of generator's coherency is essential. Moreover,the coherency between groups of generators varies over time, due to changing network topology and operating conditions, necessitating real-time coherency determination. We propose a novel approach for online generator coherency identification using phasor measurement unit (PMU) data and dynamic time warping (DTW). In addition, we also propose a unique data driven approach for coherence identification of generators using Phasor Measurement Unit (PMU) data and its structural characteristic measures like Kurtosis, entropy etc. Results from the coherence identification are used to further cluster nongenerator buses using spectral clustering with the objective of minimizing power flow disruptions. The proposed approach is validated and compared to existing methods on the IEEE 39-bus system and WECC 179-bus system, through which its advantages are demonstrated. 


\section{ACKNOWLEDGEMENT}

Many people encouraged and supported me during my stay at West Virginia University. First of all, with immense gratitude and pleasure, I wish to express my sincere thanks to my mentor and advisor Dr. Sarika Khushalani Solanki. Without her continuous motivation, this research would not have been successfully completed. It is due to her persistent support and guidance that I made huge progress towards improving my writing and presentation skills as a researcher.

Besides my advisor, I have the greatest respect for my co-advisor Dr. Jignesh Solanki for his invaluable input into my research. I would like to thank Dr. Muhammad A. Choudhry who has inspired and guided me throughout my Ph.D. study. I would also like to thank Dr. Hong-Jian Lai and Dr. Yanfang Ye for serving on my P.D. examination and evaluation committee and sharing their knowledge and experience.

I like to acknowledge the support rendered by my friends and lab mates in many sleepless nights for working together before deadlines; and for stimulating discussions and memories during conferences. I was very fortunate to have such friends with enormous positive energy.

I would also like to thank my parents and my brother for giving me strength and confidence in pursuit for my doctoral degree. Last but not the least, I would like to thank my wife for her encouragement in the most difficult time and supporting me through this entire journey. 


\section{TABLE OF CONTENTS}

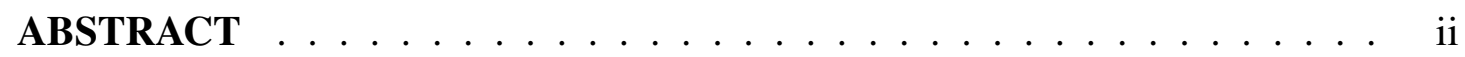

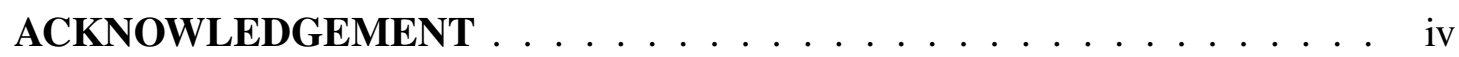

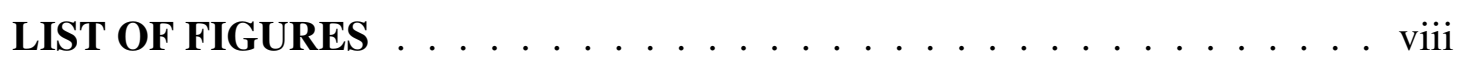

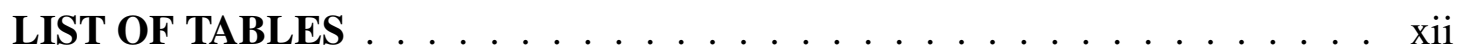

1 Introduction 1

2 Oscillation Source Identification Using Data Driven Credibility Search Ensemble Learning 9

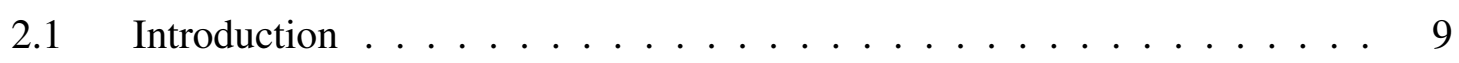

2.2 Classification Models for Oscillation Source Location . . . . . . . . . . 10

2.3 Overview of Proposed Approach . . . . . . . . . . . . . . . . 12

2.3.1 Proposed Credibility search based Oscillation Source Identification 13

2.4 Performance Evaluation Indices . . . . . . . . . . . . . . . . . . . 16

2.4.1 Measurement Error Handling . . . . . . . . . . . . . . . . 16

2.4.2 Topology Changes Impact $\ldots \ldots \ldots$

2.5 Credibility Search of Classification Models _. . . . . . . . . . 17

2.5 .1 Test System . . . . . . . . . . . . . . . . . . . . . . 17

2.5 .2 Training Data $\ldots \ldots \ldots \ldots \ldots \ldots \ldots \ldots$

2.5.3 Selection of most Credible Classification Models _ . . . . . . 18

2.6 Oscillation Source Identification Results for WECC 179-System . . . . 19

2.6 .1 Additional Database Generation _ . . . . . . . . . . . . 20

2.6 .2 Identification Results . . . . . . . . . . . . . . . . . 20

2.6.3 Impact of Topology Changes . . . . . . . . . . . . . . 21

2.6.4 Impact of Measurement Errors _ . . . . . . . . . . . . . 21

2.6.5 Comparison with Average Weight based Approach _. . . . . . 22

2.7 Discussion . . . . . . . . . . . . . . . . . . . 22 
2.8 Evolution of Oscillations in Islanded Microgrid . . . . . . . . . . . . 23

2.9 Source identification Results in Islanded Microgrid . . . . . . . . . . . 27

2.9.1 Single oscillation source at the PV generator . . . . . . . . . . 28

2.9.2 Multiple oscillation sources at PV generators . . . . . . . . . 32

2.9.3 Oscillation source at highly fluctuating cyclic load . . . . . . . 36

2.9.4 Performance in the presence of ambient noise . . . . . . . . . . 40

2.10 Conclusion . . . . . . . . . . . . . . . . . . 40

3 Data Driven Coherence Identification using Dynamic Time Warping for Controlled Islanding $\quad 42$

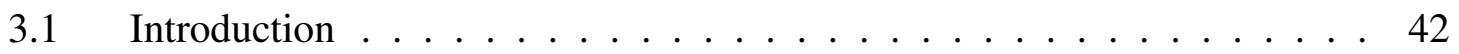

3.2 Adaptively controlled islanding framework . . . . . . . . . . . . . . 44

3.2.1 Dynamic Time Warping (DTW) based Generator Coherency Identification ....................... 45

3.2.2 Buses clustering for controlled islanding . . . . . . . . . . . . 47

3.2 .3 Discussion . . . . . . . . . . . . . . . 49

3.3 Simulation Results and Performance Evaluation . . . . . . . . . . . . 50

3.3.1 Case Study 1: Comparison with Correlation based Method . . . . 52

3.3.2 Case Study 2: Comparison with Community detection method . . 56

3.3.3 Western interconnection power system of North America . . . . . 62

3.4 Performance of proposed approach with partial observability and noise . . 64

3.5 Conclusion . . . . . . . . . . . . . . . . 66

4 Coherence Identification using Structural Characteristic Measures of $\begin{array}{lr}\text { PMU Time Series Data } & 67\end{array}$

4.1 Introduction . . . . . . . . . . . . . . . . . 67

4.2 Characteristic Measures of Trajectories for Coherency Identification . . . 68

4.2.1 Skewness . . . . . . . . . . . . . . . . 68

4.2 .2 Self-Similarity . . . . . . . . . . . . . . . . 69

4.2 .3 Kurtosis ..................... 70

4.2 .4 Approximate Entropy . . . . . . . . . . . . . . . . 71

4.3 Proposed Cumulative Characteristic Measure based Coherency Identifi-

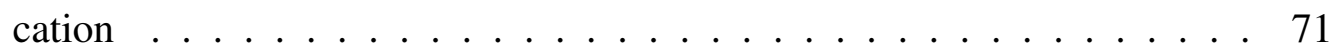


4.4 Experimental Validation and Performance Evaluation . . . . . . . . . 73

4.4.1 Experimental Validation . . . . . . . . . . . . 75

4.4.2 Performance Comparison . . . . . . . . . . . . . 75

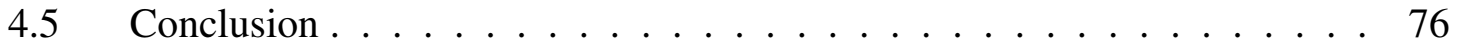

$\begin{array}{lll}5 & \text { Recommendations for Future Work } & 77\end{array}$

LIST OF PUBLICATIONS . . . . . . . . . . . . . . . . . . . 79

REFERENCES . . . . . . . . . . . . . . . . . 80

Appendices

Appendix A Example: DTW based Similarity Evaluation 95 


\section{LIST OF FIGURES}

1.1 Wide-Area forced oscillations resulting in variations of the system fre-

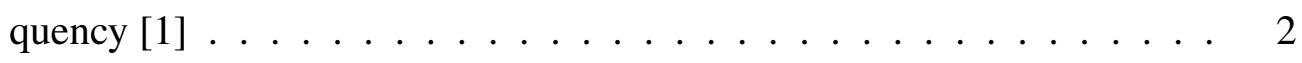

1.2 Voltage variations during $3 \mathrm{~Hz}$ oscillation event at wind power plant [2] . 6

1.3 Generator response following cascaded outages . . . . . . . . . 8

2.1 Mapping of input vector to higher dimensional feature space using kernel function . . . . . . . . . . . . . . . 10

2.2 Learning process from available measurements to the desired source location .......................... 11

2.3 Majority voting leading towards erroneous prediction . . . . . . . 13

2.4 Implementation flow of proposed credible search ensemble learning approach; green box: offline validation stage, red box: online prediction stage .............................. 16

2.5 Three phase short circuit fault at bus 159 in WECC 179-bus system . . . 18

2.6 Prediction accuracy for each individual classification models . . . . . 19

2.7 Box plot of missclassification rate for individual classification models $\quad$. 19

2.8 Missclassification rate for individual reported features . . . . . . . . . 20

2.9 Correlation maps for rotor angles (left) G11-source of oscillations, (center) G4-source of oscillations, (right) G11-source of oscillations for

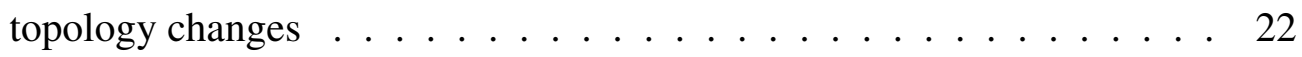

2.10 Time ranges of dynamic phenomenons in transmission system . . . . 23

2.11 Droop characteristics of SGs . . . . . . . . . . . . . . . 24

2.12 Power controller for droop strategy . . . . . . . . . . . . . . 25

2.13 Three bus islanded microgrid [3] . . . . . . . . . . . . 25

2.14 Eigenvalue locus with varying droop gain $\mathrm{k}_{P} \ldots \ldots . \ldots 26$

2.15 Voltage magnitude for different values of $\mathrm{k}_{Q} \ldots \ldots \ldots 26$

2.16 Modified IEEE 13-node distribution network . . . . . . . . . . . . 28 
2.17 Variations in voltage magnitude of photovoltaic based DGs . . . . . . . 29

2.18 Oscillation frequency of voltage magnitude . . . . . . . . . . . . 29

2.19 Distribution of the attained ACC of top three classifier models during credibility search phase for voltage magnitude (a) and angle (b) . . . . . 30

(b) 30

2.20 Modified IEEE 34-node distribution network . . . . . . . . . . . . . 30

2.21 Variations in voltage magnitude of photovoltaic based DGs

2.22 Distribution of the attained ACC of top three classifier models during credibility search phase for voltage magnitude (a) and angle (b) . . . . . 31

(b)

2.23 CD diagrams for the nemenyi tests; Comparing each source identification technique in terms of TPR (a) and FPR (b) for the single oscillation source scenarios

(a)

(b)

2.24 Variations in voltage magnitude of photovoltaic based DGs . . . . . . 33

2.25 voltage magnitude variations for different frequency modes . . . . . . . 33

2.26 Distribution of the attained ACC of top three classifier models during credibility search phase for voltage magnitude(a) and angle (b) . . . . . 34

(b)

2.27 Variations in voltage magnitude of Photovoltaic based DGs 35

2.28 Distribution of the attained ACC of top three classifier models during credibility search phase for voltage magnitude(a) and angle (b) . . . . 35

(b)

2.29 CD diagrams for the nemenyi tests; Comparing each source identification technique in terms of TPR (a) and FPR (b) for multiple sources . . 36 36

(b) 
2.30 Variations in voltage magnitude of photovoltaic based DGs . . . . . . 37

2.31 Coherency based islanding scenario for emergency condition . . . . . 37

2.32 Distribution of the attained ACC of top three classifier models during credibility search phase for voltage magni-tude(a) and angle (b) . . . . 38

(b) 38

2.33 Variations in voltage magnitude of photovoltaic based DGs . . . . . 38

2.34 Distribution of the attained ACC of top three classifier models during credibility search phase for voltage magni-tude(a) and angle (b) . . . . . 39

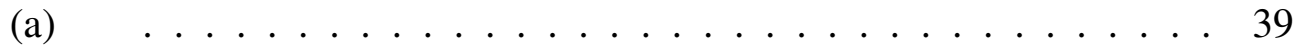

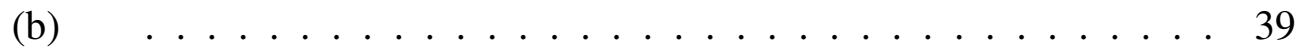

2.35 CD diagrams for the nemenyi tests; Comparing each source identification technique in terms of TPR (a) and FPR (b) for the oscillation caused by fluctuating loads . . . . . . . . . . . . . . . . 39

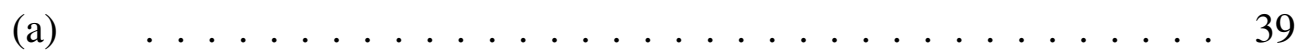

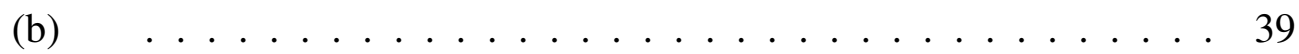

2.36 False positive rate for different measurement noise levels in $\mathrm{dB}$. . . . . 40

3.1 Generators response following cascaded outages . . . . . . . . . . . . . 44

3.2 Optimal warping path . . . . . . . . . . . . . 46

3.3 Algorithm 1 (adaptive controlled islanding) . . . . . . . . . . . 50

3.4 System losing synchronism and becoming unstable . . . . . . . . . 53

3.5 Formation of two islands . . . . . . . . . . . . . . . . 54

3.6 Generators rotor angle responses and voltage profiles at system buses after implementing proposed islanding scheme . . . . . . . . . . 55

3.7 Active power load shedding comparison . . . . . . . . . . 56

3.8 Silhouette plots for coherent generators groups . . . . . . . . . . . 57

3.9 System losing synchronism and becoming unstable $\ldots \ldots$. . . . . 58

3.10 Formation of two islands . . . . . . . . . . . . . . . . . . . 59

3.11 Generators rotor angle responses and voltage profiles at system buses after proposed islanding . . . . . . . . . . . . . 60 60

3.12 Active power load shedding comparison . . . . . . . . . . . . 60

3.13 Silhouette plots for coherent generators groups . . . . . . . . . . . . 61 
3.14 Performance of proposed coherency algorithm with partial loss of PMU

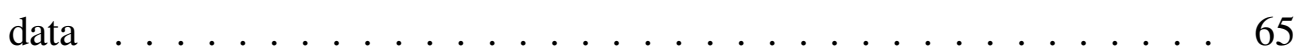

3.15 Generators' coherency grouping identified by proposed coherency method without noise ...................... 65

3.16 Generators' coherency grouping identified by proposed coherency method in the presence of white Gaussian noise . . . . . . . . . 66

4.1 Rotor angle trajectories of generators in WECC 179 system following a disturbance at $0.5 \mathrm{sec} \ldots \ldots \ldots \ldots$

4.2 Three phase short circuit fault at bus 159 in WECC 179-bus system . . . 73

4.3 Rotor angle trajectories of 29 generators in WECC 179 system following a three phase short circuit fault . . . . . . . . . . . . . 74

4.4 Silhouette plots for coherent generator groups . . . . . . . . . . 75

4.5 Coherency quality measure changes as characteristic measures are added 76

5.1 Coherency among inverter based DGs . . . . . . . . . . . 78

A.1 An optimal path formation through a matrix of local distance measures 95 


\section{LIST OF TABLES}

2.1 Number of inputs for k-fold cross validation . . . . . . . . . . . . . . 18

2.2 Classification accuracy for individual features . . . . . . . . . . . . 19

2.3 CSEL implementation with different loading condition load . . . . . . . 21

2.4 CSEL Performance . . . . . . . . . . . . . . . . 21

2.5 Performance Comparison . . . . . . . . . . . . . . . 22

2.6 TPR and FPR Results using proposed Approach . . . . . . . . . . . . . 30

2.7 TPR and FPR Results using proposed Approach . . . . . . . . . . . . 32

2.8 TPR and FPR Results using proposed Approach . . . . . . . . . . . . . 34

2.9 TPR and FPR Results using proposed Approach . . . . . . . . . . . . . 35

2.10 TPR and FPR Results using proposed Approach . . . . . . . . . . . . 38

2.11 TPR and FPR Results using proposed Approach . . . . . . . . . . . . 39

3.1 Allocation of non-generator buses . . . . . . . . . . . 52

3.2 Active and reactive power balances in each island using proposed ap-

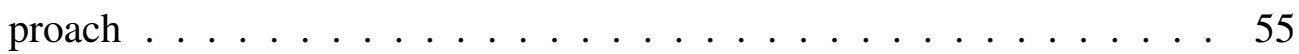

3.3 Active and reactive power balances in each island using correlationbased algorithm . . . . . . . . . . . . . . 56

3.4 Performance comparison between proposal and correlation-based algo-

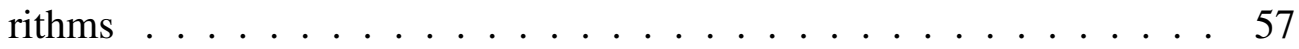

3.5 Allocation of non-generator buses . . . . . . . . . . . 58

3.6 Active and reactive power balances in each island using proposed approach ........................... 61

3.7 Active and reactive power balances in each island using community detection based algorithm . . . . . . . . . . . . . . 61

3.8 Performance comparison between proposal and community detection

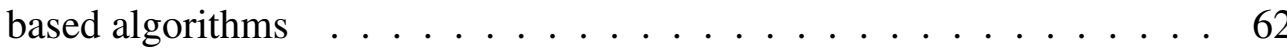


3.9 Performance comparison between proposal and community detection

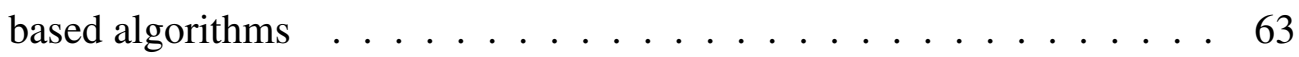

4.1 Allocation of Generators into Coherent Groups . . . . . . . . . . . . . 74

4.2 Comparison of proposed characteristic measures based coherency method for Western interconnection power system of North America . . . . . . 76 


\section{CHAPTER 1}

\section{Introduction}

With increasing energy demand, more energy resources are continuously being added in the existing network. This complex network thus operates comparatively closer to its stability limits with minimal flexibility and reliability. Such conditions may lead to low amplitude oscillations causing power fluctuations and discontinuity of supply in some cases. A major cause of wide-spread power network blackouts is an unstable system due to poorly damped electromechanical oscillations as shown in Fig. 1.1 [4]. The oscillations may exist between machines operating in a plant (inter-machine) or between the plants in distant areas (inter-area) [5]. These are called small signal stability problems. Small signal oscillatory stability is related to these low frequency small magnitude electromechanical oscillations that can be mainly categorized as: 1) oscillatory mode, 2) non-oscillatory mode. The oscillatory mode is due to lack of sufficient damping torque which is caused by the absence or non-optimal tuning of power system stabilizer (PSS). The non-oscillatory or aperiodic mode is due to lack of sufficient synchronizing torque and is dependent on automatic voltage regulator (AVR) [6]. The frequencies of these modes are typically in the range of $0.5-4 \mathrm{~Hz}$ [7]. The damping of these electromechanical oscillations needs some supplementary controls which provide controlling feedback to Automatic Voltage Regulator (AVR) of synchronous machines. Power System Stabilizer is one of the initial controllers developed to supply supplementary stabilizing signals to the excitation system of the synchronous machines [8]. They produce an electrical damping torque in phase with rotor speed deviation which is fed to generator's rotor to compensate the oscillations produced as a result of a disturbance or high power transfer through weak tie line [9], [10], [11].

These low-frequency electromechanical oscillations can be mainly classified into two categories; weakly damped or free oscillations and forced or sustained oscillations. In addition, methods to damp and mitigate free oscillations are different than those for sustained or forced oscillations. Weakly damped oscillations arise due to weak damping from fast exciters, long transmission lines, or high transmission powers [12], [13]. Weakly damped oscillation are actually a property of the system.. These oscillations are classified into local mode and inter-area mode of oscillations. Local mode of oscillations are caused by automatic voltage regulators (AVRs) operating closer to its 


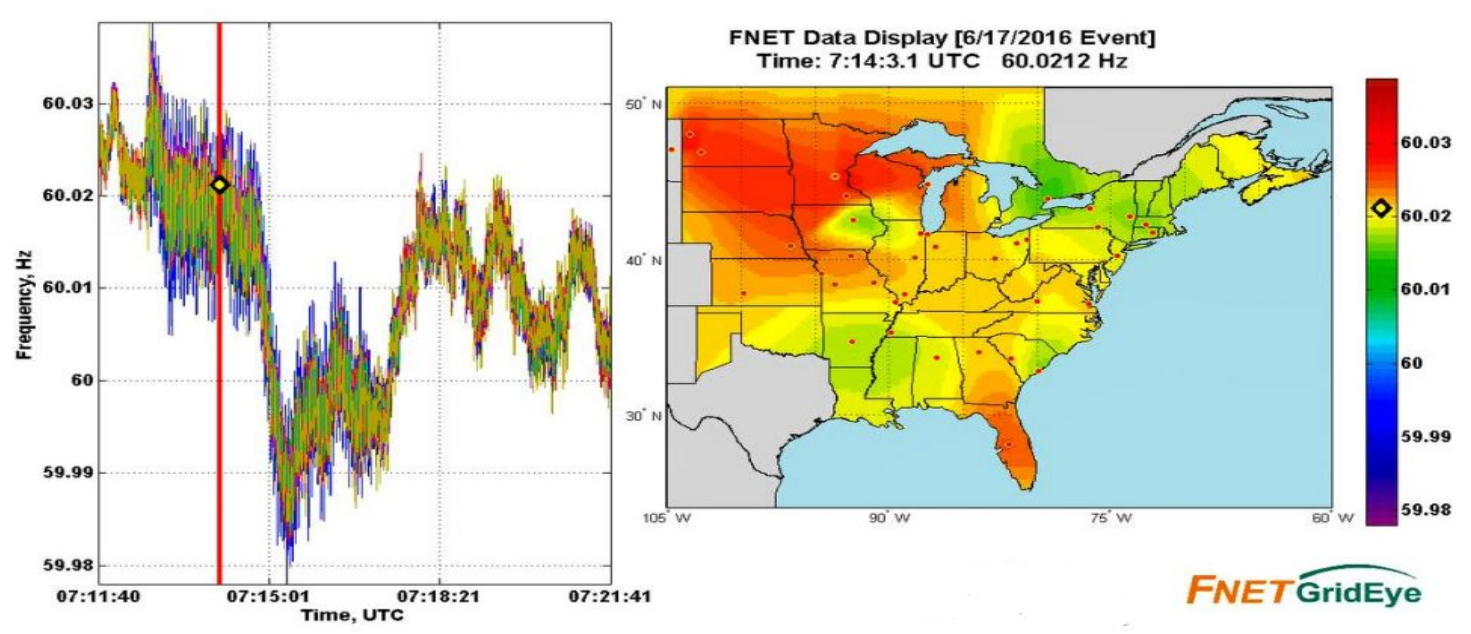

Fig. 1.1 Wide-Area forced oscillations resulting in variations of the system frequency [1]

maximum output and feeding into a weak power transmission network. On the other hand, inter-area mode of oscillations are usually caused by the weak power transmission links and heavy power transfers. Power system stabilizer (PSS) is commonly used to enhance damping and suppress these oscillations. Further, generators' power reduction and inter-link power transfer control are also useful to quench such oscillations. In weakly damped oscillations, all eigenvalues lie in the left half of the complex plane, whereas the eigenvalue having minimum absolute value lies very close to the imaginary axis. Forced or sustained oscillations are caused by cyclic loads and control loops in power plants etc. [14], [15]. This type of oscillation is not due to the general dynamics of power system, rather, it is caused by an external persistent force with a distinct oscillatory behavior. These forced oscillations can lead to voltage flickering if the oscillation frequency is around $10 \mathrm{~Hz}$, where the human eye is more sensitive. Resonance occurs if oscillation frequency approaches the system's natural mode frequency and then a small disturbance is amplified and expanded in the whole system. The most effective corrective measure against forced oscillation is to locate the source of disturbance and eradicate it from the system.

For system operators (SOs), the detection and real time analysis of these oscillations is inevitable. Therefore, recently, the attention of SOs and utilities has shifted towards tools and methods, which help in locating the source of these electromechanical oscillations [8]. In our previous works, we first investigated the impact of wind energy integration on these electromechanical oscillations [9] and then proposed a fuzzy logic based controller for damping of these oscillations [10]. However, for fast and correct remedial action, source identification of such oscillations is the foremost crucial step. For which observability and availability of measurements at different levels and diverse locations in the complex system is critical. 
In model based approaches, where availability of system parameters is not an issue, modal analysis is performed by linearizing the system in the form of state space equations [16]. Such dynamical system is expressed as a set of $n$ nonlinear differential and algebraic equations (DAE). Continuous function $f$ in $\dot{x}=f(x, z)$ represents dynamics of generators, loads as well as their associated controls; and continuous function $g$ in $0=g(x, z, u)$ describes the electrical transmission system and passive devices' behavior. Vectors $x \in R^{n_{x}}$ and $z \in R^{n_{z}}$ are the corresponding differential $(\delta, \omega)$ and algebraic variables $(V, \theta)$ respectively, and $u$ is the vector describing disturbance behavior in real world power system. The disturbance can be due to load variations, renewable energy power injections, measurement errors of control devices etc. [17]-[18]. Differential and algebraic equations of the dynamical system can be linearized into state space form as represented in (1.1). Matrices $\mathbf{A}$ and $\mathbf{B}$ are properties of the system and determined by the system structure and elements. Perturbed models of differential and algebraic variables are described as in (1.2) and (1.3) respectively.

$$
\begin{gathered}
{[\Delta x]=[A][\Delta x]+[B][\Delta z]} \\
{[\Delta x]=[\Delta \delta, \Delta \omega]^{T}} \\
{[\Delta z]=[\Delta V, \Delta \theta]^{T}}
\end{gathered}
$$

Researchers proposed several methods for oscillation source location. It was discussed by Sarmadi et al. [19] through an event in Western American power system, where a forced oscillation of $0.38-\mathrm{Hz}$, was observed. The authors also investigated the possibility of resonance between forced oscillations and electromechanical inter-area oscillations. Further, they observed that power oscillations in tie-lines may be as high as 477 MW with 10 MW forced oscillations in Kundur's two area test system due to resonance. An energy flow based method was investigated in [20] to locate the source of oscillations. The amplitude of this energy flow was equivalent to oscillation amplitude and the energy producing component with negative contribution to damping. Jin et al. [21] proposed a novel method based on search area definition through measurements to locate the source of disturbance generating oscillations. Li et al. [22] presents a robust oscillation source detection algorithm using least squares-estimation of signal parameters and damping torque analysis (DTA). The dominant mode of oscillations in each generator was extracted using measured signals (e.g. rotor angle, active power and rotor speed) by means of rotational invariance approaches like total least square-estimation of signal parameters via rotational invariance techniques (TLS-ESPRIT). The corresponding damping torque was obtained with the help of least square fitting which determines the source of oscillation. The reliability of this method, however, may be compromised if 
rotor angle and rotor speed are unavailable as well as when there are forced oscillations. Yi et al. [23] discusses forced oscillations presenting electric torque coefficient (ETC) based method. Moreover, propagation direction of disturbance may also help in locating the source of oscillations. However, it may fail for non-sinusoidal oscillations. In literature, some other methods were also presented [24] but they involve solution of high order nonlinear differential algebraic equations (DAEs). This makes them relatively computationally inefficient especially for online monitoring and decision-making. In addition, model based methods are designed for a particular operating point and with consideration of the full knowledge of the network model parameters. Thus, the performance of these methods highly depends on the reliability of system's parameters [25]. In addition, practically the operating conditions change continuously and hence the designed control gains might not be optimal in such a practically changing environment. Such difficulties hinder the model-based methods to obtain optimal solutions unless they are adaptive to the changes.

On the other hand, data driven methods use measurements collected from phasor measurement units (PMUs) to find optimal and adaptive solutions without assuming the full knowledge of the system parameters and without relying only on the current operating conditions. Moreover, recent advancements in monitoring and communication networks has dramatically increased the variety, velocity and volume of measurement data in transmission and distribution grid. Data driven analytic provides effective solutions with high performance and low computational complexity for challenging problems in power system including but not limited to, fault detection, stability analysis, load forecasting, cascading failure prediction and power system visualization. Artificial neural networks (NN), k-means clustering, support vector machine (SVM) and decision tree are some of the most popular learning techniques in data mining which are frequently utilized in power system applicaitons. For instance, revolution in power system visualization has only been possible due to useful information extraction data mining techniques. Better and interactive visualization of the power system data equips the system operator with plenty of options to implement during catastrophic events. Traditionally, system's information was available to the system operators in the form of a tabular list [26]. An example of a data-driven visualization is the contours of the per unit voltage magnitudes at $115 / 138 \mathrm{kV}$ buses in Ohio for the simulated August 14, 2003 conditions immediately before the events that led to the blackout. They provide an overview of the voltage profile of the entire region. The display is very effective for providing situation awareness. Data mining finds another interesting application in the field of load modeling and prediction which is an important aspect of electricity market. Utilities and electricity suppliers can get benefits by having the knowledge of load demand ahead of time [27]. For instance, decision tree classification and k-means clustering is employed in [28] to learn a load pattern and predict the load demand con- 
sequently. In [28], load profile of 277377 costumers over the time period of 10 months is measured. This training data trains a load behaviour classification model, which, assigns customers into different classes based on their load behaviour. Eventually, load demand forecasting is performed according to the class assigned to each customer [26]. Further, event Detection utilizing data driven methods is one of the most studied application in power system. When the generated power exceeds the load demand, frequency of the system increases; whereas, an excess of power consumed decreases the frequency. K-medians clustering has been utilized in [29] to detect disturbance events using frequency measurements. Data samples are divided into two clusters as pre-event and post-event clusters. The severity of the event is determined through the difference between the centroids of the two clusters. K-medians is less sensitive to outliers as compare to k-means. Hence, it is more effective for this task since the frequency data usually has large amount of outliers [26]. To ensure stability, voltage at various locations in the system is continuously monitored. By analyzing the type and severity of the voltage disturbances through effective data extraction and mining techniques, useful information about the state of the whole inter-connected system and potential faults can be extracted. Support vector machine has been utilized in [30] to classify voltage disturbances. Features like three phase rms voltages, total harmonic distributions and third harmonic magnitudes were used to classify the voltage disturbances. Five classes termed as: Single-phase-to-ground fault, three phase fault, phaseto-phase fault, transformer energizing and double-phase-to-ground fault were assigned for voltage disturbances. Support vector machine identifies the fault type in the system and hence system operator takes actions accordingly [26]. Hence, recent researches showed increasing interest in utilizing these learning techniques for stability, islanding detection and cyber-attacks detection as well. SVM was successfully applied for post fault instability prediction using PMU data in [31]. It can also be used to predict the voltage instability and fault power swing [32]. Recently few more data mining based intrusion detection and malicious attack detection approaches were also studied [33]. Data mining techniques such as support vector machine (SVM), random forest classifier, extreme learning machine (ELM) etc. may provide alternate computationally efficient solution which is needed for oscillation source detection phenomenon [34], [35]. There is not much literature for computationally efficient data mining techniques that have been applied for detecting oscillation source in power system.

Distribution systems within the power grids are dynamic with load and generation varying and a major disturbance can jeopardize and endanger the operation and security. These distribution networks are even more critical and are very preeminent now due to newer additions such as electric vehicles, renewable sources and active end users [36]. As penetration of these additions increases in microgrids, a more dynamic and complex system will emerge making real-time monitoring and control rather challenging due to 


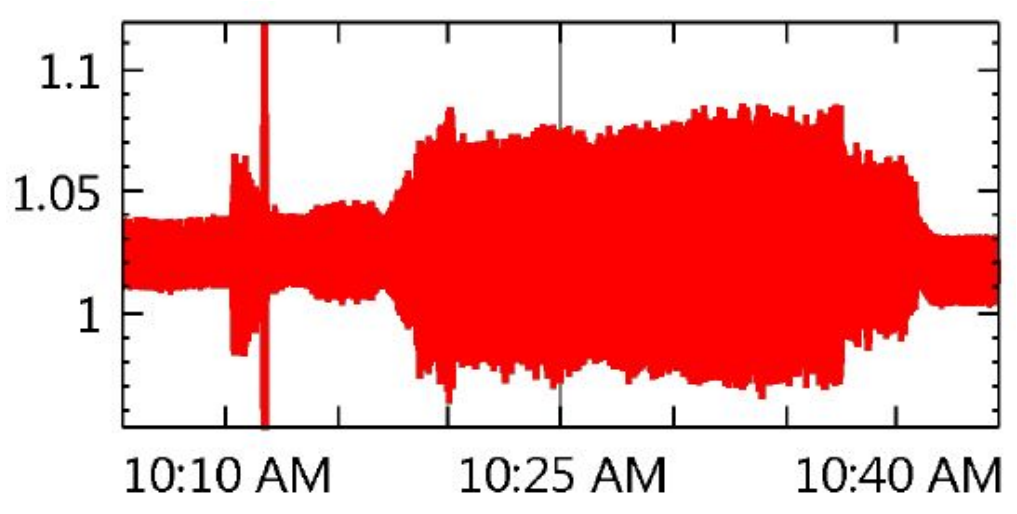

Fig. 1.2 Voltage variations during $3 \mathrm{~Hz}$ oscillation event at wind power plant [2]

stochastic dynamic encumbrances.

In a microgrid lack of spinning reserves and low physical inertia result in faster dynamics than conventional rotating machines [37]. It brings up new challenges in the microgrid control area which needs to be addressed and solved. One of such challenges is its high susceptibility to oscillations resulting from highly fluctuating loads, intermittent renewable sources or malfunctioning of feedback controllers [38, 15, 14]. For instance, in November 2016, an oscillation event was observed in the AEP (American Electric Power) network while monitoring a solar power plant [2]. The oscillations in voltage persist over multiple days. It is to be noted that these oscillations occurred in the middle of the day especially during periods of high irradiance. Moreover, in December 2012, a line outage triggered oscillations at a $60 \mathrm{MW}$ wind farm interconnected at 69 $\mathrm{kV}$ [2]. Variations in the voltage magnitude were as high as 18 percent at a frequency of $3 \mathrm{~Hz}$ as shown in Fig. 1.2. The event adversely affected the operation and took half hour restoring the system to normal operation. Hence, the operation of the complex network with the increased penetration of these intermittent resources is very challenging for the system operators. These oscillations may cause increased losses, power quality degradation, increased EMI and converter overloading [39].

One method to mitigate these oscillations is to design a controller to provide adequate damping. However, it is not a robust solution as its performance is highly sensitive and dependent on the system parameters and structure, which may vary in a microgrid due to its nature and expansion. Researchers suggested that designing controls to damp these oscillations is impractical [40]-[41]. Instead, disconnecting the source and subsequent investigation of the causes of the disturbance is the main solution. A recent literature survey [42] outlined a variety of source identification methods including the main requirements of these methods. A library of test cases for validating source identification methods is presented in [43]. Hybrid techniques, which leverage both a system model and measured data were demonstrated in [44] and [45]. These techniques use measured signals as inputs for system model and after simulating the model, the out- 
puts are compared with their corresponding measured signals. Significant divergence between the model outputs and measurements indicates the presence of forced oscillation.

A promising method, which has shown its practical performance in over 50 actual events from WECC and ISONE, is the Transient Energy Flow (TEF) method [12]. It tracks the net transient energy flow in all lines where measurement data is available, thus is inherently model independent. While carrying the advantage of being model free, it has few shortcomings, the most important one is that it can not distinguish between a true oscillation source bus and a bus with net "negative damping" contribution, since both are seen as sources of Transient Energy. In addition, the network is assumed to be lossless, which is very crucial for this method [41]. A comprehensive discussion on the open questions about this method can be found in [46]. Some other methods have also been presented [47], [48] but they involve solutions of high order nonlinear differential algebraic equations (DAEs). This makes them relatively computationally inefficient especially for online monitoring and decision-making. Moreover, these methods were developed and tested in transmission system, where PMU measurements at almost each bus are readily available. In addition, the presence of inertia in the form of rotating synchronous machines is also extremely helpful against these oscillations. On the other hand, with increased penetration of DERs, there is a pressing need to analyze these oscillations in islanded microgrid which is not connected or supported through large interconnected transmission system. The proximity of these DERs in a small geographical area may also generate the possibility of having more than one sources of oscillation.

It is clear that there is a need in the power systems community for the development of a more systematic approach which do not heavily rely on strong model assumptions, considers the possibility of more than one sources and more importantly analyze and identify the cause of these oscillation in an islanded microgrid.

In situations where oscillation source detection and damping technologies fail to perform, the system may experience a cascading outage scenario as shown in Fig. 1.3, where two cascading outages occurred at $\mathrm{t}=5 \mathrm{sec}$ and $\mathrm{t}=7 \mathrm{sec}$, and one generating unit lost synchronism. Hence, the system eventually became unstable at $t=11.45 \mathrm{sec}$. An efficient solution to avoid the instability is the adaptive islanding which should separate generating units with different dynamic behavior and ensure that coherent generating units remain in the same island: 1) to improve the transient stability; and 2) to reduce the chances of further outages. The key step for adaptive islanding is to find the coherency among generating units.

In summation, this work makes the following contributions to the online grid stability monitoring and providing adaptive emergency response.

- Chapter 2 development of an explicit algorithm that can use streaming real-time data from the measuring units to detect the source of oscillations, which may 


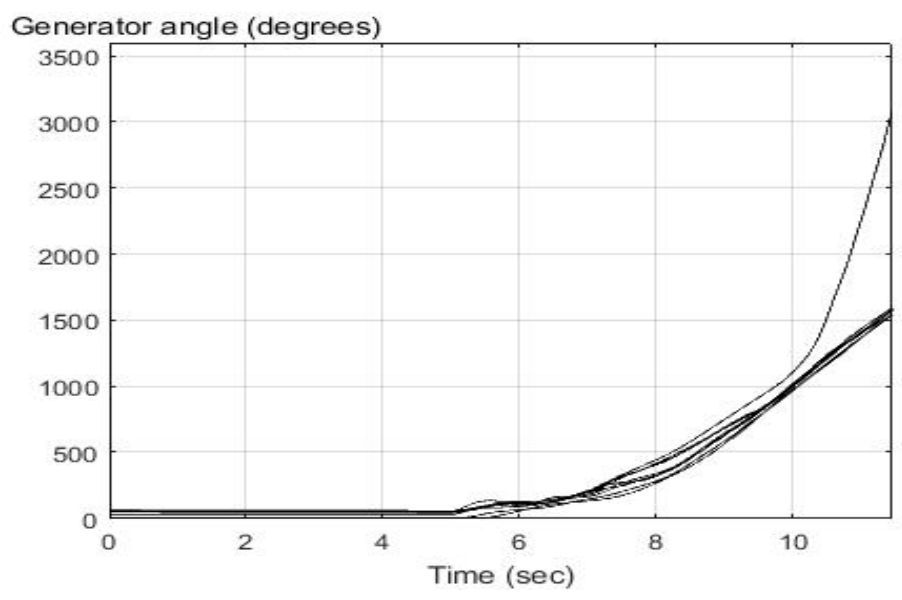

Fig. 1.3 Generator response following cascaded outages

further cause instability if not properly detected and mitigated. Applicability of the proposed approach to real-time topology change scenarios is also analyzed. In addition, it investigates power oscillations in islanded microgrid and demonstrate the experimental results finding their source of disturbance. Scenarios with more than one sources causing these oscillations are also considered. Applicability of the proposed approach on oscillation cases caused from highly fluctuating loads and performance in the presence of ambient noise are also checked.

- Chapter 3 presents an adaptive generators coherency identification method based on dynamic time warping. It validates its better performance against benchmark methods through intentional islanding in case of cascading failures. A unique feature of dynamic time warping is its applicability during partial observability, is also discussed.

- Chapter 4 investigates structural characteristic measures which serve as an effective cumulative coherency identification metric using time series data from phasor measurement units.

- Chapter 5 explains future work that can be leveraged and carried out from this work 


\section{CHAPTER 2}

\section{Oscillation Source Identification Using Data Driven Credibility Search Ensemble Learning}

\subsection{Introduction}

Power grids are large cyber-physical systems with physical operation controlled and integrated through communications. The technological breakthroughs made by the availability of low-cost, high speed communications, larger storage spaces, greater internet bandwidths have led to increased attentions towards advantages of these systems. The use of phasor measurement unit in complex interconnected power system for monitoring and control has increased significantly. This has resulted in huge amounts of data and growing databases, thus gearing towards an era where utilities might encounter an enormous amount of data daily from their measurement units including sensitive information useful for daily operations.

Data mining techniques such as support vector machine (SVM), random forest classifier, extreme learning machine (ELM) etc. may provide alternate computationally efficient solution which is needed for oscillation soure detection phenomenon [34], [35]. There is not much literature for computationally efficient data mining techniques that have been applied for detecting oscillation source in power system. The proposed ensemble learning approach based on data mining can be employed to locate the source of oscillations in power system. Recent researches showed increasing interest in utilizing these learning techniques for stability, islanding detection and cyber-attacks detection as well. SVM was successfully applied for post fault instability prediction using PMU data in [31]. It can also be used to predict the voltage instability and fault power swing [32]. Recently few more data mining based intrusion detection and malicious attack detection approaches were also studied [33].

This chapter presents an ensemble learning based approach to locate the source of electro-mechanical oscillations in complex power system. As compared to existing methods, contributions of this work are in the:

1. Development of a data driven methodology that can use streaming real time data from the measuring units deployed across the system to detect the source of oscil- 
lations, which may further cause instability if not properly detected and damped.

2. Applicability of the proposed approach to real time topology change scenarios

3. Robust performance in the presence of measurement noise.

\subsection{Classification Models for Oscillation Source Location}

Consider measurement vector $X=\left\{X_{1}, X_{2}, X_{3}, \ldots, X_{N}\right\}$ with $X_{i}=\{V, \theta, \delta, \omega\}$ and associated oscillation source location vector $Y=\left\{y_{1}, y_{2}, y_{3}, \ldots, y_{M}\right\}$; where $N$ number of PMUs are monitoring the power system with each reporting $M$ features [49]. The resulting measurement set D, for training stage, having PMU measurements and corresponding source location information can be formed as in (2.1). In this work, we considered $M=4$ and $N=179$.

$$
D=\left[\begin{array}{ccccc}
x_{11} & x_{12} & \cdots & x_{1 N} & y_{1} \\
x_{21} & x_{22} & \cdots & x_{2 N} & y_{2} \\
\vdots & \vdots & \ddots & \vdots & \vdots \\
x_{M 1} & x_{M 2} & \cdots & x_{M N} & y_{M}
\end{array}\right]
$$

A non-linear mapping $k\left(x_{M N}, z_{p q}\right)$ transforms original measurements $x_{M N}$ into high dimensional space measurements $z_{p q}$ to form the classification model of Support Vector Machine (SVM). Decision function $f(x)=\operatorname{sign}\left\{\sum_{j=1}^{P^{S V}} a_{j} y_{j}^{S V}\left(\Phi(P) . \Phi\left(P_{j}^{S V}\right)\right)+\right.$ b\} distinguishes transformed measurements $z_{p q}$ by defining optimal separable hyperplanes $P_{j}^{S V}$. Here, $\Phi(P) . \Phi\left(P_{j}^{S V}\right)$ is the kernel function that maps original low dimensional measurements $x_{M N}$ into higher dimensional feature space and $y_{j}^{S V}$ is the oscillation source location corresponding to $j$ th hyperplane or support vector [50]. Also $a_{j}$ is the Lagrangian multiplier and $b$ is the bias obtained by minimizing the following objective function:

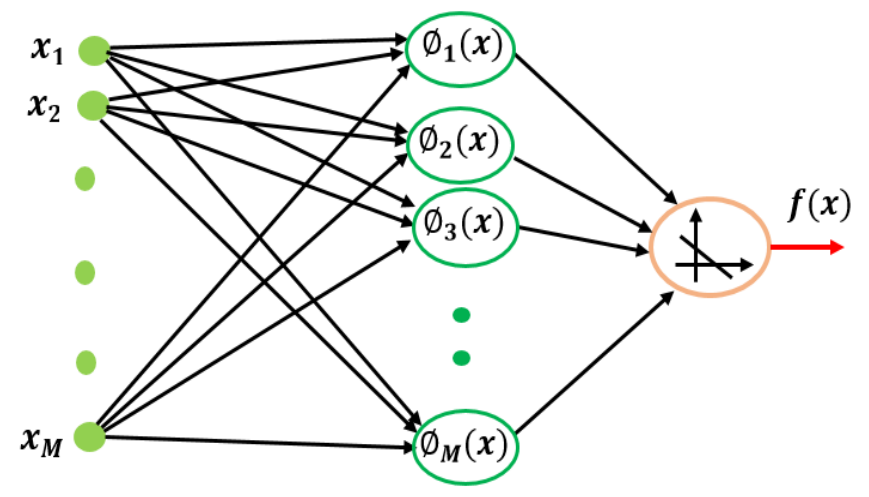

Fig. 2.1 Mapping of input vector to higher dimensional feature space using kernel function 


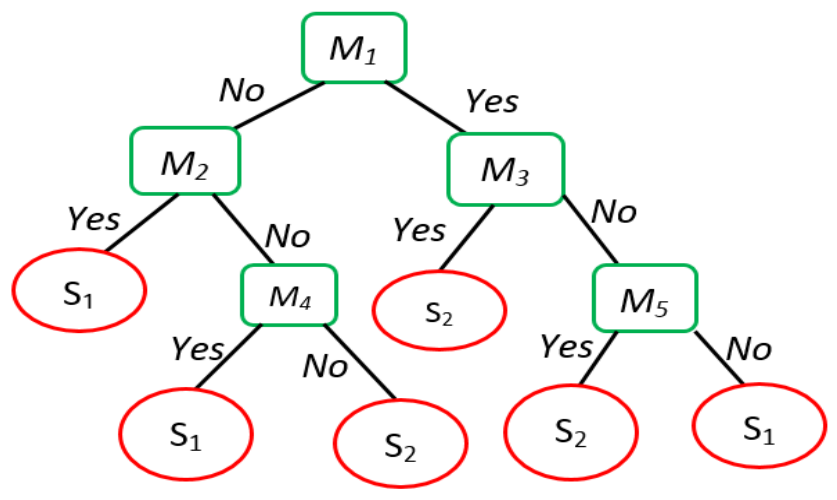

Fig. 2.2 Learning process from available measurements to the desired source location

s.t $\gamma_{i} \geq 0 \quad \forall i=1, \ldots, N$

$$
\min _{w, b, \gamma} \frac{1}{2}\|w\|^{2}+C . \sum_{i=1}^{N} \gamma_{i}
$$

$$
y_{i}\left(w \cdot x_{i}+b\right) \geq 1-\gamma_{i} \quad \forall i=1, \ldots, N
$$

where, $\gamma$ is the distance from separable hyperplane for each misclassified measurement and $C$ is the associated penalty factor, during offline training stage. Further, $w$ is the perpendicular vector to separating hyperplanes that when minimized in turn maximizes the margin of separability in transformed high dimensional measurements $z_{p q}$. Fig. 2.1 maps feature measurements $x_{1}, x_{2}, \ldots, x_{M}$ into higher dimensional space $\phi_{1}(x), \phi_{2}(x), \ldots, \phi_{M}(x)$; where each feature measurement $x_{i}$ is transformed into multidimensional measurement space $\phi_{j}(x), i<j$. It is to be noted that such multidimensional mapping helps in finding a linear or non-linear decision boundary in the transformed space while maximizing the margin of separability between source location labels. The structure of the decision boundary depends on the type of the kernel function.

The second classification model considered in this work is Decision Tree (DT), which uses multiple thresholds, unlike separable hyperplanes, to simplify the complex decision process into a collection of simple decision boundaries. For instance as shown in Fig. 2.2, the learning process initiates at each internal node where feature measurement $M_{i}$ results into two sub-branches depending on he outcome of a decision rule [49]. This learning process recursively splits measurement set into two subsets, following the decision rule, and eventually resulting in a terminal node $S_{i}$ that represents the corresponding source of oscillation. During online stage, a path can be traced from internal node $M_{i}$ to the terminal node $S_{i}$ using real time feature measurements and thus the source of oscillations can be located.

The third classification model termed as Random Forest (RF) considers a forest or multiple of decision trees for source localization, in addition to building a single tree. However, the final oscillation source location is determined by the mode of de- 
cisions from each decision tree. For instance, assume a feature vector $\left(x_{i}, y_{j}\right), i, j \in$ $\{1,2,3, \ldots, M\}$ from which training sets $\mathrm{R}$ are drawn randomly. The goal is to build a forest $h$ of decision trees $\left\{d_{1}, d_{2}, d_{3}, \ldots, d_{R}\right\}$, which can predict oscillation source location $y_{j}$ from feature measurements $x_{i}$ and is trained using $\mathrm{R}$ training sets. Each training set $d_{i}$ is sampled with replacement from feature measurement set $x_{i}$. Each $d_{i}$ is a bootstrap sample of $x_{i}$ so that some tuples may occur more than once in $d_{i}$, while others may be excluded [51].

\subsection{Overview of Proposed Approach}

Utilizing the success in other fields [32],[33], an ensemble learning approach based on novel credibility search is proposed in this work for oscillation source location problem. Consider $O$ classes, each representing a unique location of oscillation source, denoted by $\left\{l_{1}, l_{2} \ldots, l_{O}\right\}$, and $K$ classification models denoted by $\left\{Y_{1}, Y_{2} \ldots, Y_{K}\right\}$. A classifier $Y_{k}$ may cover all classes $O$ as general classification model or a small subset $\left\{l_{i}\right\}_{i \in I_{k}}$ as specialized classification model, where $I_{k}$ is the set of location indices.

Classification model $Y_{k}$ yields a prediction vector $p_{k} \in R^{\left|I_{k}\right|}$ with $\left|I_{k}\right|$ dimensions, for a given sample set $x$. Each element $p_{k}(i)$ with $i \in I_{k}$, in such prediction vector $p_{k}$, accounts for the probability of sample set $x$ with source location $l_{i}$ from the perspective of classifying model $Y_{k}$. From this location prediction $l_{i},\left|I_{k}\right|-1$ location preferences can also be derived and given by $\left\{l_{j} \rightarrow l_{i}: \forall j \in I_{k} n\{i\}\right\}$, signifying that the classification model $Y_{k}$ prefers $l_{i}$ over other potential oscillation source locations in its domain $I_{k}$.

When all classification models $\left\{Y_{1}, \ldots, Y_{K}\right\}$ exhibit consistent preferences with each other, it is not challenging to reach an overall prediction by tracing these preferences. However, often real world problems are more intricate and individual classification models may predict contradicting locations for source of oscillation. For example, in Fig. 2.3, majority voting yields an overall wrong prediction even though individual classification models are predicting correctly. There are four locations of oscillation $\left\{l_{1}, l_{2}, l_{3}, l_{4}\right\}$ and three classification models $\left\{Y_{1}, Y_{2}, Y_{3}\right\}$ each covering a pair of locations. Dashed lines show critical boundaries for each classification model. If a sample $x_{i}$ is from location $l_{4}$, classification models $Y_{1}$ and $Y_{2}$ classify it as location $l_{3}$ with only $Y_{3}$ classifying as location $l_{4}$. Since location $l_{4}$ gets one vote whereas location $l_{3}$ gets two votes, directly combining votes from classification models would result in fallacious prediction $l_{3}$. Hence, in this work to combine the decisions of classification models, we rely on the equilibrium distribution, explained in the following section, to predict the location of oscillation source.

In credibility search approach, an ensemble of classification models $\left\{Y_{1}, Y_{2}, Y_{3}\right\}$ whose individual decisions are combined by weighted approach, locates the source of 


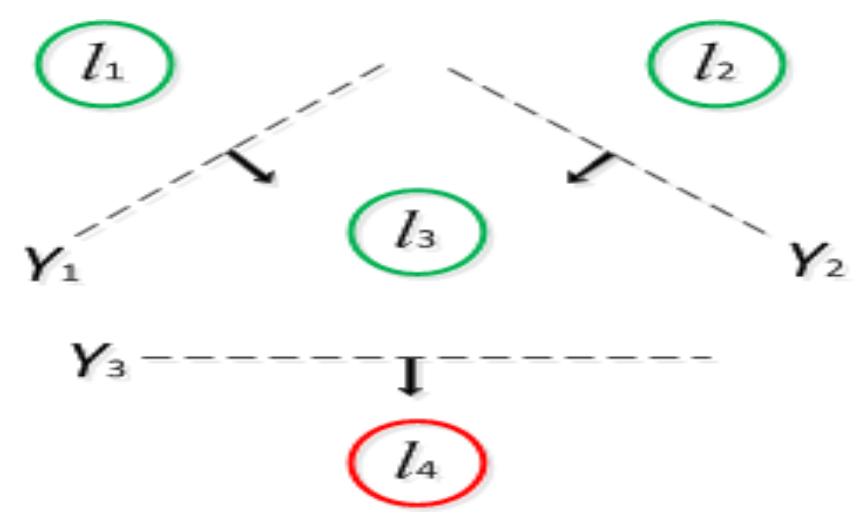

Fig. 2.3 Majority voting leading towards erroneous prediction

oscillation using online measurements. The final decision $\pi=\left\{Y_{1}, Y_{2}, Y_{3}\right\}$, in the form of possible location of oscillation source, is aggregated by weighted decisions from each classification model. The weight of these classification models is determined during offline credibility search. In the example shown in Fig. 2.3, classification model $Y_{3}$ gets the highest weight for location $l_{4}$ through the proposed credibility search approach.

\subsubsection{Proposed Credibility search based Oscillation Source Identification}

Consider a sample data set $x$ and its associated prediction vector $p_{k}$ derived from classification model $Y_{k}$. For class $l_{i}$ and $l_{j}$, where $i, j \in I_{k}, i \neq j$, we can write a transition matrix $\mathrm{Q}$ using the prediction values $p_{k}(i)$ and $p_{k}(j)$ as [52]:

$$
Q=\left[\begin{array}{cc}
-p_{k}(j) & p_{k}(j) \\
p_{k}(i) & -p_{k}(i)
\end{array}\right]
$$

Here, $\left(p_{k}(i), p_{k}(j)\right)$ reflects the relative preferences of classification model $Y_{k}$ for location classes $l_{i}$ and $l_{j}$. An equilibrium distribution $\pi$, reflects which location class $l_{i} \in\left\{l_{1}, l_{2}, \ldots, l_{O}\right\}$, the classification model $Y_{k}$ is likely to reach in the long run and it is related to the transition matrix $\mathrm{Q}$ by a stationary condition as $\pi^{T} Q=0$. Once transition matrix $\mathrm{Q}$ is constructed from prediction vector $p_{k}$, equilibrium distribution $\pi$ can be solved using linear algebra. Transition matrix $\mathrm{Q}$ from class $i$ to $j$ can be written as:

$$
Q(i, j)=q_{i j} \cong \begin{cases}\sum_{k \in S_{i j}} p_{k}(j) & (i \neq j) \\ \left.-\sum_{j^{\prime} \neq i} q_{(} i j^{\prime}\right) & (i=j)\end{cases}
$$

where, $S_{i j}=\left\{k: i \in I_{k}\right.$ and $\left.j \in I_{k}\right\}$ is the index set of classification models that cover both location classes $l_{i}$ and $l_{j}$. Equilibrium distribution $\pi$ can be solved as the required final location prediction, once transition matrix $\mathrm{Q}$ is constructed.

Proposition 1. For a transition matrix $\mathrm{Q}$, the overall prediction $\pi$ is

$$
\pi(i)=\frac{1}{\left|Y_{i}\right|} \sum_{k \in Y_{i}} w_{k} p_{k}(i)
$$


with $w_{k}=\sum_{j \in I_{k}} \pi(j)$ and $Y_{i}$ is the set of classifiers who cover the source location class $l_{i}$.

Proof. Let $a_{k i}=I\left(i \in I_{k}\right)$, then $q_{i j}$ may be written as:

$$
q_{i j}= \begin{cases}\sum_{k} a_{k i} a_{k j} p_{k j} & (i \neq j) \\ \sum_{k} a_{k i} a_{k j}\left(p_{k}(j)-1\right) & (i=j)\end{cases}
$$

when $i=j$ then we can write $a_{k i} a_{k j}=a_{k i}$. Hence $\pi^{T} Q$ can be expanded into

$$
\pi^{T} Q(j)=\sum_{i} \sum_{k} a_{k i} a_{k j} p_{k j} \pi(i)-\sum_{k} a_{k j} \pi(j)
$$

with $\pi^{T} Q=0$, we obtain

$$
\begin{gathered}
\pi(j)=\left(\sum_{k} a_{k j}\right)^{-1} \sum_{i} \sum_{k} a_{k i} a_{k j} p_{k j} \pi(i) \\
\pi(j)=\frac{1}{\left|Y_{i}\right|} \sum_{k \in Y_{i}}\left(\sum_{j \in I_{k}} \pi(i)\right) p_{k}(j)
\end{gathered}
$$

swapping $i$ and $j$, we obtain

$$
\pi(i)=\frac{1}{\left|Y_{i}\right|} \sum_{k \in Y_{i}} w_{k} p_{k}(i)
$$

It indicates that $\pi(i)$ is the weighted sum of predictions $p_{k}(i)$ from all classification models $Y_{i}$ covering class $l_{i}$. The weight $w_{k}=\sum_{j \in I_{k}} \pi(j)$ can be reflected as the relevance of the classification model $Y_{i}$ for the given sample data set $x$. Prediction value $p_{k}(i)$ is the relative preference of a classification model $Y_{k}$ for a particular class label or source location $l_{i}$. For example for a sample $x$ the prediction value $p_{k}(i)$ from a classification model $Y_{k}$ is simply its preference for all possible case labels or source locations $l_{i}$. In case of dual class label problems, $p_{k}(i)$ is either 0 or 1 . However, for the multiclass source location problems, the resulting prediction $p_{k}$ is obtained from the trained classification model $Y_{k}$ and ranges between $0 \sim 1$ for any given sample $x$.

When all classification models $\left\{Y_{1}, Y_{2} \ldots, Y_{K}\right\}$ cover the same set of location classes $\left\{l_{1}, l_{2}, \ldots, l_{O}\right\}$ we have:

Corollary 1. If each classification model covers the entire possible location class space, then $\pi$ equals the average of individual predictions:

$$
\pi=\frac{1}{K} \sum_{k=1}^{K} p_{k}
$$

This corollary evolves directly from Proposition 1 . Specifically, $w_{k}=1$ for each clas- 


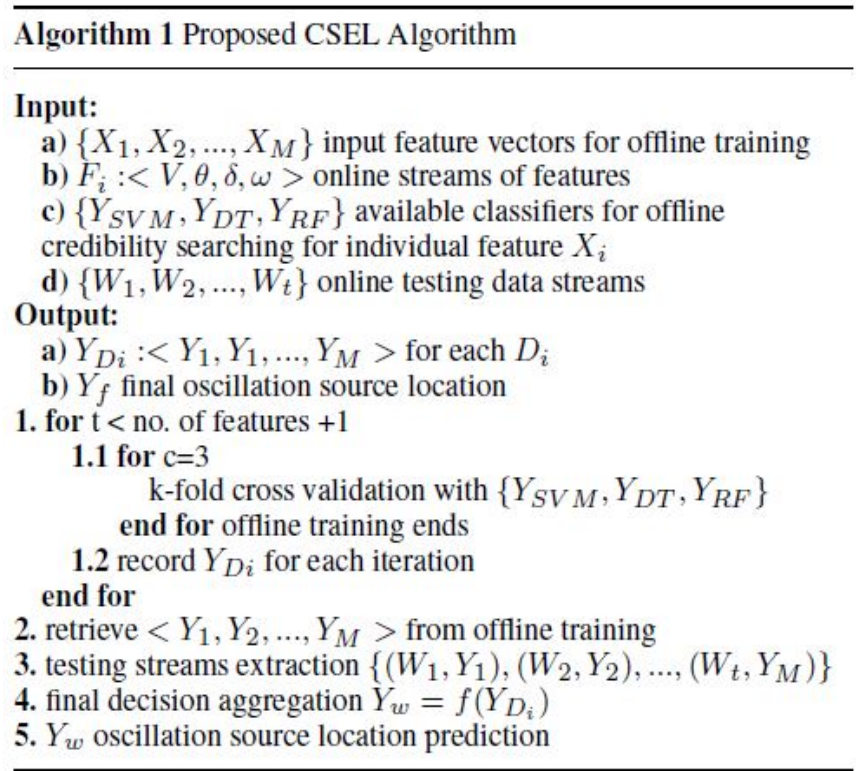

sification model $Y_{i}$ in this scenario. We further expanded this corollary by finding the credibility of each classification model $Y_{i}$ during offline validation stage and set its $w_{i}=1$ and $w_{j}=0, \forall j \in I_{k} \backslash\{i\}$ for rest of the classification models $Y_{j}$.

Individual classification models $\left\{Y_{S V M}, Y_{D T}, Y_{R F}\right\}$ are trained offline for data $\mathrm{D}=$ $\left[\left(\left\{x_{11}, x_{12}, x_{13}, \ldots, x_{1 N}\right\}, y_{1}\right),\left(\left\{x_{21}, x_{22}, x_{23}, \ldots, x_{2 N}\right\}, y_{1}\right), \ldots,\left(\left\{x_{M 1}, x_{M 2}, x_{M 3}, \ldots, x_{M N}\right\}, y_{M}\right)\right]$.

An ensemble of these classification models, whose individual decisions are combined by weighted approach explained above locates the source of oscillation using online measurements. This credibility search ensemble learning approach is explained in detail in Algorithm 1.

PMU measurement set $X=\left\{X_{1}, X_{2}, X_{3}, \ldots, X_{N}\right\}$ with feature vector $X_{i}=\{V, \theta, \delta, \omega\}$ and classification models $\left\{Y_{S V M}, Y_{D T}, Y_{R F}\right\}$ with relatively high prediction accuracy are validated using $\mathrm{k}$-fold cross validation. The classification model that has maximum accuracy, for each feature during offline validation, is selected as credible model during online source localization stage. The final decision $\pi=f\left\{Y_{1}, Y_{2}, \ldots, Y_{K}\right\}$, contains possible locations of oscillation source obtained by aggregating weighted decisions of each classification model. Fig. 2.4 sequentially describes the complete implementation of the proposed credibility search ensemble learning approach. The box on the left depicts offline validation stage; Database, containing several oscillation scenarios along with their source locations as presented in section 2.4. Different features from this database are extracted and classification models trained using these features and ranked according to their performance are obtained. Highly ranked models are utilized by source prediction module of online stage as shown by box on the right in Fig. 2.4. During online operation, similar features are extracted from real time PMU measurements and fed to the source prediction module. This critical module aggregates the final decision based on the individual predictions of highly ranked classification models and 


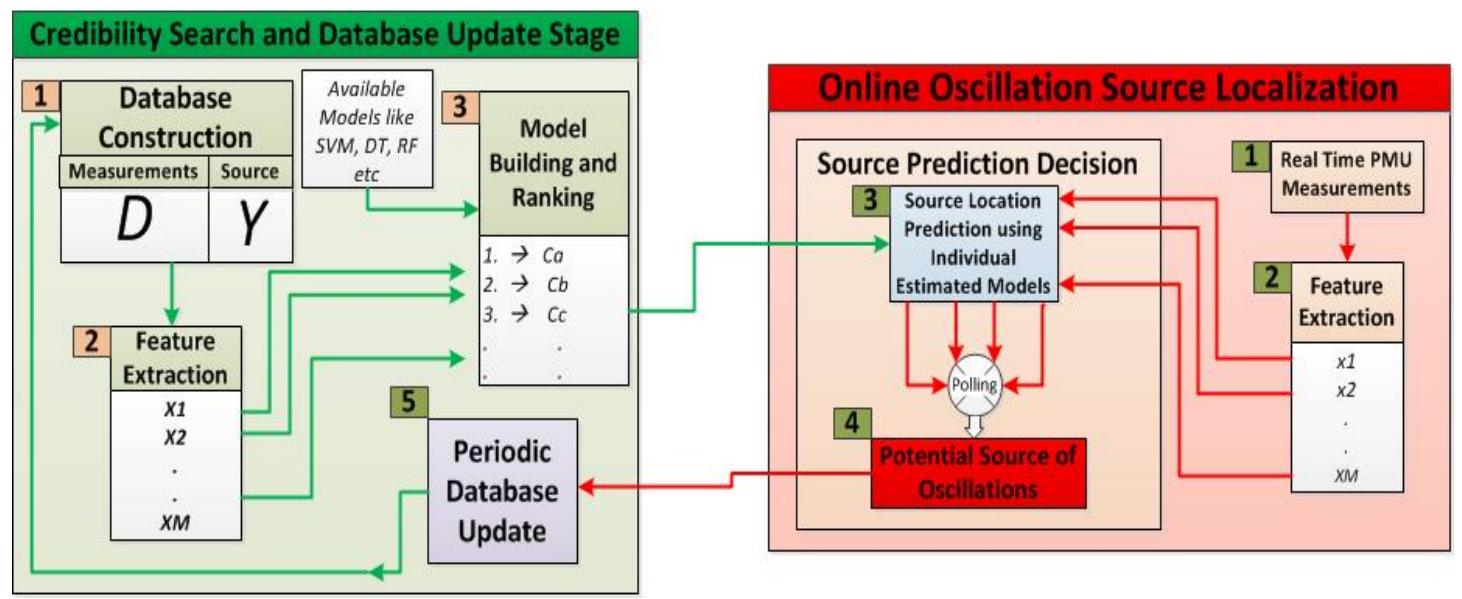

Fig. 2.4 Implementation flow of proposed credible search ensemble learning approach; green box: offline validation stage, red box: online prediction stage

feature measurements from real time PMU data.

\subsection{Performance Evaluation Indices}

During offline validation, the credibility of classification models for each individual feature is determined using accuracy and misclassification rate defined as [53]:

$$
\begin{aligned}
& \text { Classifier Accuracy }=\frac{\text { Correctly classified instances }}{\text { Total number of instances }} \\
& \text { Misclassification Rate }=\frac{\text { Misclassified instances }}{\text { Total number of instances }}
\end{aligned}
$$

\subsubsection{Measurement Error Handling}

The robustness against PMU measurement errors or missing values is another crucial criterion to check the performance of any online algorithm. In this work, cyber-attacks or data integrity attacks are not considered, hence missing values are due to communication link over-trafficking or PMU sensor faults; there may be instances where there will be no data for features. Since proposed CSEL assigns unique classification model to each individual feature during offline credibility search, there will be negligibly small or no impact of missing values. Oscillation source prediction will be dependent on available feature measurements and their corresponding models.

\subsubsection{Topology Changes Impact}

Performance of CSEL algorithm should also be tested against normal day-to-day topology changes. These topology changes are often due to maintenance and operational requirements. Therefore, an online source location approach should be at-least marginally 
pruned to these practical topology changes.

\subsection{Credibility Search of Classification Models}

\subsubsection{Test System}

To train and assess the performance of classification models for each individual feature reported by PMU, Western Interconnection of North America WECC 179-bus system was used. This system has recently been recently highly recommended by IEEE PES Task Force on Oscillation Source Location [54] to study and evaluate performance of oscillation source location approaches. It has 29 generators and 263 transmission lines.

\subsubsection{Training Data}

To select the most credible classification models for each reported feature for online source prediction, simulation data developed in [55] was partially used. This data was particularly developed to analyze oscillations source location algorithms. In this model PMUs placement guarantees the full observability of the system with reported features of: 1) bus voltage magnitude, 2) bus voltage angle, 3) generator speed, 4) rotor angle. Classical models were considered for generators for dynamic simulations and 40sec time domain simulated outputs from DSAT were utilized to construct the training data. This data include single and multiple oscillation sources along with sources that include local as well as inter area modes of oscillations. The damping coefficient D was altered and three phase to ground faults were simulated for $0.055 \mathrm{sec}$ at certain critical locations in DSAT. The source of these oscillations is not imperative as source is not always the part of system which is actually disturbed. Additionally, source of oscillations may be a generator with negative damping as shown in Fig. 2.5 [55].

A total of 21 cases were considered with 12 cases of forced oscillations. For forced oscillations, injected excitation signal frequency coincided with the frequency of the local or inter area modes. The data set was partitioned into two subsets for validation; learning and testing sets. The number of training subsets are (k-1) and there is 1 testing subset in a k-fold cross validation. Training and testing is repeated $\mathrm{k}$ times using different (k-1) subsets each time. The overall accuracy of the validation stage is computed from the average of each iteration. To search the most credible model for each reported feature, a 10-fold cross validation was performed. Further, in each subset, features were divided into two categories; 1) bus voltage magnitude and voltage angle, 2) rotor angle and generator speed. A total of 1202 instances for each $40 \mathrm{sec}$ simulation case were considered of which 21 are the number of oscillation source cases considered for offline credibility search. For each $40 \mathrm{sec}$ case, feature measurements of voltage magnitude and angle from 179 PMUs were considered for voltage magnitude and angle. This 


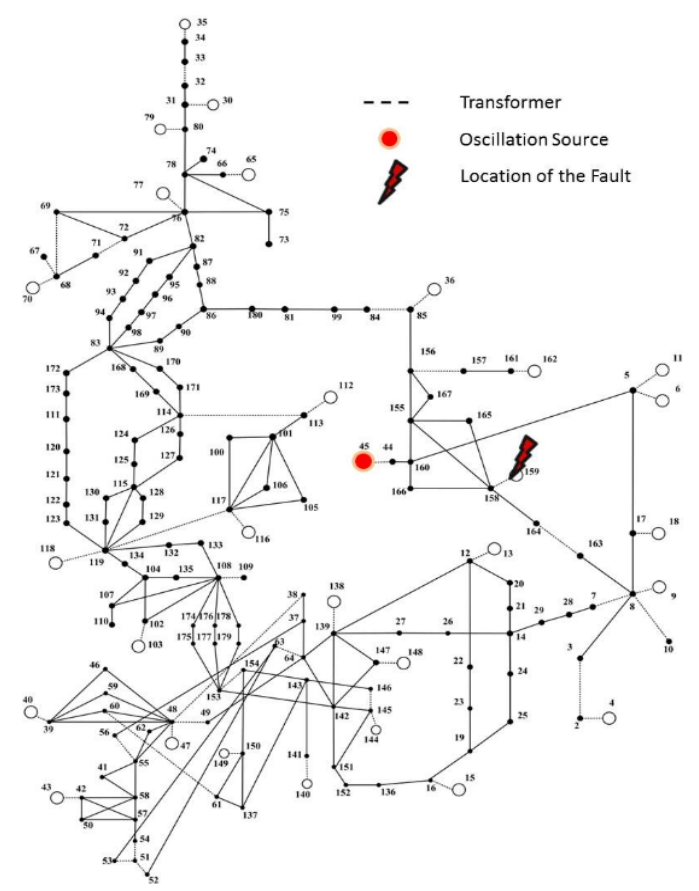

Fig. 2.5 Three phase short circuit fault at bus 159 in WECC 179-bus system

Table 2.1 Number of inputs for k-fold cross validation

\begin{tabular}{|l|l|}
\hline Set of Features & Total Number of Instances \\
\hline \hline Bus Voltage Magnitude and Angle & $179 \times 1202 \times 21=4518318 \simeq 4.5$ million \\
\hline Generator Speed and Rotor Angle & $29 \times 1202 \times 21=732018 \simeq 0.7$ million \\
\hline
\end{tabular}

resulted in 29 estimated values of rotor angle and rotor speed for the generators. The total number of instances for offline validation can be seen in Table 1 for each category. The simulation was performed on a 64 bit Intel Core i7 Central Processing Unit with 3.00 gigahertz speed, 16 giga bytes installed memory (RAM) and 1 terra byte hard disk space.

\subsubsection{Selection of most Credible Classification Models}

The selection of classification models for each reported feature is dependent on their performance during offline credibility search. Table 2.2 and Fig. 2.6 indicate oscillation source prediction accuracy for each individual measurement feature during validation and offline stage. RF performs better with voltage magnitude and generator speed as feature measurement vector with $98.31 \%$ and $97.91 \%$ accuracy respectively. On the other hand, SVM outclasses others for voltage angle measurements with $94.07 \%$ prediction accuracy. For rotor angle measurement vector, DT (J48) outperforms other models and shows $94.86 \%$ accuracy. Fig. 2.8. shows that RF has the lowest mis-classification rate as compare to others, when all features are combined. The final source location uses decisions from most credible classifier for each feature. 


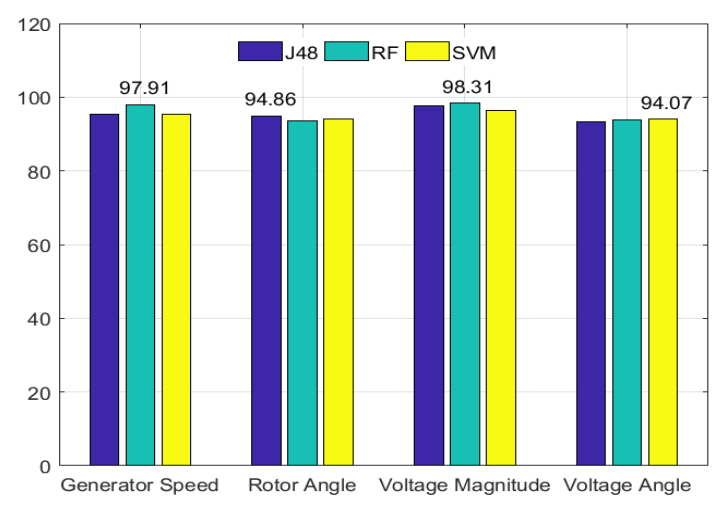

Fig. 2.6 Prediction accuracy for each individual classification models

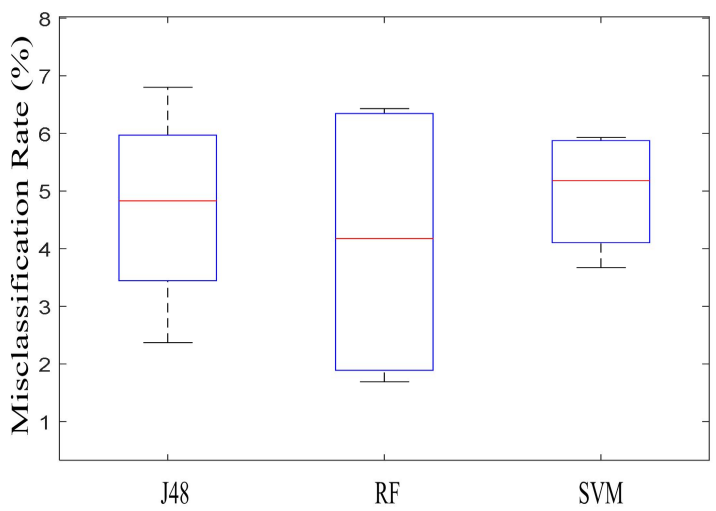

Fig. 2.7 Box plot of missclassification rate for individual classification models

\subsection{Oscillation Source Identification Results for WECC 179-System}

In addition to the validation data mentioned in previous section, additional simulations in PSS/E generated more data to evaluate the performance and scalability of the proposed ensemble learning based approach. Topology change scenarios were simulated by considering N-2 and N-3 contingencies. Also, some measurements were intentionally edited to analogue faulty or missing measurements.

Table 2.2 Classification accuracy for individual features

\begin{tabular}{|llll|}
\hline Reported Features & Classification & Accuracy & (\%) \\
& DT & RF & SVM \\
\hline \hline Generator Speed & 95.48 & 97.91 & 95.46 \\
Rotor Angle & 94.86 & 93.57 & 94.18 \\
Bus Voltage Magnitude & 97.63 & 98.31 & 96.33 \\
Bus Voltage Angle & 93.20 & 93.74 & 94.07 \\
\hline
\end{tabular}




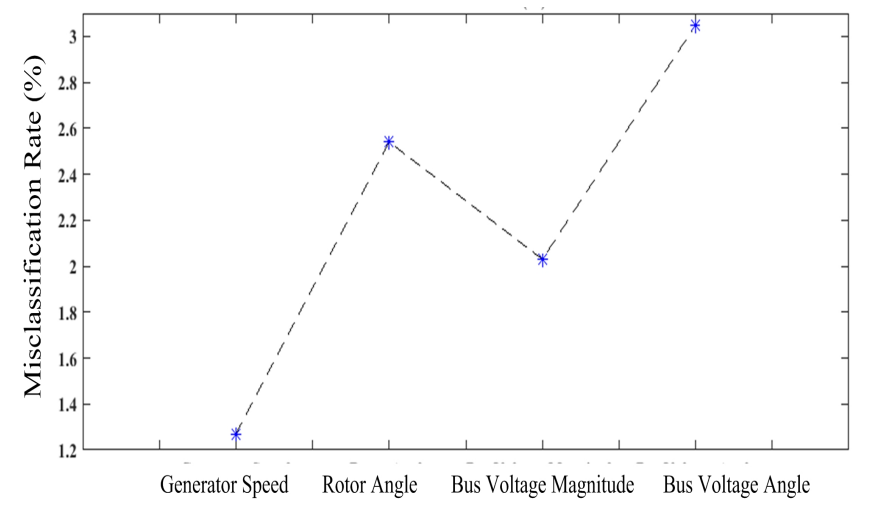

Fig. 2.8 Missclassification rate for individual reported features

\subsubsection{Additional Database Generation}

In order to check the robustness of the proposed approach and to enrich the database more scenarios were considered. An observing time window of $40 \mathrm{sec}$ was selected to cover enough observability of oscillations and loading levels $90 \%, 100 \%$ and $110 \%$ of their nominal values were considered.

1. Fault events: Three phase faults were simulated. Of the 29 generators in the system, 9 generators were selected based on their loading conditions, locations and congestions on their interconnected lines. Hence, 27 scenarios were created.

2. Clearing time: These faults were cleared at $0.03 \mathrm{sec}$ and $0.04 \mathrm{sec}$ to vary severity of faults and hence oscillatory response. Thus, 54 cases were formed.

3. N-k contingency: Not only N-1 contingencies were considered but 5 cases with $\mathrm{N}-2$ and N-3 were also simulated to analogize topology change scenarios.

4. Measurement errors: Erroneous measurements were simulated for 9 different measurement units.

The new database has additional cases with oscillations in addition to the existing 21 cases of oscillations provided in [54].

\subsubsection{Identification Results}

The performance of the proposed credibility search ensemble learning approach is evaluated for unseen real time data created in section 6.1. Oscillation stability phenomenon is relatively slow and persistent as compare to transient stability. An advantage of proposed approach is its adaptability to include new oscillation sources in its database on the identification of new source. Considering normal operating load (i.e. 100\%), the performance was evaluated for $90 \%$ and $110 \%$ loading conditions as shown in Table 
Table 2.3 CSEL implementation with different loading condition load

\begin{tabular}{|lll|}
\hline Reported Features & $\begin{array}{l}\text { Classification } \\
\text { with 90\% load }\end{array}$ & $\begin{array}{l}\text { Accuracy (\%) } \\
\text { with 110\% load }\end{array}$ \\
\hline \hline Generator Speed & 98.56 & 98.88 \\
Rotor Angle & 97.40 & 97.51 \\
Bus Voltage Magnitude & 98.01 & 97.92 \\
Bus Voltage Angle & 96.68 & 97.20 \\
\hline
\end{tabular}

2.3. As seen from the table, the location identification accuracy is highest when generator speed is selected as feature vector. Further, misclassification rate is least when generator speed vector is the feature input vector followed by the bus voltage magnitude feature vector as shown in Fig. 2.8.

\subsubsection{Impact of Topology Changes}

The proposed approach, with classification models originally trained with $\mathrm{N}-1$ contingency cases, was also tested for $\mathrm{N}-2$ and $\mathrm{N}-3$ contingencies where topology changes were involved. The performance of CSEL approach for scenarios involving a topology change can be seen in Table 2.4. The accuracy does decrease with topology changes but is still greater than $87 \%$ and it is due to the fact that training was originally done with $\mathrm{N}-1$ contingency cases only. If $\mathrm{N}-\mathrm{k}$ contingencies are considered in the original training data, where $\mathrm{k}$ depends on the robustness of the oscillation source location approach, then accuracy is speculated to improve. Hence, the results show that the proposed approach is not extremely sensitive to the topology of the training cases. The performance of the proposed approach with Gaussian measurement noise is also presented in Table 2.4 , which shows that the accuracy is not considerably affected by the measurement noise.

\subsubsection{Impact of Measurement Errors}

In training stage data with no feature vector error is used as mentioned in section 4.1, however in online stage PMU measurement error are modeled as Gaussian noise. If $n_{X}$ denotes the measurement noise then the errors are given as [56]:

Table 2.4 CSEL Performance

\begin{tabular}{|lll|}
\hline Reported Features & $\begin{array}{l}\text { Classification } \\
\text { with topology changes }\end{array}$ & $\begin{array}{l}\text { Accuracy (\%) } \\
\text { with measurement errors }\end{array}$ \\
\hline \hline Generator Speed & 88.56 & 98.84 \\
Rotor Angle & 87.83 & 96.84 \\
Bus Voltage Magnitude & 88.69 & 88.39 \\
Bus Voltage Angle & 87.36 & 97.20 \\
\hline
\end{tabular}




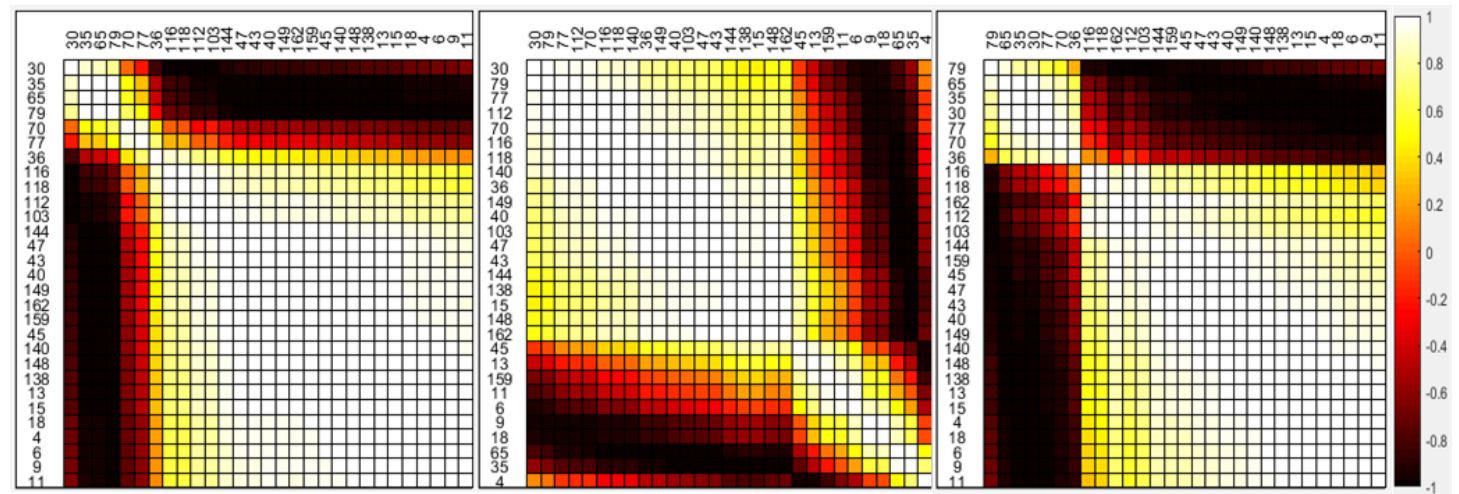

Fig. 2.9 Correlation maps for rotor angles (left) G11-source of oscillations, (center) G4-source of oscillations, (right) G11-source of oscillations for topology changes

$$
f\left(n_{X}\right)= \begin{cases}\frac{9}{\pi\left(1-e^{-9}\right) 10^{-4} X^{2}} e^{-\frac{9\left|n_{X}\right|^{2}}{10^{-4} X^{2}}} & \text { if }\left|n_{X}\right|<10^{-2} \\ 0 & \text { if }\left|n_{X}\right| \geq 10^{-2}\end{cases}
$$

The accuracy of source location identification is not affected greatly by the measurement noise as seen in Table 2.4.

\subsubsection{Comparison with Average Weight based Approach}

Classification accuracy of credibility search learning method is compared with average weights based method in Table 2.5. As seen in Table 2.5, the accuracy to locate the source of oscillations with proposed model free credibility search method is higher indicating its superiority over conventional approaches. This can be reliable tool for system operator which is important for fast and accurate remedial action.

\subsection{Discussion}

Fig. 2.9 shows three examples of oscillation source localization, where Pearson correlation among rotor angles of all 29 generators has been computed. Considering the obtained cumulative correlation of a single generator's rotor angle with all other generators, a correlation map is obtained. The generator with the least cumulative correlation

Table 2.5 Performance Comparison

\begin{tabular}{|lll|}
\hline Reported Features & $\begin{array}{l}\text { Classification } \\
\text { proposed credibility } \\
\text { search approach }\end{array}$ & $\begin{array}{l}\text { Accuracy (\%) } \\
\text { average weights based } \\
\text { approach }\end{array}$ \\
\hline \hline Generator Speed & 97.91 & 96.28 \\
Rotor Angle & 94.86 & 94.20 \\
Bus Voltage Magnitude & 98.31 & 97.42 \\
Bus Voltage Angle & 94.07 & 93.67 \\
\hline
\end{tabular}


with respect to all other generators is selected as the source of oscillation. In Fig. 2.9, G11 has the minimum correlation and hence is at the bottom of the correlation map. Similarly, rotor angle of G4 has the minimum correlation with other generators for another scenario of oscillations. G11 has the least correlation as shown in the right correlation map of Fig. 2.9 for a topology change scenario. The correlation theory and feature vector selection will be discussed in our future work.

\subsection{Evolution of Oscillations in Islanded Microgrid}

Stability in case of synchronous machines, is defined by the equilibrium of mechanical and electrical torque and depending on acceleration or deceleration of the rotor and the response of other machines, there can be loss of synchronism.

$$
J \frac{d^{2} \delta}{d t^{2}}=\tau_{m}-\tau_{e}
$$

where $\mathbf{J}$ represents inertia of the machine, $\delta$ represents the angular displacement of the rotor w.r.t. a stationary axis, $\tau_{m}$ and $\tau_{e}$ represent mechanical torque supplied by the machine the electrical torque respectively. For a small interconnected system all generators have to work in coherency for better system dynamics. Non-linear equation describing the behaviour of rotor dynamics for multiple generators working coherently can be given by:

$$
J_{e q} \frac{d^{2} \delta}{d t^{2}}=\tau_{m e q}-\tau_{e e q}
$$

where $J_{e q}$ is the equivalent inertia of the machines, $\tau_{m e q}$ represents the equivalent mechanical torque supplied by the machines, and $\tau_{e e q}$ is the net equivalent electrical torque.

On the other hand, unlike rotor dynamics of synchronous machines which is defined by swing equation (2), most of the sources in microgrid have no rotating mass and thus have no inertia or low inertia present in the system. Electric Reliability Council of Texas (ERCOT) carried out stability studies of the system with high penetration of distributed

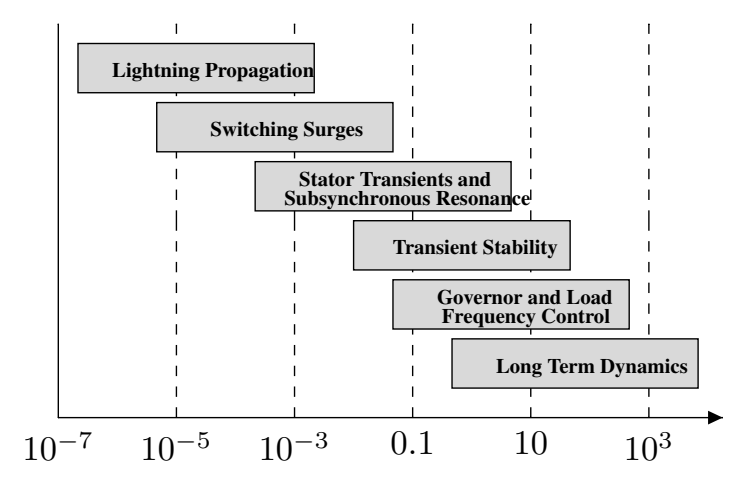

Fig. 2.10 Time ranges of dynamic phenomenons in transmission system 
generators (DGs). They observed that higher penetration of wind generation led system to its frequency nadir more quickly than system with lesser wind generation [57]. The main reason for this is the higher penetration of inverters, which leads to lower inertia of the system, and thus increase in values of $\frac{d \delta}{d t}$ as given in (2.16).

Another major difference between the sources in transmission system and microgird is their time of response following any disturbance in system. These differences calls for different stability studies to be carried out for microgrid. For instance, as shown in Fig. 2.10, line transients such as light propagation and switching surges have faster dynamics than governor of synchronous generator (SG) and hence such transients are neglected in the study of load frequency control of SG [3]. However, inverter controls and inverter dynamics are as fast as network transients, therefore network transients can not be neglected for stability studies of a microgrid system.

Inverters in an islanded microgird can be controlled in grid-forming mode or gridfeeding mode [58]. In grid-forming mode, inverters regulate the voltage and frequency of the microgrid and in grid-feeding mode inverters inject the preset real and reactive power to the microgrid. Moreover, two control techniques are widely used for inverters named as droop control and synchronverter conrol.

In droop control, inverter mimics the governor characteristics of synchronous generators. For a system operating at nominal angular frequency $\omega_{n}$, any increase in load $\Delta P_{L}$ will slow down synchronous generators $\mathrm{SG}_{1}$ and $\mathrm{SG}_{2}$, which will result in increased power output from the generators, such that $\Delta P_{1}+\Delta P_{2}=\Delta P_{L}$, and the system will reach a decreased steady-state angular frequency $\omega^{\prime}$ as shown in Fig. 2.11. Similarly, in an inverter dominated microgrid, any increase in load can be shared among inverters by decreasing the frequency based on droops, $k_{P}$ and $k_{Q}$, of individual inverters as given in (2.17). These droop controlled inverters are also named as voltage source inverters (VSI) .

$$
\begin{aligned}
\omega^{\prime} & =\omega_{n}-k_{P}\left(P^{\prime}-P\right) \\
V^{*} & =V_{n}-k_{Q}\left(Q^{\prime}-Q\right)
\end{aligned}
$$

where, $P^{\prime}$ and $Q^{\prime}$ are active and reactive power generated by inverter when it is operating at nominal (reference) voltage $V_{n}$ and frequency $\omega_{n} . k_{p}$ and $k_{Q}$ are frequency and
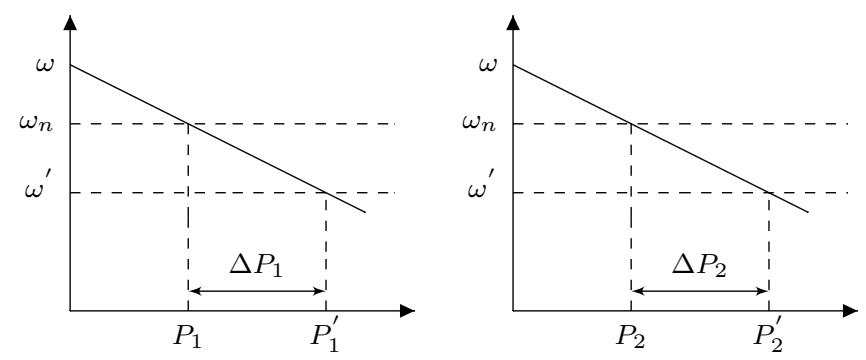

Fig. 2.11 Droop characteristics of SGs 


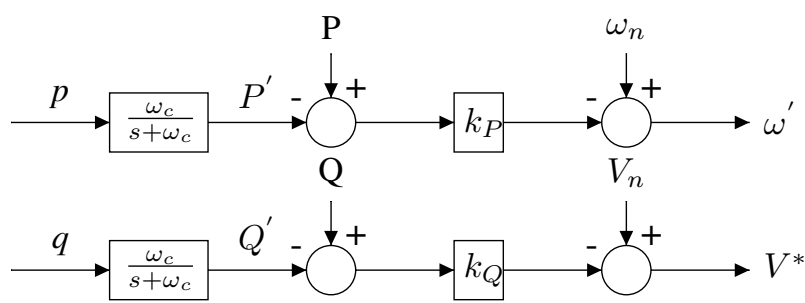

Fig. 2.12 Power controller for droop strategy

voltage droop gains respectively. This active and reactive power, $P^{\prime}$ and $Q^{\prime}$ generated by the inverter is given by:

$$
\begin{aligned}
P^{\prime} & =\frac{\omega_{c}}{s+\omega_{c}} p \\
Q^{\prime} & =\frac{\omega_{c}}{s+\omega_{c}} q
\end{aligned}
$$

where, $\omega_{c}$ is the cut-off frequency of low pass filter (LPF) which filter out the ripples present in the power calculation. Synchronverter control also uses frequency-droop and voltage-droop to control system frequency and voltage. However, in addition it uses a virtual inertia to improve the transient characteristic of the system. These inverters are named as virtual synchronous generator (VSG). The presence of such inverter controls particularly in islanded microgrid may cause stability issues owing to 1) their non-optimal droop gains $\left.\left(k_{P}, k_{Q}\right) ; 2\right)$ intermittent power/load changes due to small geographical proximity [38, 2]. [59] discussed some of these stability challenges in microgrids.

To convey an intuitive sense, a three node system having three inverters is considered as shown in Fig. 2.13. Detailed modeling of inverters' controller is done in d-q axis. The system has 47 states including inverters terminal voltage magnitude, angle, active and reactive power output etc. Small signal analysis is performed and the resulting eigenvalues of the system are plotted in Fig. 2.14. These eigenvalues can be categorized into three groups depending on the distance from the y-axis (stability axis). Eigenvalues which are closer to the y-axis are more critical since they can initiate low frequency oscillations in the system. These oscillations are sensitive to change of load (which is the most common type of disturbance in islanded microgrid) and to droop

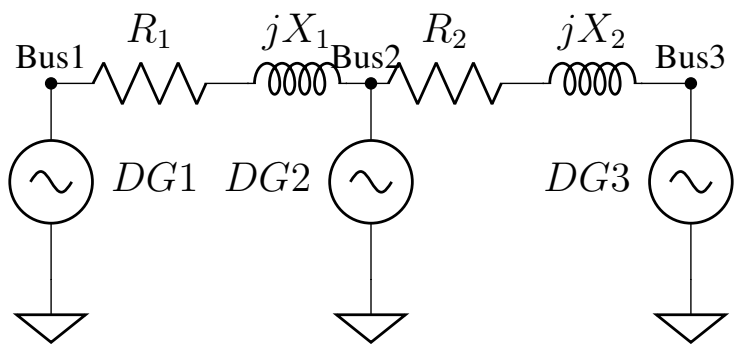

Fig. 2.13 Three bus islanded microgrid [3] 


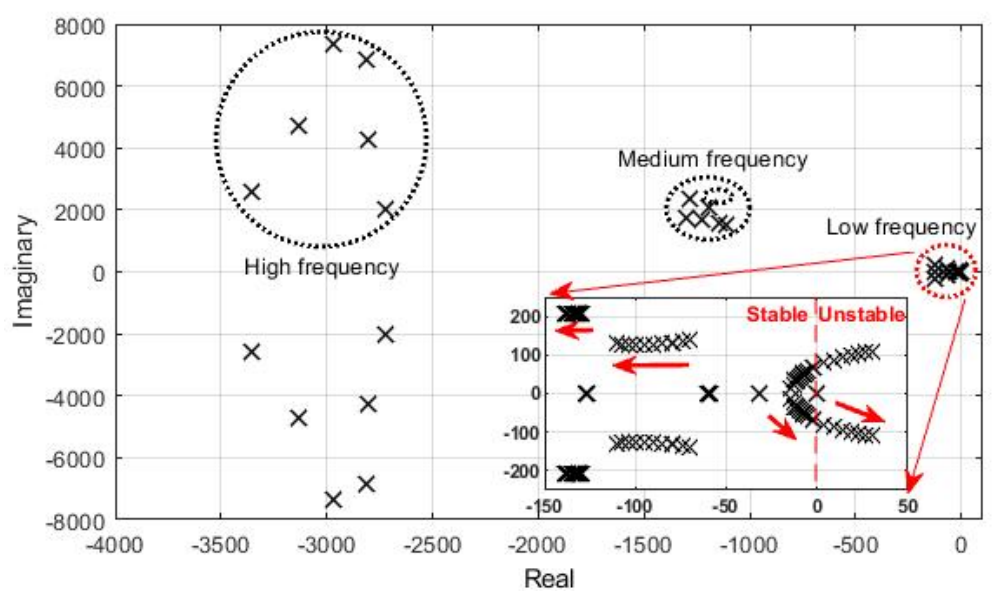

Fig. 2.14 Eigenvalue locus with varying droop gain $\mathrm{k}_{P}$

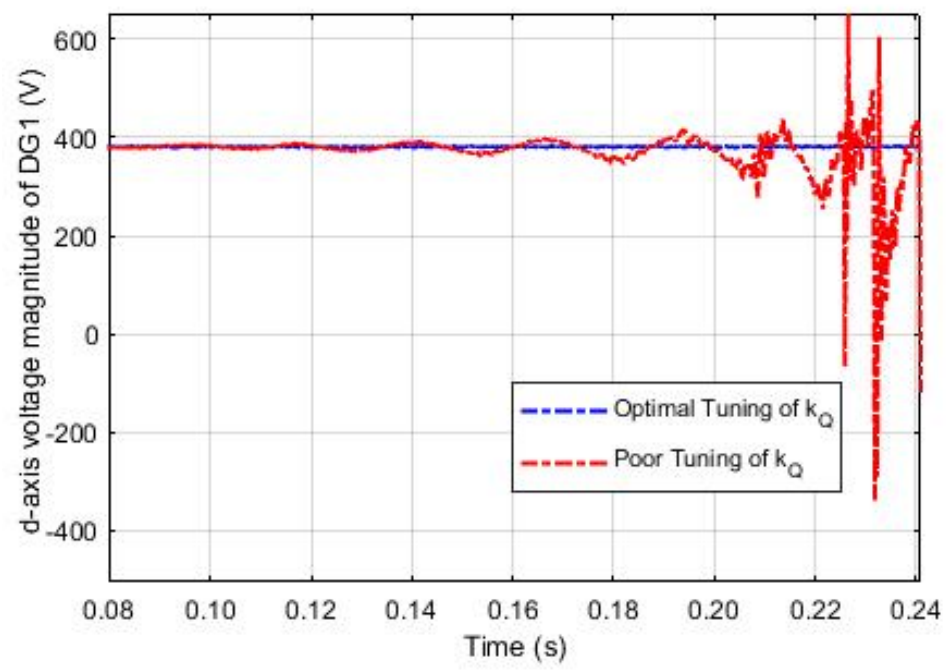

Fig. 2.15 Voltage magnitude for different values of $\mathrm{k}_{Q}$

gains $\left(\mathrm{k}_{P}, \mathrm{k}_{Q}\right)$ of the inverter. Poor choice of these droop gains may lead to undamped low frequency oscillations [3] which may also lead to system instability. Sensitivity analysis has been carried out to study the impact of droop gains on system stability. Fig. 2.14 shows the sensitivity of low frequency eigenvalues to droop gain $\mathrm{k}_{P}$. It has been observed that as $\mathrm{k}_{P}$ in (2.17) is increased, eigenvalues of system move towards imaginary axis. Sensitivity analysis shows that roots of the system moves to right hand side of imaginary axis for values of $\mathrm{k}_{p}>0.000233$. Fig. 2.15 shows the oscillations in $\mathrm{d}$ axis voltage magnitude of inverter at DG1 for poor tuning of reactive power droop gain $\mathrm{k}_{Q}$. For instance, when $\mathrm{k}_{Q}$ is at 0.0019 the system is stable, however when droop gain is altered to an improper (non-optimal) value of 0.009 , oscillations are observed and eventually system becomes unstable. Hence, it is critical to identify the source (inverter with poor droop gains), which helps mitigating these oscillations.

VSG control scheme also inherit all the advantages of droop controlled inverter; 
in addition it also provides inertia to the system[60, 61, 62]. This results in better frequency stability of the system as compared to a system consisting only droop controlled inverter[61]. However, this has not been considered in the scope of this chapter.

\subsection{Source identification Results in Islanded Microgrid}

A promising method, which has shown its practical performance in over 50 actual events from WECC and ISONE, is the Transient Energy Flow (TEF) method [12]. It tracks the net transient energy flow in all lines where measurement data is available, thus is inherently model independent. While carrying the advantage of being model free, it has few shortcomings, the most important one is that it can not distinguish between a true oscillation source bus and a bus with net "negative damping" contribution, since both are seen as sources of Transient Energy. In addition, the network is assumed to be lossless, which is very crucial for this method [41]. A comprehensive discussion on the open questions about this method can be found in [46]. Some other methods have also been presented [47], [48] but they involve solutions of high order nonlinear differential algebraic equations (DAEs). This makes them relatively computationally inefficient especially for online monitoring and decision-making. Moreover, these methods were developed and tested in transmission system, where PMU measurements at almost each bus are readily available. In addition, the presence of inertia in the form of rotating synchronous machines is also extremely helpful against these oscillations. On the other hand, with increased penetration of DERs, there is a pressing need to analyze these oscillations in islanded microgrid which is not connected or supported through large interconnected transmission system. The proximity of these DERs in a small geographical area may also generate the possibility of having more than one sources of oscillation.

It is clear that there is a need in the power systems community for the development of a more systematic approach which do not heavily rely on strong model assumptions, considers the possibility of more than one sources and more importantly analyze and identify the cause of these oscillation in an islanded microgrid. We have developed a systematic framework providing novel and efficient solution against such potentially treacherous oscillations. The specific contributions are as follows.

1. Development of an explicit algorithm that can use streaming real-time data from the measuring units to detect the source of oscillations, which may further cause instability if not properly detected and mitigated.

2. Consideration of the scenarios with more than one sources causing these oscillations.

3. Applicability of the proposed approach on oscillation cases caused from highly 


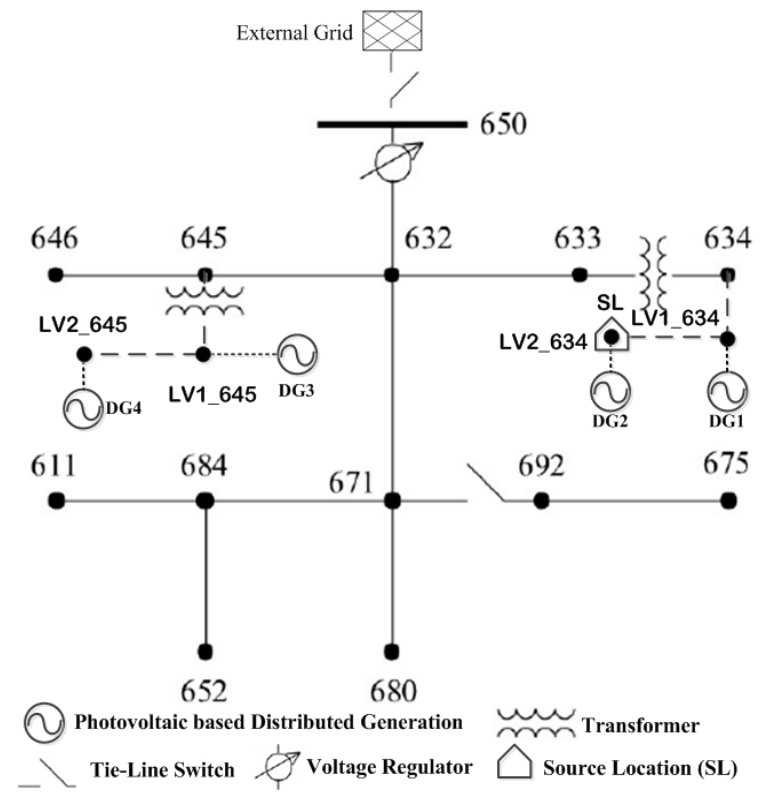

Fig. 2.16 Modified IEEE 13-node distribution network

fluctuating loads and uniquely analyzing its performance in the presence of ambient noise.

The proposed source location diagnostic framework is applied to three different scenarios utilizing modified IEEE 13-node and 34-node 3-phase unbalanced distribution networks operating in islanded mode. The performance of the proposed source identification technique is evaluated and compared with five supervised learning techniques including DT, RF, SVM, KNN and EL in terms of true positive rate (TPR) and false positive rate (FPR).

\subsubsection{Single oscillation source at the PV generator}

Figure 2.16 shows the 13-node distribution network [63] interfaced with four photovoltaic based distributed generators (DGs) and a diesel generator. Line switch closer to node 650 is opened at $\mathrm{t}=1 \mathrm{sec}$ to isolate the network from external grid and initiate islanded operation. A small periodical disturbance is applied at the node LV2 ${ }_{634}$ connected with DG2 at $\mathrm{t}=8 \mathrm{sec}$ resulting oscillations in the terminal voltage magnitudes of distributed generators $\Delta V_{D G_{i}}$ as shown in Fig. 2.17. The frequency $\left(f_{o}\right)$ of these voltage magnitude oscillations is estimated to be $0.71 \mathrm{~Hz}$ as shown in Fig. 2.18. There is an important observation regarding the variations of voltage magnitude at distributed generator terminals $\left\{\mathrm{LV}_{634}, \mathrm{LV} 2_{634}, \mathrm{LV} 1_{645}, \mathrm{LV} 2_{645}\right\}$. These variations in the time domain voltage curves do not explicitly indicate the location of the disturbance. A reliable approach is required to locate the source and hence take the appropriate countermeasures.

The synchronized phasor measurements are collected from $\mu$-PMUs installed at DG 


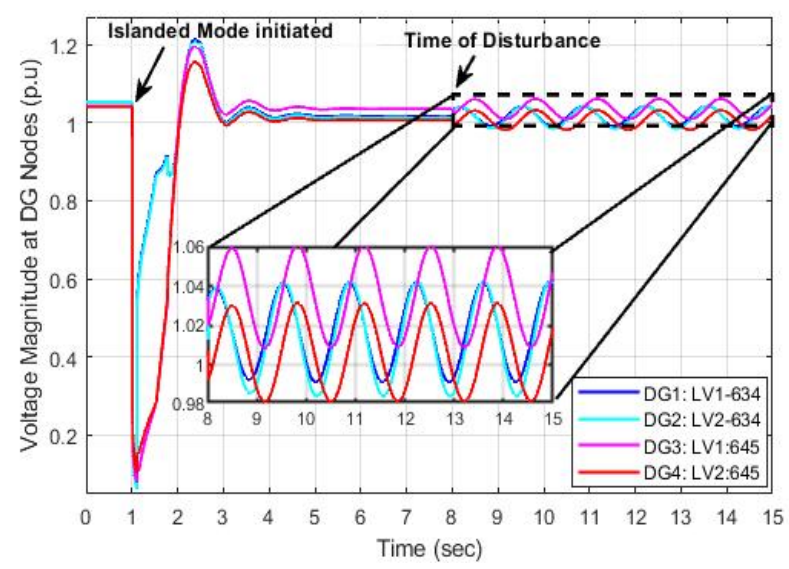

Fig. 2.17 Variations in voltage magnitude of photovoltaic based DGs

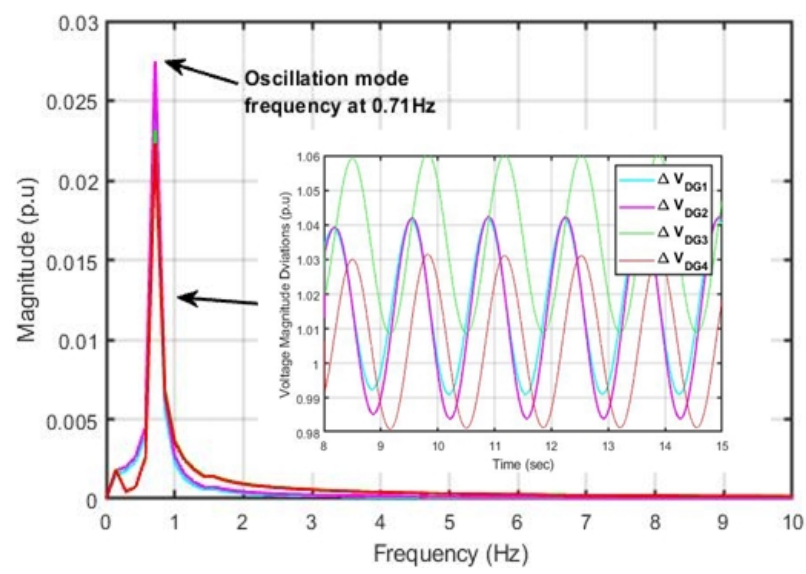

Fig. 2.18 Oscillation frequency of voltage magnitude

sites $\left\{\mathrm{LV} 1_{634}, \mathrm{LV} 2_{634}, \mathrm{LV}_{645}, \mathrm{LV} 2_{645}\right\}$. The collected dataset contain three phase voltage magnitude and angle features with the sampling frequency of $100 \mathrm{~Hz}$. In total 28 such cases are simulated including load levels $\{70 \%, 80 \%, 90 \%, 100 \%, 110 \%, 120 \%$, $130 \%\}$ for disturbance locations at distributed generators $\{\mathrm{DG} 1, \mathrm{DG} 2, \mathrm{DG} 3, \mathrm{DG} 4\}$ using DigSILENT PowerFactory. The data set is partitioned into two subsets for validation: learning and testing sets. The number of learning subsets is (k-1) and there is 1 testing subset. Learning and testing are repeated $k$ times using different (k-1) subsets each time and hence named as k-fold cross-validation. The overall accuracy (ACC) of the validation stage is computed from the average over ten folds.

To search the most credible classifier model for each reported feature, 10-fold crossvalidation is performed and the performance of the top three classifier models is reported in Fig. 2.19. It depicts the distribution of the performance measure in terms of ACC obtained by each classifier model through 10-fold cross-validation. The solid red line represents the mean ACC values. It shows RF and KNN outperform others for voltage magnitude and angle respectively. In order to analyze the sensitivity of the proposed technique two performance measures, called true positive rate (TPR) and false positive rate (FPR), are also used. Table 2.6 shows TPR and FPR results for the proposed source 


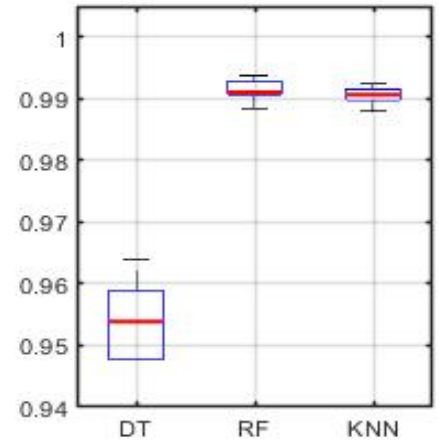

(a)

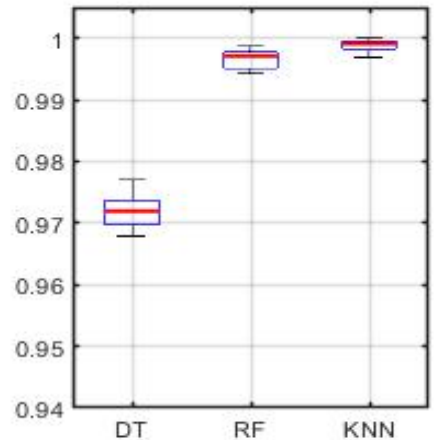

(b)

Fig. 2.19 Distribution of the attained ACC of top three classifier models during credibility search phase for voltage magnitude (a) and angle (b)

Table 2.6 TPR and FPR Results using proposed Approach

\begin{tabular}{l|ll}
\hline Measurements & TPR[\%] & FPR[\%] \\
\hline Voltage Magnitude & 98.26 & 0.58 \\
\hline Voltage Angle & 99.04 & 0.32 \\
\hline
\end{tabular}

identification framework.

We also validate the effectiveness of the proposed data driven approach on bigger unbalanced 34-node distribution network integrated with photovoltaic distributed generators $\{$ DG1, DG2, DG3, DG4, DG5, DG6, DG7 $\}$ at nodes $\{800,812,822,830,846$, $862,864\}$ and operating in islanded mode. The network diagram of the modified unbalanced 34-node distribution system is shown in Fig. 2.20. Switch located at node 800 is opened at $\mathrm{t}=3 \mathrm{sec}$ to isolate the network from the external grid. A small periodical disturbance is applied at the node 830 connected with DG4 at $\mathrm{t}=8 \mathrm{sec}$ resulting oscillations in voltage magnitude at the coupling nodes of distributed generators $\Delta V_{D G_{i}}$

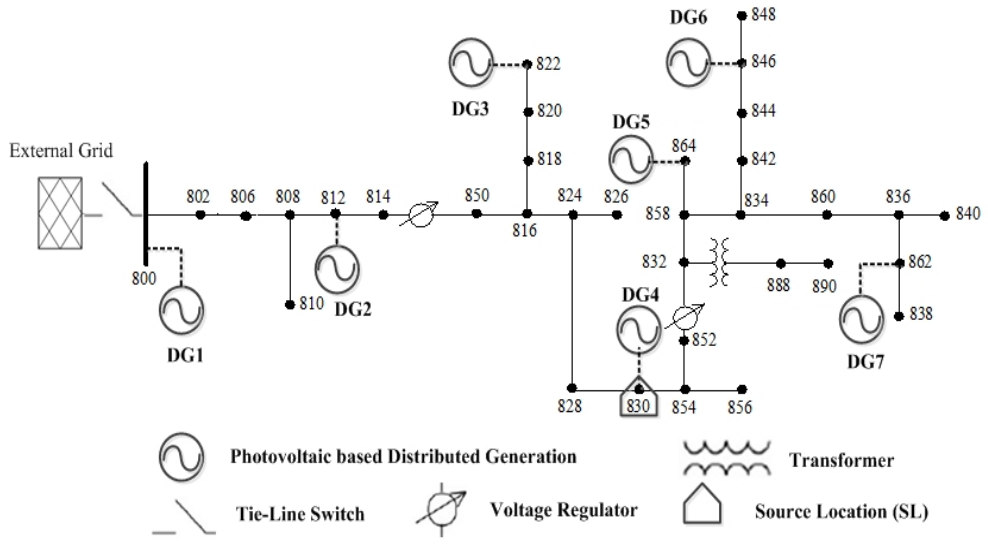

Fig. 2.20 Modified IEEE 34-node distribution network 


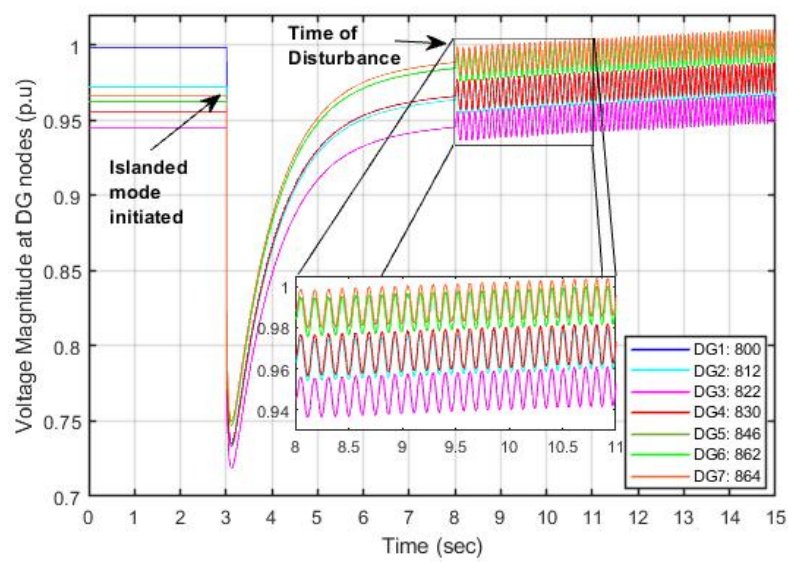

Fig. 2.21 Variations in voltage magnitude of photovoltaic based DGs

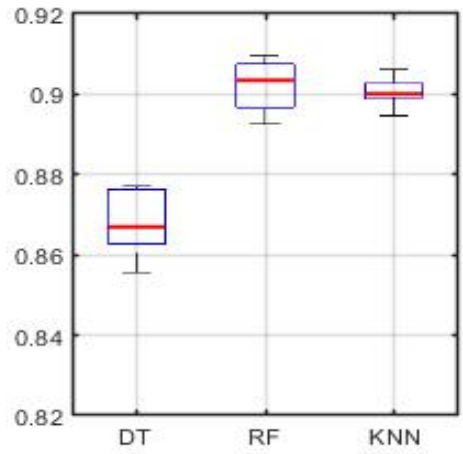

(a)

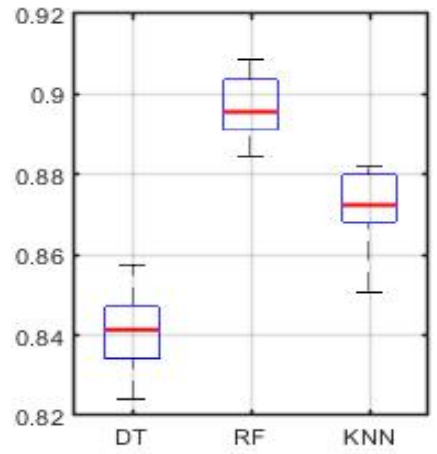

(b)

Fig. 2.22 Distribution of the attained ACC of top three classifier models during credibility search phase for voltage magnitude (a) and angle (b)

as shown in Fig. 2.21. Time synchronized voltage phasor measurements are collected from $\mu$-PMUs installed at DG sites $\{800,812,822,830,846,862,864\}$. 25 scenarios are simulated including load levels $\{80 \%, 90 \%, 100 \%, 110 \%, 120 \%\}$ for disturbance locations at distributed generators $\{\mathrm{DG} 1, \mathrm{DG} 2, \mathrm{DG} 3, \mathrm{DG} 5, \mathrm{DG} 6\}$.

The performance of the most credible classifier in terms of ACC, for each reported feature, is obtained through 10-fold cross-validation and shown with box-plots in Fig. 2.22. The distribution of ACC shows RF outperforms DT and KNN for both voltage magnitude and angle. Table 2.7 shows the sensitivity measures results in terms of TPR and FPR for unbalanced 34-node network operating in islanded mode and integrated with photovoltaic distributed generators.

Moreover, statistical significance tests are conducted on the attained results to further analyze and investigate if the proposed novel approach could significantly improve TPR and FPR measures. A post-hoc test, called Nemenyi [64] is utilized to determine significant differences and are presented in the critical difference (CD) diagram. It illustrates the averaged rank attained by each technique and the significant differences 
Table 2.7 TPR and FPR Results using proposed Approach

\begin{tabular}{l|ll}
\hline Measurements & TPR[\%] & FPR[\%] \\
\hline Voltage Magnitude & 90.30 & 2.43 \\
\hline Voltage Angle & 89.50 & 2.62 \\
\hline
\end{tabular}

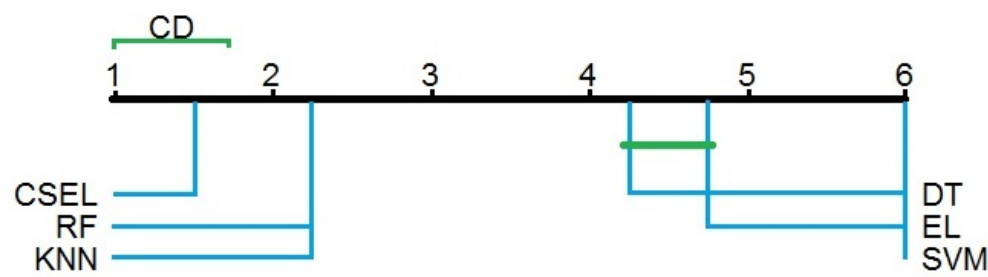

(a)

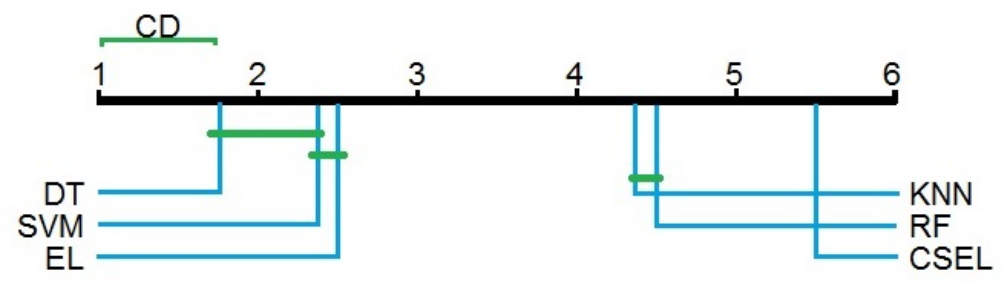

(b)

Fig. 2.23 CD diagrams for the nemenyi tests; Comparing each source identification technique in terms of TPR (a) and FPR (b) for the single oscillation source scenarios

among them. Techniques which are not significantly different are connected.

Figure 2.23 illustrates CD diagrams in terms of TPR and FPR obtained through each source identification technique. The panel (a) shows that the proposed CSEL technique attains the highest rank in terms of TPR and significantly outperforms others. DT and EL are ranked 4th and 5th respectively with a slight difference between them and thus are connected by a green solid line together. The panel (b) indicates that CSEL attains the lowest rank in terms of FPR which implies that it has the minimum false positive rate prediction. in other words, it performs better than other techniques in terms of FPR. $\mathrm{RF}$ and KNN have similar performance in terms of FPR and thus are connected with a solid green line in the CD diagram. Similarly SVM and EL share the same significance level in terms of FPR as shown in panel (b) of Fig. 2.23. The obtained results show that CSEL outperforms other techniques and provides the best performance for single oscillation scenarios in 13 and 34 node distribution networks.

\subsubsection{Multiple oscillation sources at PV generators}

The presence of multiple oscillation sources can not be ruled out due to co-existence of multiple distributed generators in a feasible geographical proximity. Thus, the challenge of identifying existing multiple disturbance sources for oscillations needs to be considered and validated by a reliable and resilient source detection approach. To validate the applicability of the proposed source identification technique for multiple os- 


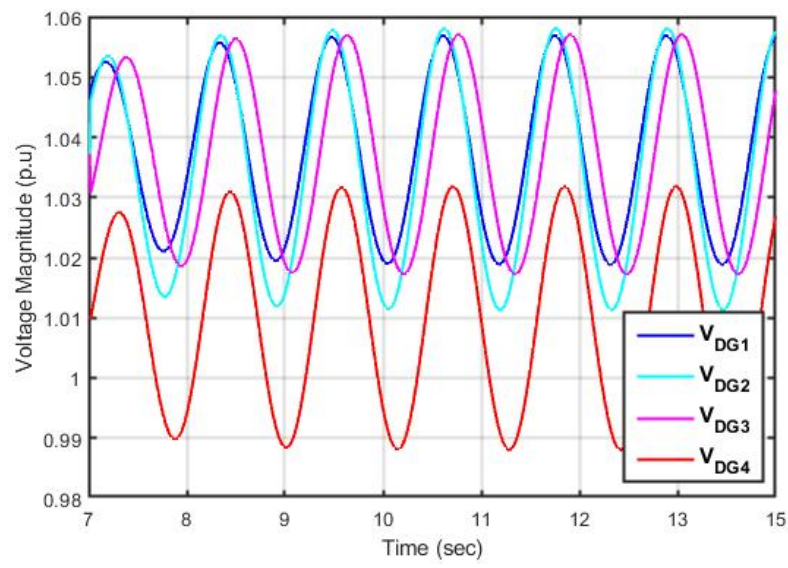

Fig. 2.24 Variations in voltage magnitude of photovoltaic based DGs

cillation sources, we simulated these cases by injecting more than one periodical disturbances. For instance, in the modified 13-node distribution network operating in islanded mode, periodical disturbances are injected, at $\mathrm{LV} 2_{634}$ and $\mathrm{LV} 2_{645}$ connected with DG 2 and DG4 respectively, at $\mathrm{t}=7 \mathrm{sec}$. Oscillations in the voltage magnitude $\Delta V_{D G_{i}}$ and angle $\Delta \delta_{D G_{i}}$ at the coupling nodes of distributed generators $\left\{\mathrm{LV} 1_{634}, \mathrm{LV} 2_{634}\right.$, $\left.\mathrm{LV}_{645}, \mathrm{LV} 2_{645}\right\}$ are observed as shown in Fig. 2.24. Synchronized voltage phasor measurements are collected from $\mu$-PMUs installed at DG sites $\{800,812,822,830$, $846,862,864\}$. Frequency of oscillations associated with this simulated scenario and loading condition is $0.85 \mathrm{~Hz}$. In addition, a comparative analysis of oscillation frequency and loading conditions was also conducted. For that four loading conditions $\{90 \%, 100 \%, 110 \%, 120 \%\}$ were considered and frequency of the resulting oscillations
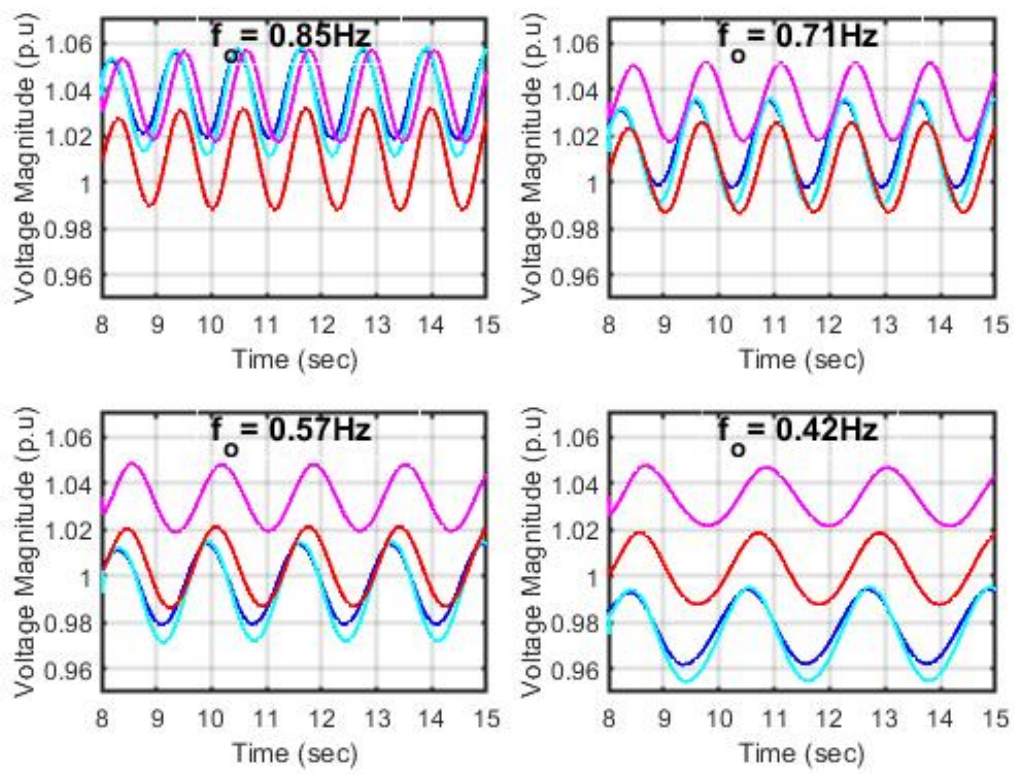

Fig. 2.25 voltage magnitude variations for different frequency modes 


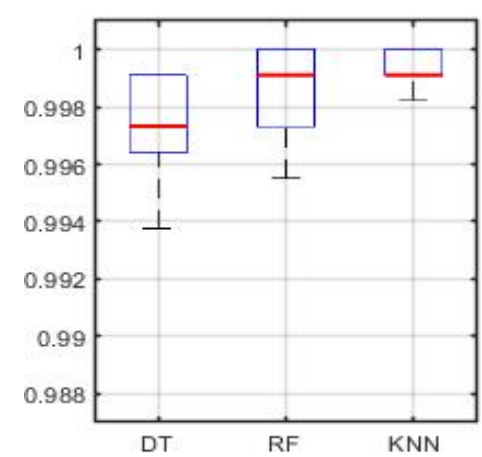

(a)

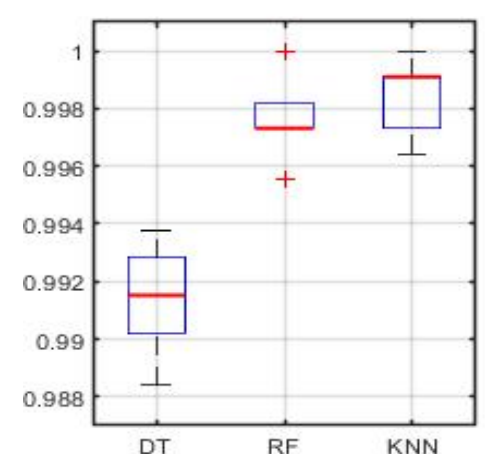

(b)

Fig. 2.26 Distribution of the attained ACC of top three classifier models during credibility search phase for voltage magnitude(a) and angle (b)

Table 2.8 TPR and FPR Results using proposed Approach

\begin{tabular}{l|ll}
\hline Measurements & TPR[\%] & FPR[\%] \\
\hline Voltage Magnitude & 98.58 & 0.71 \\
\hline Voltage Angle & 99.47 & 0.27 \\
\hline
\end{tabular}

was monitored. It is to be noted that with the increase of the load levels i.e. moving from from $90 \%-120 \%$, the oscillation frequency decreases as depicted in Fig. 2.25.

21 cases, with system loading of 70\%-130\% with increment of $10 \%$, for disturbance locations at distributed generators $\{(\mathrm{DG} 1, \mathrm{DG} 3),(\mathrm{DG} 2, \mathrm{DG} 4),(\mathrm{DG} 1, \mathrm{DG} 4)\}$ are simulated. The collected dataset contain three phase voltage magnitude and angle features. The most credible classifier model, in terms of ACC, for each feature is determined through 10-fold cross-validation and reported in Fig. 2.26. ACC distribution shows KNN outperforms others for both voltage magnitude and angle. In addition, the sensitivity of the proposed technique in terms of TPR and FPR is also computed and reported in Table 2.8 .

For the modified 34-node distribution network operating in islanded mode, periodical disturbances are injected, at 812 and 846 connected with DG2 and DG6 respectively, at $\mathrm{t}=8 \mathrm{sec}$. Oscillations in voltage magnitude $\Delta V_{D G_{i}}$ and angle $\Delta \delta_{D G_{i}}$ at coupling nodes of DGs $\{800,812,822,830,846,862,864\}$ are monitored through $\mu$-PMUs. Oscillations as high as 3.5 p.u in voltage magnitude were observed as shown in Fig. 2.27. Such high magnitude fluctuations in voltage can cause system's equipment vibrations and hence reduce their lifetime. Thus the source identification and mitigation of oscillation is inevitably critical. For credibility search phase, 20 scenarios, with system loading of $80 \%-120 \%$ with increment of $10 \%$, for disturbance location sets at $\{$ (DG1, DG4), (DG2, DG5), (DG2, DG6), (DG3, DG7)\} were simulated. The most credible classifier model, in terms of ACC, for each feature identified through 10-fold crossvalidation is reported in Fig. 2.28. It shows RF is the most credible classifier model 


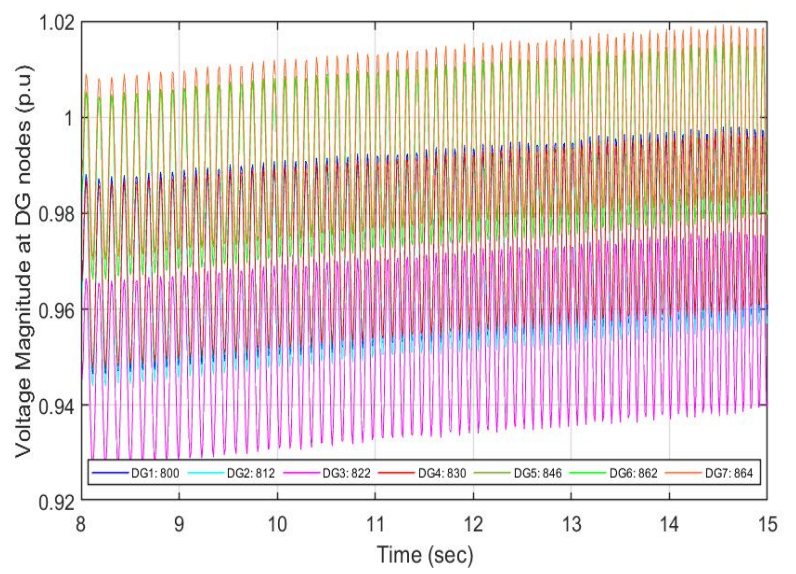

Fig. 2.27 Variations in voltage magnitude of Photovoltaic based DGs

for both voltage magnitude and angle features and can predict the disturbance source location accurately. Further, the sensitivity measures of the proposed technique are also computed and reported in Table 2.9.

Statistical significance test is conducted to investigate if the proposed novel technique could significantly improve the performance. Nemenyi test is applied to determine significant differences and the ranking results are presented in the CD diagram as shown in Fig. 2.23. The panel (a) shows that the proposed CSEL technique attains the highest rank in terms of TPR. KNN is ranked 2nd, however it's performance in terms of TPR is statistically very closer to CSEL and that is why it is connected to CSEL with

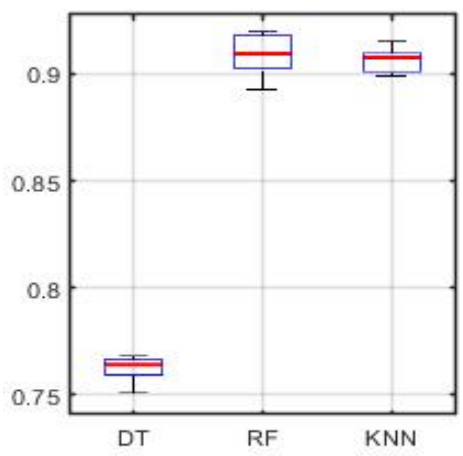

(a)

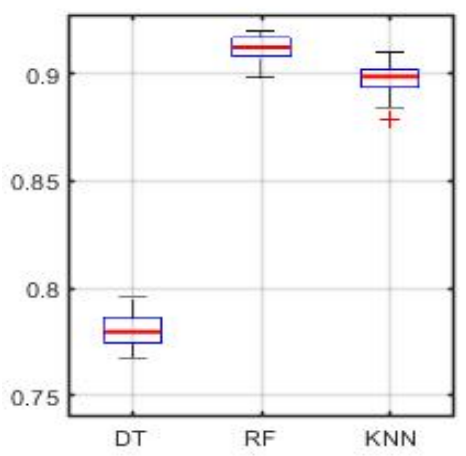

(b)

Fig. 2.28 Distribution of the attained ACC of top three classifier models during credibility search phase for voltage magnitude(a) and angle (b)

Table 2.9 TPR and FPR Results using proposed Approach

\begin{tabular}{l|ll}
\hline Measurements & TPR[\%] & FPR[\%] \\
\hline Voltage Magnitude & 91.44 & 2.86 \\
\hline Voltage Angle & 90.69 & 3.10 \\
\hline
\end{tabular}




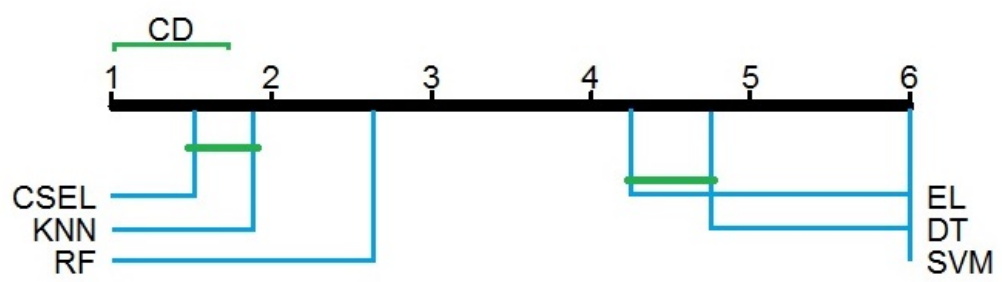

(a)

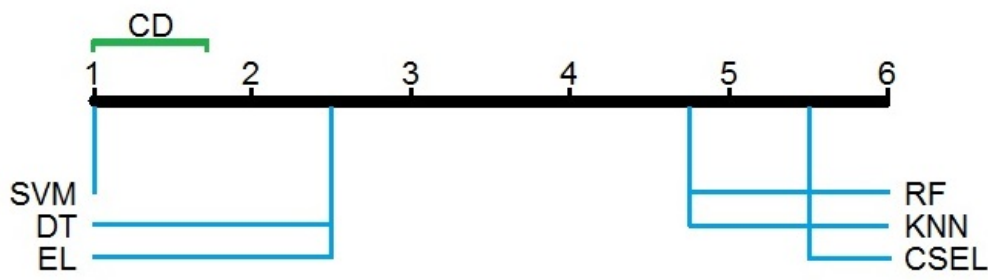

(b)

Fig. 2.29 CD diagrams for the nemenyi tests; Comparing each source identification technique in terms of TPR (a) and FPR (b) for multiple sources

a solid green line. Similarly EL and DT are connected together as they have slight difference in their TPR performance. The panel (b) indicates that CSEL attains minimum FPR and hence ranked last. It implies that it has the minimum false positive rate prediction and performs better than other techniques in terms of FPR. These results signify that CSEL performs better than other techniques multiple oscillation sources.

\subsubsection{Oscillation source at highly fluctuating cyclic load}

In real power system, the sudden variation of load may also result in low frequency oscillations in the system [65]. One challenge in the microgrid controls is its high susceptibility towards oscillations resulting from these fluctuating loads. Hence, we further validate the effectiveness of the proposed source identification approach for oscillations originated due to fluctuating loads. Periodic disturbance is injected atload $\mathrm{L}_{646}$ at $\mathrm{t}=7 \mathrm{sec}$ in 13-node distribution network operating in islanded mode. Oscillations in voltage magnitude $\Delta V_{D G_{i}}$ at $\mathrm{DG}$ coupling nodes $\left\{\mathrm{LV} 1_{634}, \mathrm{LV} 2_{634}, \mathrm{LV} 1_{645}, \mathrm{LV} 2_{645}\right\}$ are observed as shown in Fig. 2.30. It has been observed that two sets of DGs (DG1 and DG2) and (DG3 and DG4) respond to the disturbance differently w.r.t each other as can be seen from their voltage magnitude response in Fig. 2.30. On the other hand, DG1 and DG2 have similar response as well as DG3 and DG4 show a similar pattern in their voltage magnitude response. Generating units which respond to a disturbance in a similar manner are said to be coherent and are very crucial for controlled islanding $[66,67,68]$. It helps maintaing transient stability and minimizing the catastrophic affects of cascading failures [69]. In the given disturbance scenario, an effective controlled islaning scheme can roughly look like the one shown in Fig. 2.31. However, the complete dis-aggregation of the network into independent sub-networks depends on 


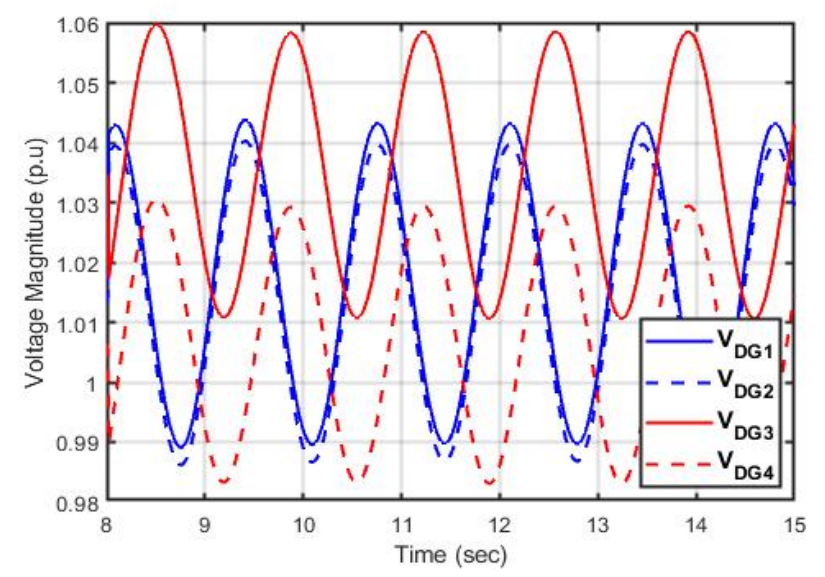

Fig. 2.30 Variations in voltage magnitude of photovoltaic based DGs

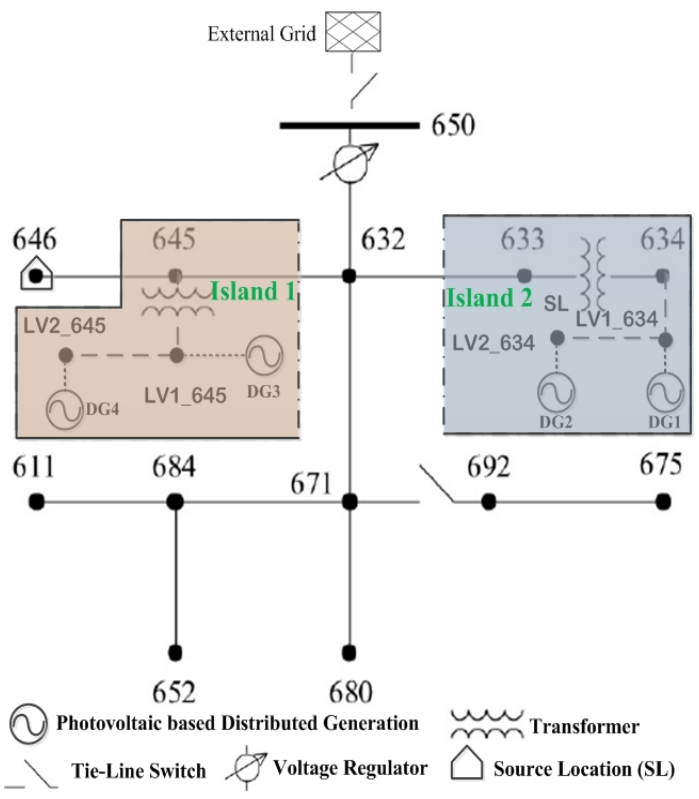

Fig. 2.31 Coherency based islanding scenario for emergency condition

many other factors in addition to coherency and its beyond the scope of this work.

Time synchronized voltage phasor measurements are collected from $\mu$-PMUs installed at these DG sites. 14 cases, with system loading of 70\%-130\% with increment of $10 \%$, for disturbance locations at node 646 and 671 are simulated. The collected dataset contains three phase voltage magnitude and angle features. To find the most credible classifier model for each of these features, 10-fold cross-validation is performed and the performance distribution for these 10 folds in terms of ACC is reported in Fig. 2.32. RF performs better for voltage magnitude and $\mathrm{KNN}$ shows highest ACC for voltage angle. Further, sensitivity of the proposed technique in computed in terms of TPR and FPR as reported in Table 2.10.

Effectiveness of the proposed source identification method for oscillations originated due to fluctuating loads is also tested for modified 34-node distribution network 


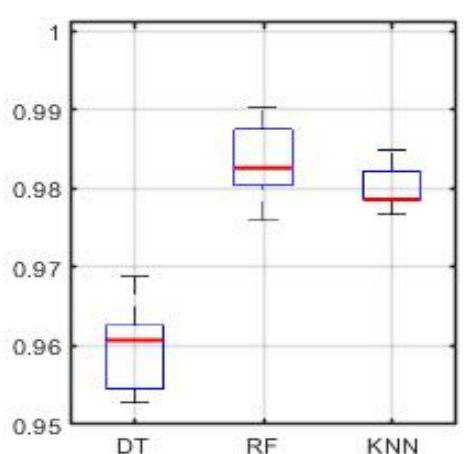

(a)

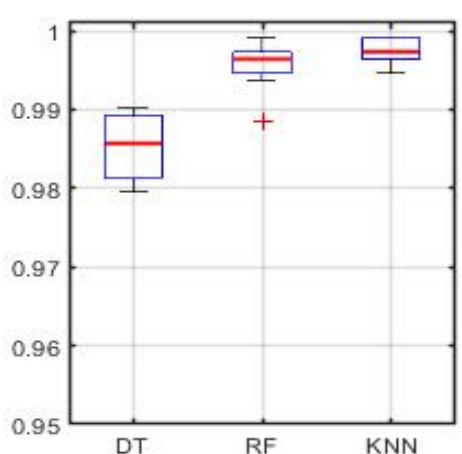

(b)

Fig. 2.32 Distribution of the attained ACC of top three classifier models during credibility search phase for voltage magni-tude(a) and angle (b)

Table 2.10 TPR and FPR Results using proposed Approach

\begin{tabular}{l|ll}
\hline Measurements & TPR[\%] & FPR[\%] \\
\hline Voltage Magnitude & 98.45 & 2.70 \\
\hline Voltage Angle & 99.65 & 0.15 \\
\hline
\end{tabular}

operating in islanded mode. Periodical disturbance is injected at node 840 at $\mathrm{t}=8 \mathrm{sec}$. Oscillations in voltage magnitude $\Delta V_{D G_{i}}$ and angle $\Delta \delta_{D G_{i}}$ at coupling nodes of DGs $\{800,812,822,830,846,862,864\}$ are monitored through $\mu$-PMUs as shown in Fig. 2.33. 15 scenarios including load levels $80 \%-120 \%$ with increments of $10 \%$, for disturbance at load nodes 810,840 and 890 were simulated. Voltage magnitude $V_{D G_{i}}$ and angle $\delta_{D G_{i}}$ measurements on DG sites $\{800,812,822,830,846,862,864\}$ were recorded. Further, 10-fold cross validation is utilized to find the most credible classifier model for each feature. Fig. 2.34 indicates that RF is the most credible classifier model, in terms of ACC, for voltage magnitude and angle and can predict the disturbance source location accurately. Additionally, sensitivity measures of the proposed novel technique are also determined and listed in Table 2.11 .

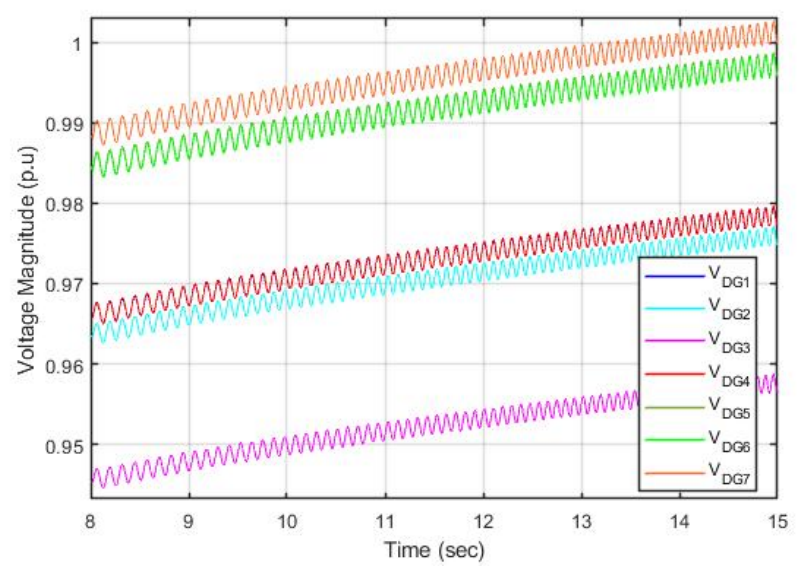

Fig. 2.33 Variations in voltage magnitude of photovoltaic based DGs 


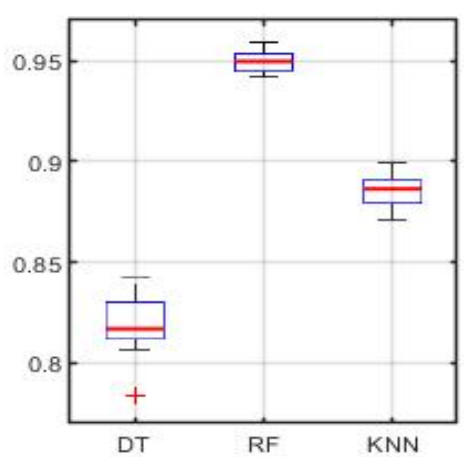

(a)

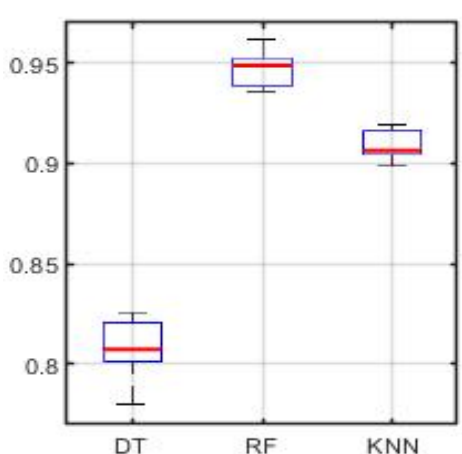

(b)

Fig. 2.34 Distribution of the attained ACC of top three classifier models during credibility search phase for voltage magni-tude(a) and angle (b)

Table 2.11 TPR and FPR Results using proposed Approach

\begin{tabular}{l|ll}
\hline Measurements & TPR[\%] & FPR[\%] \\
\hline Voltage Magnitude & 94.92 & 2.54 \\
\hline Voltage Angle & 94.75 & 2.63 \\
\hline
\end{tabular}

Statistical significance test is also conducted to investigate if the proposed novel technique could significantly improve the performance. Nemenyi test is applied to determine significant differences and the ranking results are presented in the CD diagram as shown in Fig. 2.35. Panel (a) shows that the proposed CSEL technique attains the highest rank in terms of TPR. DT and EL are ranked 4th and 5th respectively with a slight difference between them and thus are connected with a green solid line together. The panel (b) indicates that CSEL attains minimum FPR and hence ranked last, which

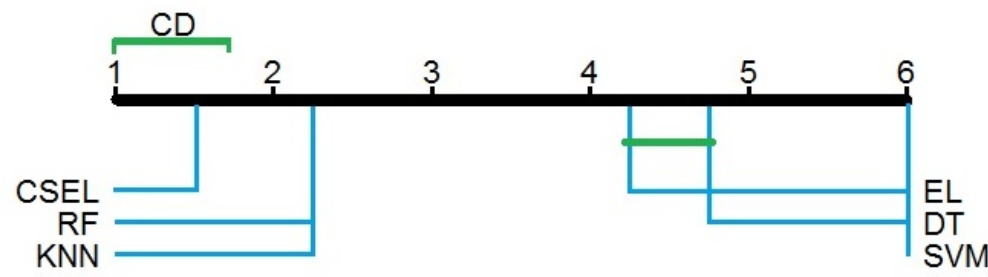

(a)

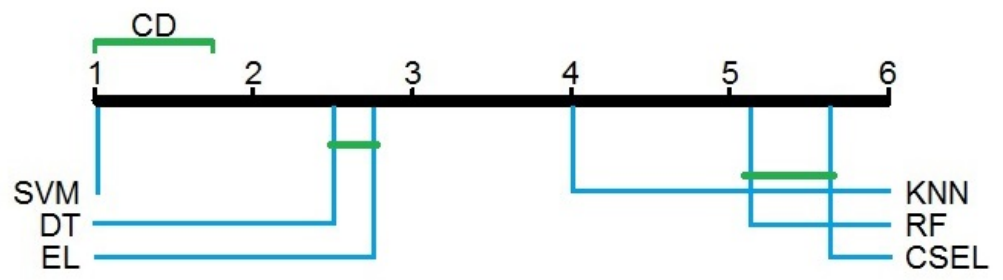

(b)

Fig. 2.35 CD diagrams for the nemenyi tests; Comparing each source identification technique in terms of TPR (a) and FPR (b) for the oscillation caused by fluctuating loads 


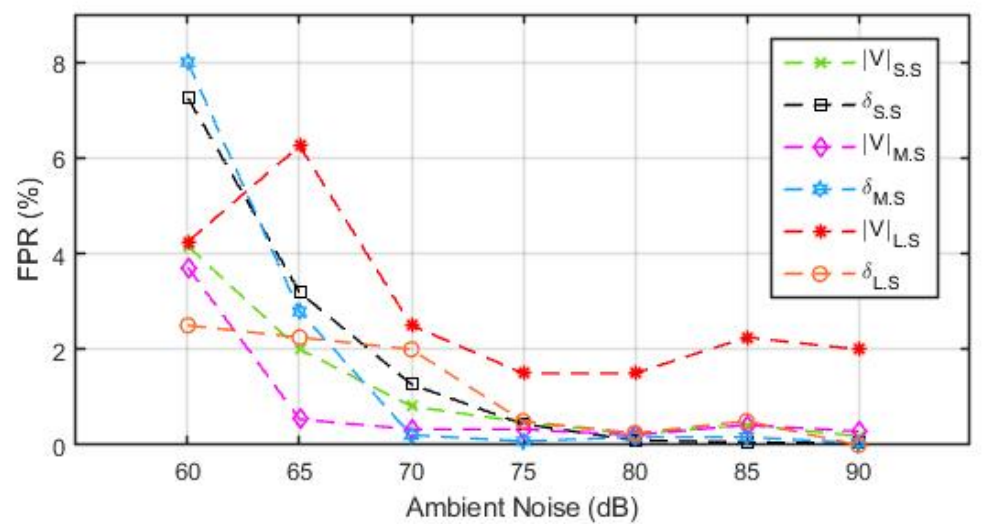

Fig. 2.36 False positive rate for different measurement noise levels in $\mathrm{dB}$

implies that it has the minimum false positive rate prediction and performs better than other techniques in terms of FPR. DT and EL are ranked 2nd and 3rd respectively but $\mathrm{CD}$ diagram connects them together due to slight difference between their performance. These results signify that CSEL performs better than other techniques for scenarios which involves oscillations caused by fluctuating loads.

\subsubsection{Performance in the presence of ambient noise}

To validate the applicability and robustness of the developed oscillation source identification approach for actual $\mu$-PMU measurements in the presence of noise, white Gaussian noise is added for simulating the measurement noise. Voltage magnitude and angle measurements are collected from $\mu$-PMUs and white Gaussian noise is added with SNR from $90 \mathrm{~dB}$ to $60 \mathrm{~dB}$ with a step of $5 \mathrm{~dB}$ (i.e. SNR $\in$ [60dB, 90dB]). Fig. 2.36 shows the results of FPR, for three cases considered, with different ambient noises. It can be seen that the FPR is not drastically deteriorated with the significant increase in the noise level. This observation can validate the robustness of the developed approach with respect to certain level of ambient noises.

\subsection{Conclusion}

In summary, this work identifies the source cause of low frequency oscillations in an islanded microgrid. We have developed an explicit approach that can use streaming real-time $\mu$-PMU data to detect the source, which may further cause instability if not properly detected and mitigated. The developed approach has also being validated for oscillation scenarios with more than one sources, causing these oscillations. We have shown its applicability on oscillation cases caused from highly fluctuating loads through the case studies provided in Section IV. We have also exhibited that the method is robust to PMU noise, and free of strong modeling assumptions, meaning that the accurate 
knowledge of system model is not a binding requirement. 


\section{CHAPTER 3}

\section{Data Driven Coherence Identification using Dynamic Time Warping for Controlled Islanding}

\subsection{Introduction}

The expansion of power grid due to regional interconnections and the increase in diversity of the transmission structure owing to ever increasing market competition have made safety and stability of the system operation crucial. Not only disturbances caused by natural calamities such as hurricanes and earthquakes but also operational mistakes may trigger cascading failures, which may result in system-wide blackouts and pose a significant threat to properties and lives [70].

Controlled islanding is a practical approach to prevent system-wide instabilities and blackouts. It splits a power system into smaller subsystems, referred to as islands. The objective is to form stable islands by selecting an optimal set of lines to disconnect while minimizing generation/load imbalance, maintaining voltage stability, ensuring generators coherency, and restraining out-of-step oscillations.

The stability of each island depends on the coherency of generators on it. Therefore, correct and adaptive identification of generators coherency is essential. Moreover, the coherency between groups of generators varies over time, due to changing network topology and operating conditions, necessitating real-time coherency determination [71]. With the increasing deployment of phasor measurement units, online measurement-based coherency identification has become feasible.

There is substantial literature on generator coherency identification. A model based continuation method was discussed in [72], which demonstrated that generator coherence changes with system network topology and operating conditions. Another modelbased eigenvalue analysis approach was presented in [73]. However, both approaches required precise knowledge of system models, parameters, and states, which are generally difficult to obtain in practice. The slow coherency-based analysis proposed in [74] was an offline model-based approach. Variations in system's conditions and topology may change the grouping of coherent generators. This behavior is associated with the generators loose coherency characteristic, and one coherent generator group may split into sub-groups, or multiple coherent groups may join a bigger coherent group 
[75]. For this reason, identifying coherent generator groups during real-time operation, based on the current system conditions, is preferred as compared to defining it offline. In addition, the slow coherency-based analysis is based on the linearized electromechanical model of a given system, which may fail under certain conditions due to high non-linearity of power systems.

In [76], coherent generator groups were identified using discrete Fourier analysis. Internal voltage phasors of generators were estimated by using voltage and current phasors measured by phasor measurement units (PMUs) at generator terminals. Jonsson et al. further improved this method by combining generator speed with Fourier analysis [77] where Inter-area dominant modes were dentified as Fourier coefficients with the most significant amplitude. However, Fourier analysis based approaches assume linearity and stationarity of the data, which does not hold for inter-area oscillations. Principal component analysis (PCA) method proposed in [78] employed bus voltage angle and generator speed for coherency identification but required prior information of system dynamic characteristics, which is difficult to obtain. A correlation coefficient based method as proposed in [79] did not require prior information as PCA method did, but a threshold to identify the correct number of coherent groups, which may vary for different operating conditions and fault locations. The threshold requires expert system knowledge which makes practical implemention of the approach challenging. Another method in [80] used bioinformatics clustering technique which required prespecification of the number of clusters. Ariff et al. presented an approach based on independent component analysis [81]. Another measurement-based approach, using artificial neural network (ANN) was introduced in [82] which needed excessive offline training. For large interconnected networks, consideration of all possible groups for offline training is daunting. In addition, real-time and large volume of PMU data interchange bring forth additional complications in case of communication failure. Succinctly an algorithm that can achieve online coherency with limited system knowledge and is robust to partial data loss is the need of the hour.

In view of this, the chapter proposes an approach for online coherency determination that also handles partial observability of the system. It provides an adaptive option to system operators for intentional islanding operation to minimize the impact of cascading outages. The enormous success of dynamic time warping [83], [84], [85] for pattern matching tasks encourages its use for generators clustering. The proposed approach has been compared and contrasted with correlation [86] and community detection [71] based approaches for the IEEE 39-bus system. It has also been compared with hierarchical clustering (HC) [87] and ICA [81] for Western interconnection power system in North America (WECC) 179-bus system. Time domain simulations are used to validate and demonstrate the effectiveness of the proposed methodology in minimizing impacts of cascading outages and system-wide blackouts. 


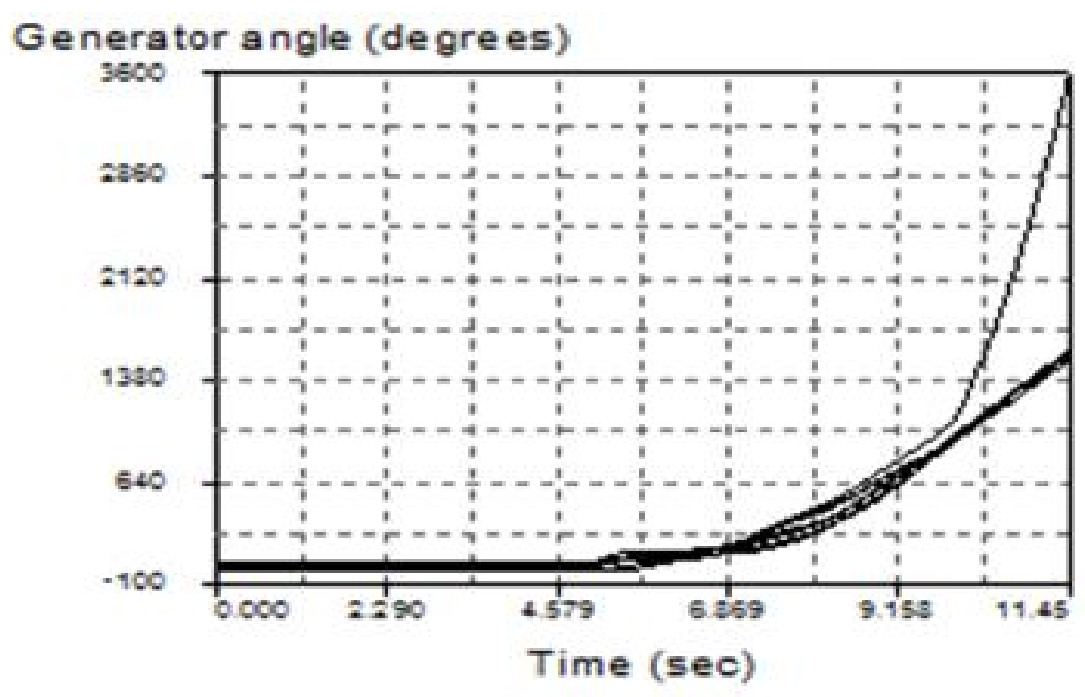

Fig. 3.1 Generators response following cascaded outages

\subsection{Adaptively controlled islanding framework}

Cascading outages can initiate electromechanical oscillations in power systems. As shown in Fig. 3.1, two cascaded outages occurred at $\mathrm{t}=5 \mathrm{~s}$ and $\mathrm{t}=7 \mathrm{~s}$, and one generator lost synchronism. The system eventually became unstable at $\mathrm{t}=11.45 \mathrm{~s}$. An efficient islanding scheme should separate generators with different behavior and ensure that coherent generators remain on the same island: to improve the transient stability; to reduce the chances of further outages.

The proposed adaptive controlled islanding scheme can be implemented using following steps.

Step 1: Estimation of generators' rotor angles based on PMU measurements of voltage and current at each generator terminal bus.

Step 2: Similarity evaluation between generators rotor angle responses using algorithm proposed in Section 3.2.1. It defines a matrix of similarity index for each pair of generators.

Step 3: Optimal number of coherent groups (k) selection by minimizing inter-coherent group distances [88]. It provides the number of unique coherent groups.

Step 4: Grouping of generators using k-means into k coherent groups, obtained from Step 3, and building a coherency constraint matrix Q using (3.6).

Step 5: Building edges' weight matrix W and Laplacian matrix L using (3.4) and (3.5) respectively.

Step 6: Formatting the grid as a graph $\mathrm{G}=(\mathrm{V}, \mathrm{E}, \mathrm{W})$ using power flow results. 
Step 7: Solving constrained optimization problem in (3.7) by finding eigenvalues in (3.8).

Step 8: Ignore eigenvectors associated with non-positive eigenvalues. After normalizing the remaining eigenvectors, only consider those eigenvectors, which are associated with the smallest k-1 eigenvalues.

Step 9: Allocation of non-generator buses to generator groups using the k-medoids algorithm on the matrix consists of $\mathrm{k} 1$ eigenvectors. The opening of all circuit breakers installed on lines whose terminal buses are in distinct groups will eventually form the desired islands.

Next, each step of the algorithm is explained in detail.

\subsubsection{Dynamic Time Warping (DTW) based Generator Coherency Identification}

Generator coherency identification is primarily a similarity matching problem. Therefore, a method from pattern recognition field can be employed. Several similarity measures have been presented in pattern recognition field including Euclidean distance, Hausdorff, dynamic time warping, Pearson correlation, Mahalanobis, etc. [83], [84]. However, out of these similarity measures, dynamic time warping provides a non-linear mapping between trajectories by minimizing the distance between them [85]. Due to its better performance for partial observability, dynamic time warping was implemented in this chapter for generator coherency determination.

When a disturbance occurs in a power system, the generators' responses are governed by their inertia and location in the system. Some generators exhibit similarity in their time domain responses thus are considered as coherent and can be clustered. Here, rotor angle response is selected as the metric for generator coherence identification. For example, generator $p$ and $q$ are considered coherent if $\Delta \delta p(t)-\Delta \delta q(t) \approx 0$ or $\Delta \delta p(t)-\Delta \delta q(t)=$ constant, where $\Delta \delta p(t)$ and $\Delta \delta p(t)$ are the deviations of rotor angles of generator $p$ and $q$, respectively [89]. In this section, DTW technique is proposed to identify the similarity between rotor angle responses of generators in the system.

Given voltage and current phasor measurements at generator terminal buses, rotor angle responses of these generators can be estimated using least squares (LS) or Kalman filter (KF) based approaches [90]. Consider two rotor angle trajectories $\delta_{p}=\left\{\delta_{p 1}, \delta_{p 2}, \delta_{p 3}, \ldots, \delta p i\right\}$ and $\delta_{q}=\left\{\delta_{q 1}, \delta_{q 2}, \delta_{q 3}, \ldots, \delta q k\right\}$ estimated over the same period, where $i$ and $k$ are numbers of data points for generators $p$ and $q$, respectively. Normally $i$ and $k$ are equal. When there is data loss or significant communication delays in PMU data transmission, $i$ and $k$ may be different, but DTW can still handle the data.

A local distance measure $d\left(\delta_{p m}, \delta_{q n}\right)$ of data points $m$ and $n$ from rotor angle trajectories $\delta_{p}$ and $\delta_{q}$ respectively is defined as: 


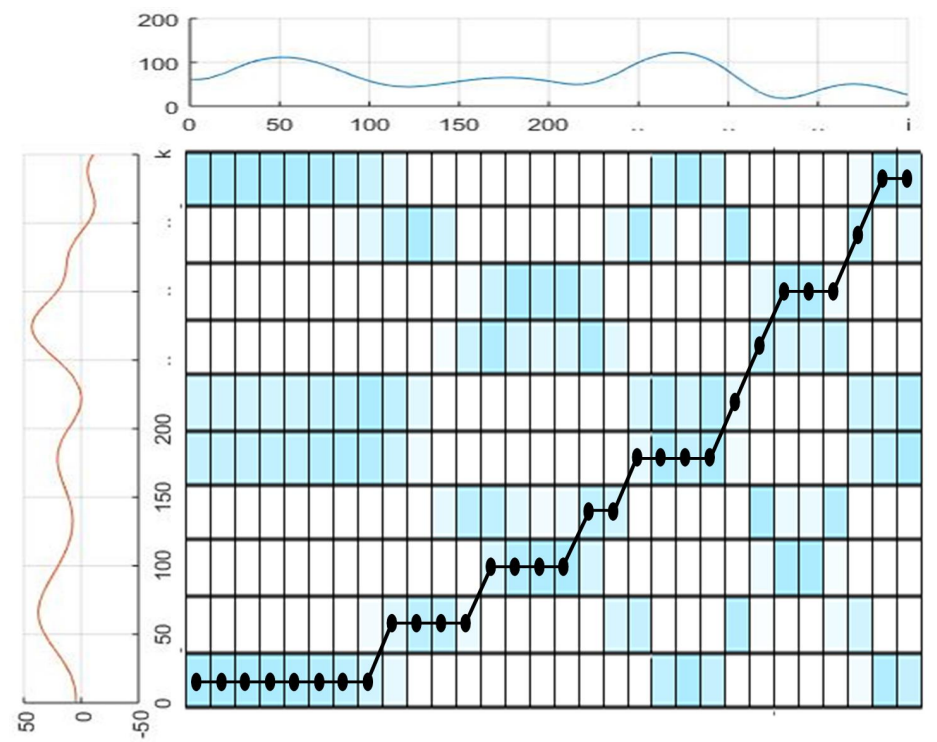

Fig. 3.2 Optimal warping path

$$
d\left(\delta_{p m}, \delta_{q n}\right)=\left\|\delta_{p m}-\delta_{q n}\right\|^{2}
$$

where $m \in\{1,2,3, \ldots, i\}$ and $n \in\{1,2,3, \ldots, k\}$. Similarly, a distance matrix $D\left(\delta_{p}, \delta_{q}\right)$ of size i-by-k is constructed by calculating local distance measures of each pair of data points from trajectories $\delta_{p}$ and $\delta_{q}$.

Define $w=\left\{w_{1}, w_{2}, w_{3}, \ldots, w_{L}\right\}$ as a warping path, where $w_{l}=\left(m_{l}, n_{l}\right) \in[1: i] \times$ $[1: k]$ represents the cell in the $m_{l}$ th row, $n_{l}$ th column of a distance matrix $D\left(\delta_{p}, \delta_{q}\right)$. A valid warping path as shown in Fig. 3 satisfies the following conditions as stated in [91].

1. Boundary condition: a valid warping path starts from one corner of the distance matrix $D\left(\delta_{p}, \delta_{q}\right)$ and ends at the diagonally opposite corner, i.e., $w_{1}=(1,1)$ and $w_{L}=(i, k)$.

2. Continuity: a valid warping path is made of adjacent cells (including diagonally adjacent cells), i.e., $w_{1}=(a, b)$ and $w_{l-1}=\left(a^{\prime}, b^{\prime}\right), a-a^{\prime} \leq 1$ and $b-b^{\prime} \leq 1$.

3. Monotonicity: a warping path is monotone, i.e., if $w_{l}=(a, b)$ and $w_{l}-1=$ $\left(a^{\prime}, b^{\prime}\right), a-a^{\prime} \geq 0$ and $b-b^{\prime} \geq 0$.

The total distance $d_{w}\left(\delta_{p}, \delta_{q}\right)$ of a warping path $w$ is defined as:

$$
d_{w}\left(\delta_{p}, \delta_{q}\right)=\sum_{l=1}^{L} d\left(\delta_{p m l}, \delta_{q n l}\right)
$$

The DTW distance between two trajectories $\delta_{p}$ and $\delta_{q}$ is defined as the minimum total 
distance among all possible warping paths, which can be found by dynamic programming [91].

$$
\operatorname{DTW}\left(\delta_{p}, \delta_{q}\right)=d_{w *}\left(\delta_{p}, \delta_{q}\right)=\min \left\{d_{w}\left(\delta_{p}, \delta_{q}\right)\right\}
$$

In this chapter, the similarity between rotor angle responses of generators $p$ and $q$ is represented by $\operatorname{DTW}\left(\delta_{p}, \delta_{q}\right)$. This allows a non-linear mapping between two trajectories, even with data loss or communication delays. DTW is highly ranked in pattern recognition and computer vision fields. It has been widely used in time series analysis, (partial) shape matching, speech recognition, and online signature verification [92]. In [93], [94], DTW is tested against Euclidean distance for small data size and is found to provide smaller out-of-sample error rate as a result of its improved similarity metric. A comprehensive explanation of step by step implementation of DTW algorithm has been presented with an example in the Appendix A.

Given the coherency of generators, the optimal number of coherent groups $\mathrm{k}$ is selected by minimizing inter-coherent group distances [88]. Further, having the number of coherent groups of generators, various clustering methods, such as k-means clustering [95], can be employed to group generators.

\subsubsection{Buses clustering for controlled islanding}

After clustering generators, the next step is to find an optimal cut set for controlled islanding with generator coherency information as a constraint. The main task is to allocate non-generator buses to coherent generator groups based on a certain metric, which is minimum power flow disruption in this chapter.

Several techniques are present in literature for this "where to island" problem including graph clustering. In [96], a k-way partitioning algorithm was proposed which partitions the power network into islands by optimizing minimum load generation imbalance. A kernel k-means multi-level technique is presented to create islands based on minimum power flow disruption [97]. Both methods are computationally efficient. However, neither of these two methods considers system's dynamic constraints. Furthermore, neglecting generator dynamic behavior may partition the power network into unstable islands. A particle swarm optimization-based angle modulated algorithm is presented in [98] and utilized minimum load generation imbalance to obtain an islanding solution. Reference [99] presents a Krylov based method to minimize load generation imbalance for islanding. Further, the computational complexity of techniques presented in [98], [99] are reduced by neglecting connectivity of sub-graphs or solving for a simplified network. However, the islanding solutions may contain isolated buses or some solutions, which are lost after simplification, could be better than the solution obtained by the algorithm [100]. Spectral clustering-based approach is used to solve the "where to island" problem in this chapter, which builds on the concept of minimum 
graph-cut [101]. The objective is to minimize the power flow disruption subject to the generator grouping from Section 3.2.1. The minimum graph cut formulation is stated as follows.

Power network can be represented as a weighted graph $G=(V, E, W)$ with vertices $(V)$ and edges $(E)$ resembling buses and branches (lines or transformers), respectively. To replicate characteristics of the power grid, each edge in the graph is assigned a certain weight $(W)$, which can be any system parameter depending on the targeted application. We will use power flows through branches as the weighting factors. Further, to accommodate system losses, weights are evaluated by averaging power flows measured at both sides of the lines as follows.

$$
W_{i j}=W_{j i}= \begin{cases}\left(\left|P_{i j}\right|+\left|P_{j i}\right| / 2\right. & (i \neq j) \\ 0 & (i=j)\end{cases}
$$

where $P_{i j}$ and $P_{j i}$ are the active power flows measured at terminals $i$ and $j$ of branch $i-j$, respectively. The weight matrix in (3.4) considers the dynamic characteristic of power network as power flow changes with system operating conditions. After evaluating the weight matrix, an un-normalized Laplacian matrix, $L$, can be formulated with its element $L_{i j}$ calculated as:

$$
L_{i j}= \begin{cases}-W_{i j} & (i \neq j) \\ d_{i}=\sum_{j=1}^{n} W_{i j} & (i=j)\end{cases}
$$

where $d i$ is the sum of weights of all edges connected to node $i$. To make graphs with different weights comparable, the Laplacian matrix can be normalized as $L_{N}=$ $D^{1 / 2} L D^{1 / 2}$ [102], where $D$ is a diagonal degree matrix with $d_{i}$ as its diagonal entries.

Given the coherent generator groups, we apply spectral clustering to further cluster buses for controlled islanding. To incorporate generator coherency information as a constraint in spectral clustering, two types of linkages can be introduced: must link (ML) and cannot link (CL). ML constraints ensure the coherent generators remain on the same island while CL keeps the non-coherent generators on different islands. A linkage constraint matrix $\mathrm{Q}$ is defined as:

$$
Q_{i j}= \begin{cases}+1 & i, j \in M L \\ -1 & i, j \in C L \\ 0 & \text { else }\end{cases}
$$

Let $u \in\{-1,+1\}^{N}$ be an island indicator vector for $N$ nodes, where $u_{i}=+1$ if bus $i$ belongs to island ' + ' and $u_{i}=-1$ if bus $i$ belongs to island '-'. An index $u^{T} Q u=$ $\sum_{i j} u_{i} u_{j} Q_{i j}$ can be defined to determine how well constraints in $Q$ are satisfied by 
the assignment $u$. The greater the value of $u^{T} Q u$ is, the more satisfied the coherency constraints $Q$ are by the associated indicator vector $u$ [103]. Variables $u_{i}$ and $Q$ can be extended to more than two islands as $u \in R^{N}$ and $Q \in R^{N N}$ respectively. If $Q_{i j}>0$, then buses $i$ and $j$ should be in the same island and if $Q_{i j}<0$ buses $i$ and $j$ should be placed in different islands. Similar to the normalized Laplacian matrix, constraint matrix $Q$ can also be normalized as $Q_{N}=D^{-1 / 2} Q D^{1 / 2}$. Finally, the association of non-generator buses to already identified generator groups can be obtained by solving the following constrained optimization problem [88]:

$$
\begin{cases} & \min _{v} v^{T} L_{N} v \\ \text { s.t } & v^{T} Q_{N} v>\beta \\ & v^{T} v=v o l \\ & v \neq D^{1 / 2} 1\end{cases}
$$

where $v^{T} L_{N} v$ is the cost of the spectral cut; $\beta$ is the satisfaction threshold for constraints; vol $=\sum_{i}^{N} d_{i}$ is the volume measure of the graph. $v^{T} v=v o l$ is used to normalize $v$ and $v \neq D^{1 / 2} 1$ is used to avoid trivial solutions with $\mathbf{1}$ as a constant vector whose entries are 1's. The relaxed island indicator vector $u$ can be recovered from $v$ as $u=D^{1 / 2} v$. The optimal solution of (3.9) can be obtained using Karush-Kuhn-Tucker theorem [104] by solving the following generalized eigenvalue problem:

$$
L_{N} v=\lambda\left(Q_{N}-\frac{\beta}{v o l} I\right) v
$$

After normalizing eigenvectors associated with positive eigenvalues using $\mathbf{v} \leftarrow$ $\frac{v}{\|v\|} \sqrt{v o l}$ and $k$ being the coherent generator groups obtained through the proposed active nodes clustering , $k-1$ eigenvectors with lowest eigenvalues are selected. Finally, the k-medoids algorithm [105] can be applied, on a matrix $V^{*}$ having $k-1$ eigenvectors as columns. It will allocate non-generator buses to $k$ islands. The proposed adaptive controlled islanding scheme is shown in Fig. 3.3.

\subsubsection{Discussion}

The main scope of this work is to present an approach, which can find more stable islands. An islanding solution based on constrained spectral clustering is proposed which splits the power network into islands using minimum power flow disruption. Additionally, to ensure the dynamic stability of the newly formed islands, DTW based generator coherency information is treated as a constraint during spectral clustering based splitting process [106].

Finding an islanding solution with minimum load generation imbalance is indeed an NP-hard problem and considered as a special form of 0-1 knapsack problem. This is 


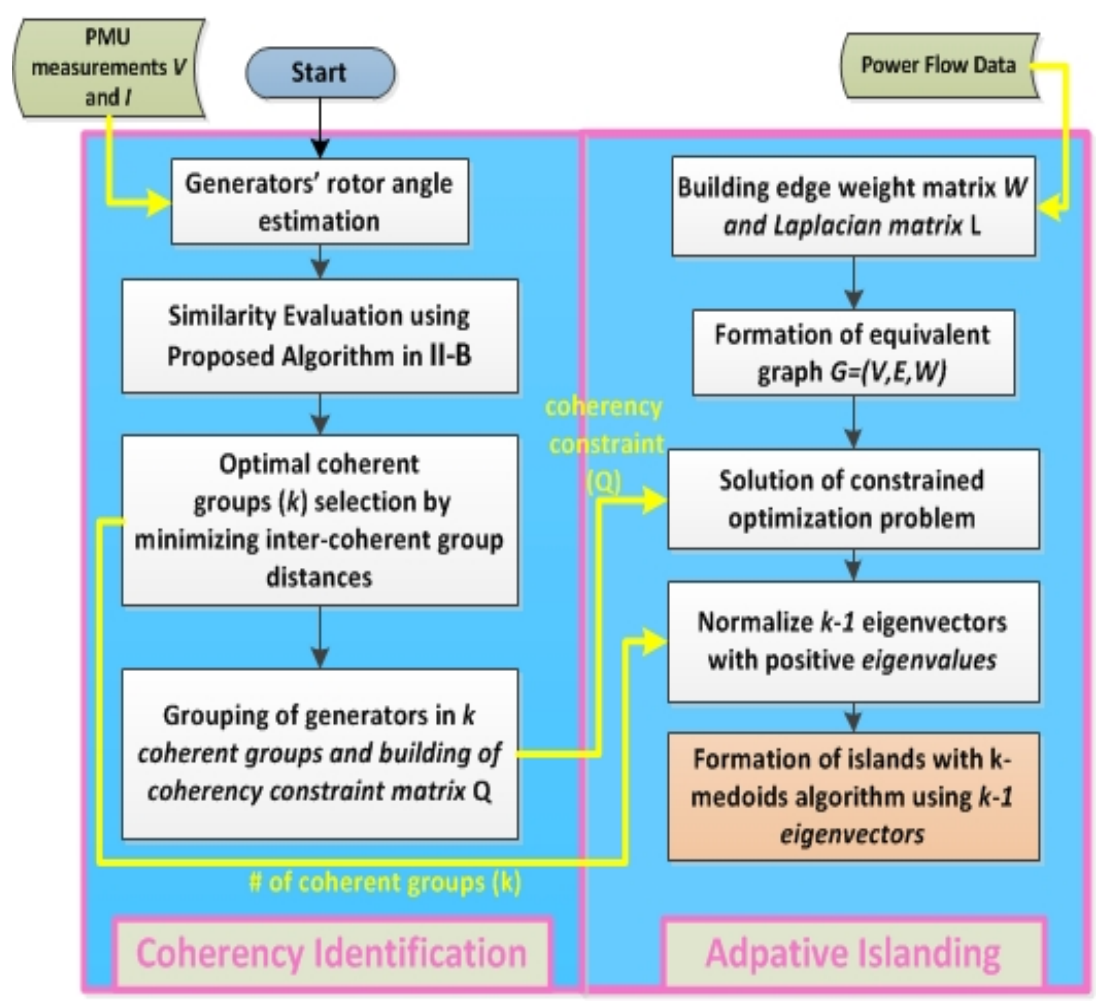

Fig. 3.3 Algorithm 1 (adaptive controlled islanding)

why in this work we considered minimum power flow disruption instead of minimum load generation imbalance as the criterion for spectral clustering. It is a P-problem as can be converted into a minimum-cut problem and hence solved efficiently [107]. Consideration of generator coherency constraints during spectral clustering increases its complexity. However, this increased computational complexity can be overcome using recursive bisection to find island boundaries [108]. Thus, using minimum power flow disruption has the advantage of reducing the time complexity from NP-hard to P that makes the proposed DTW based islanding approach computationally efficient.

\subsection{Simulation Results and Performance Evaluation}

The proposed methodology is validated through dynamic simulations of IEEE 39-bus and WECC-179 systems. Cascading outages are created using DSAT tools. Time domain simulations show how the proposed methodology can help in minimizing the impact of cascading outages and avoiding blackouts.

Proposed DTW based coherency is determined using $t=5-7.5 \mathrm{~s}$ of data and is utilized further during constrained spectral clustering. The islanding scheme will then be available in case it is needed. The severity and stability indices during cascading failure will determine the exact timing of the islanding. In the experimental validation, islanding was deployed at $\mathrm{t}=9 \mathrm{~s}$ to realize the practical situation where closed-loop algorithms may try to bring the system back towards stability and if those algorithms fail then as a 
last resort islanding can be adopted to avoid further failure. There is no other reason for the islanding time selection of $\mathrm{t}=9 \mathrm{~s}$.

Length of the PMU data is critical for the identification results due to cascading failures occurring at different instances as reported in [71]. We do not attempt to duplicate that discussion but rather emphasize the particular advantages DTW has that makes it suitable for applications in generator coherence identification and controlled islanding.

The simulation is performed on a 64-bit Intel $(\mathbb{R}$ Core i7 Central Processing Unit with 3.00 gigahertz speed, 12 gigabytes installed memory (RAM) and 1 terra byte hard disk space. For both case studies, running the proposed algorithm on Matlab takes 1.12 s. It is expected running the same program using c/c++ will be 50-100 times faster, which satisfies the online application requirements.

Transient stability essentially means that the generators in one island should maintain synchronization after islanding operation. Transient stability of the newly formed islands primarily depends on the coherency of the generators. Silhouette measure can be used to validate the coherent group formation identified by coherency determination algorithms, which is a measure to validate the consistency of the clusters in the data [109], [110]. Utilizing this index, it can be shown that how strongly generators in one group are coherent and coupled together as compared to those in other coherent groups. It is expressed as:

$$
V_{i}^{S}= \begin{cases}{\left[S_{\text {min,avg }}^{*}(i)-S_{\text {avg }}^{*}(i)\right] / S_{\text {min }, a v g}^{*}(i)} & \text { if } S_{\text {avg }}^{*}(i)<S_{\text {min }, a v g}^{*}(i) \\ 0 & \text { if } S_{\text {avg }}^{*}(i)=S_{\text {min }, \text { avg }}^{*}(i) \\ {\left[S_{\text {min,avg }}^{*}(i)-S_{\text {avg }}^{*}(i)\right] / S_{\text {avg }}^{*}(i)} & \text { if } S_{\text {avg }}^{*}(i)>S_{\text {min }, a v g}^{*}(i)\end{cases}
$$

where $S_{m i n, a v g}^{*}(i)$ is the minimum average dissimilarity of $i$ th generator w.r.t other coherent groups, and $S_{\text {avg }}^{*}(i)$ is the average dissimilarity of $i$ th generator w.r.t all other generators in the same coherent group. A generator with large silhouette value shows that it is strongly coupled with the generators of its coherent group and weakly coupled with the neighboring groups. Typically, silhouette value lies between 1 and -1 . Generators coherency identification is appropriate if most of the generators have large silhouette values. On the other hand, if silhouette values are very small or negative, it shows the possibility of too many coherent groups [111]. The criteria to determine the most suitable coherent group formation is to check the average silhouette value. The coherent groups' formation having higher average silhouette value will indicate more strongly coupled coherent groups [111].

The coherent generator groups' formation with maximum average silhouette value is considered more appropriate coherency identification [112]. Hence, more coherent generator groups formation will surely ensure more transient stability after islanding 
and will help in healing the system and avoiding a further blackout. A few researchers have recently used this coherency validation criterion for coherent generator groups' formation evaluation. Therefore, it can be regarded as a measure of the stability of the newly formed islands [111], [112].

We compared stability in the form of silhouette measure, which is independent of the number of clusters/islands. Also, since the system is already recovering from cascading failures, maintaining transient stability is more crucial and focused in this work than maintaining load generation balance. Load generation imbalance is a bi-product and shows an additional benefit that the proposed approach is carrying. Moreover, researchers have shown that loss of load is not directly related to the number of islands formed [106], [111], [112], [113]. The comparisons in [106], [112], [113] indicate that the loss of load is more for less number of islands formed and amount of load lost is not directly proportional to the number of islands.

To compare the performance of the proposed approach with existing benchmarks, same "where to island" algorithm, i.e., spectral clustering is used to find the boundaries of the islands; whereas, generator coherency information is determined through DTW, correlation and community detection methods. Islanding solutions are compared regarding stability, minimum load generation imbalance and minimum power flow disruption. The coherent group formation with maximum average silhouette value ensures more stability, serving as the primary objective for controlled islanding and also helps to avoid further cascading failures.

\subsubsection{Case Study 1: Comparison with Correlation based Method}

In this case, a 3-phase fault is applied on line 17-16 near bus 17 at $\mathrm{t}=5 \mathrm{~s}$ and cleared after $150 \mathrm{~ms}$ with the tripping of the corresponding line. Another line 2-1 is tripped at $\mathrm{t}=7 \mathrm{~s}$ following a 3 -phase fault of $280 \mathrm{~ms}$ duration [114]. These cascading outages eventually lead the system to lose synchronism at $\mathrm{t}=12.36 \mathrm{~s}$ as shown in Fig. 3.4.a. Voltage magnitudes at buses also go very low resulting in a blackout as can be seen in Fig. 3.4.b.

The loss of synchronism and voltage violations are clear indications that the system should be split. In a practical implementation, the timing of splitting is determined by the system operator. Moreover, it depends on the vulnerability analysis performed after

Table 3.1 Allocation of non-generator buses

\begin{tabular}{|ll|}
\hline Island 1 & Island 2 \\
\hline \hline $2,3,17,18,25,26,27,28,29,30$, & $1,4,5,6,7,8,9,10,11,12$, \\
37,38 & $13,14,15,16,19,20,21,22,23,24$ \\
& $31,32,33,34,35,36,39$ \\
\hline
\end{tabular}




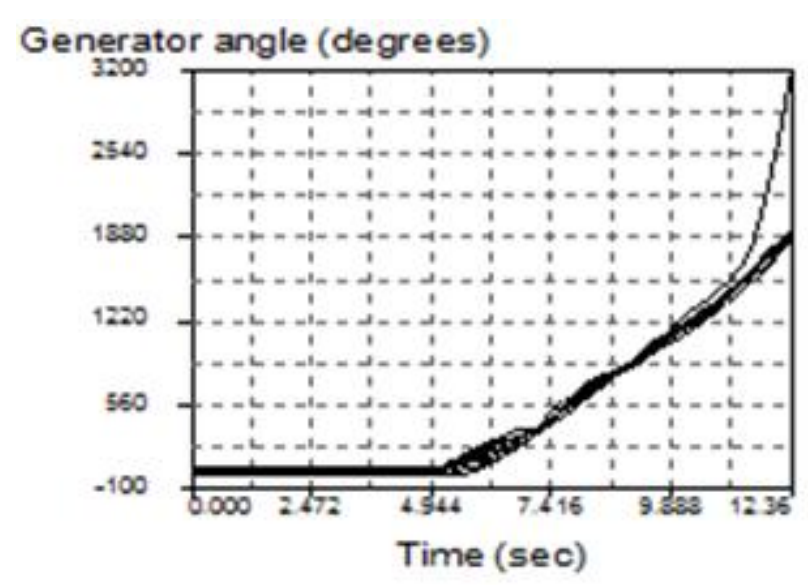

(a)

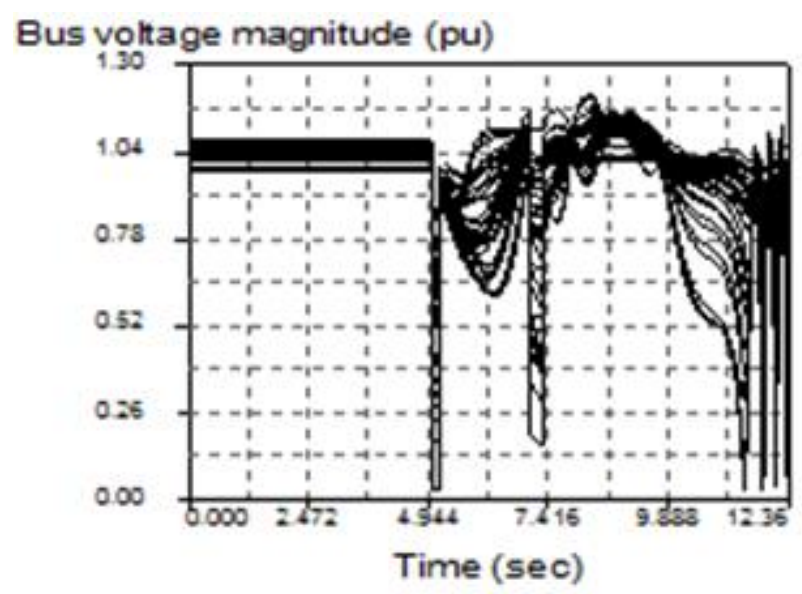

(b)

Fig. 3.4 System losing synchronism and becoming unstable

severe disturbances [102]. In this chapter, we implement intentional islanding at $t=9 \mathrm{~s}$ following two cascading outages. The proposed approach provides a suitable islanding solution using online coherency and pre-fault power flow conditions. The proposed generators coherency algorithm identifies two coherent generator groups as (G1, G8, G9) and (G2, G3, G4, G5, G6, G7). We use this information and solve a constrained spectral clustering problem as described in Section 3.2.2. Table 3.1 shows the allocation of non-generator buses to coherent generator groups. It suggests that the breaker on line 3-4 should be opened to form two islands as shown in Fig. 3.5, and 74.76 MW of active power is disrupted.

Generators rotor angles also show the clear formation of two coherent groups after islanding as shown in Fig. 3.6.a. Voltage magnitude at buses is within limits as can be seen in Fig. 3.6.b. The numerical results suggest that Algorithm 1 is capable of avoiding system-wide blackouts by keeping voltages at buses within limits and maintaining generators synchronism.

In this case, G10 can be considered as a separate island [106] or as a reference [72], 


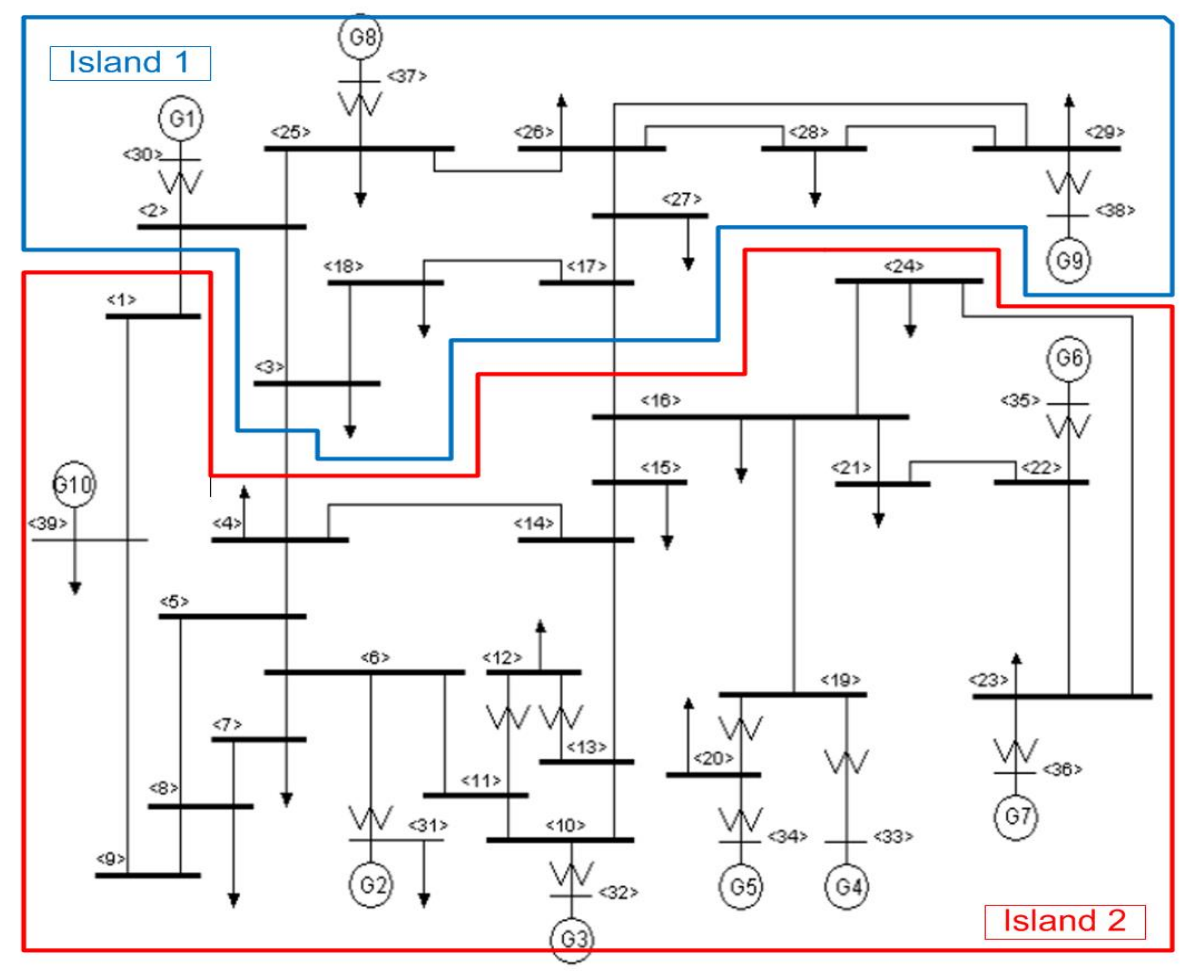

Fig. 3.5 Formation of two islands

[115] during coherency identification. We adopted the latter approach in this dissertation. That is why it is not directly considered for DTW based coherency determination. However, its inclusion in the final islanding solution depends on its location and minimum power flow disruption based spectral clustering results.

To check the quality of islanding, active and reactive power generation capacities and load demands are evaluated for each independent island as presented in Table 3.2. Generators in each island are capable of fulfilling local demand after islanding. Hence, the proposed online coherency algorithm is capable of identifying suitable generator groups which can be used as dynamic constraints for intentional islanding at the expense

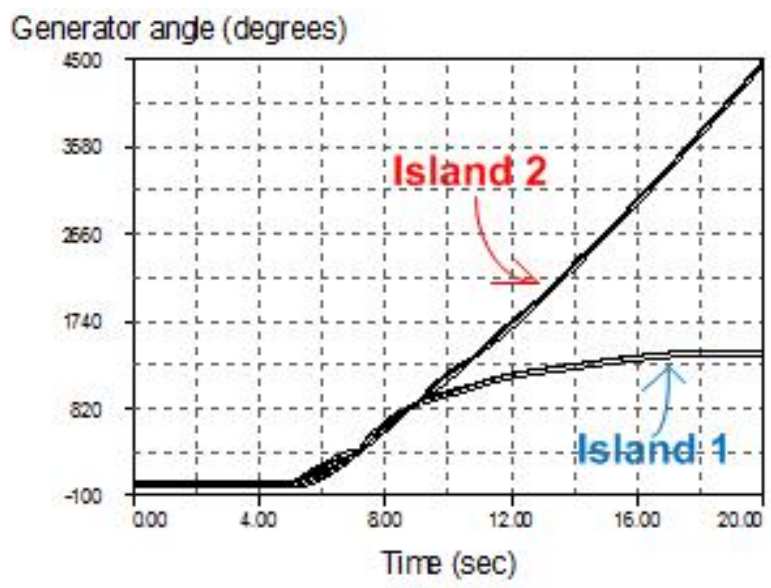

(a) 


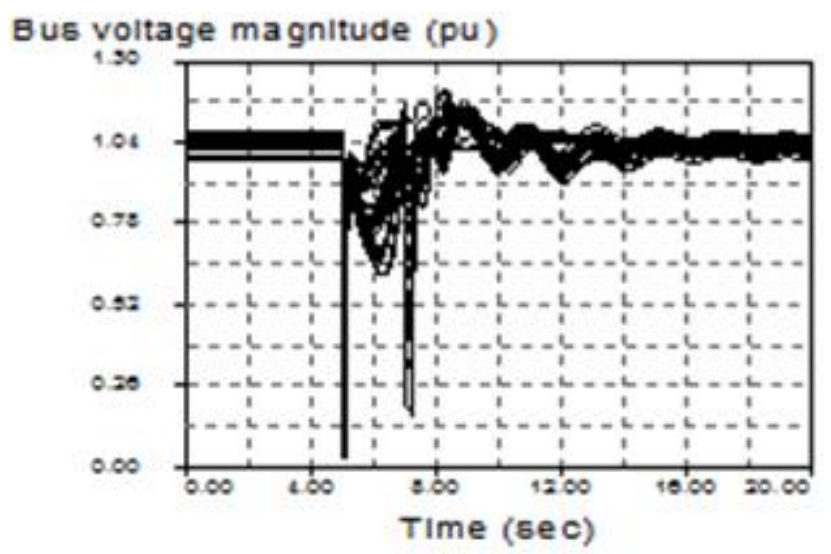

(b)

Fig. 3.6 Generators rotor angle responses and voltage profiles at system buses after implementing proposed islanding scheme

of no load shed to avoid a blackout.

The correlation-based method proposed in [114] is carried out as a benchmark. It calculates the correlation coefficient for each pair of generators and splits them based on the average correlation value.

The correlation-based method identifies three coherent groups as (G2, G3), (G4, G5, G6, G7), (G1, G8, G9). The generation capacity of island 1 is below the local demand of the island as shown in Table 3.3. About 145.1 MW load is shed as shown in Fig. 3.7.b with the red color area at the top of the load bar. Further, as shown in Fig. 3.8 , the proposed DTW based approach shows a higher value of the stability measure, hence indicates better tightness for the coherent generators group formation and will be more transiently stable.

Moreover, breakers on lines 3-4 and 14-15 are opened to split the system into three islands. On the other hand, Algorithm 1 sheds no loads with fewer islands and breaker operations. A complete comparison of Algorithm 1 and correlation method based islanding is in Table 3.4.

Table 3.2 Active and reactive power balances in each island using proposed approach

\begin{tabular}{|l|l|l|l|l|}
\hline $\begin{array}{l}\text { Is- } \\
\text { land }\end{array}$ & $\begin{array}{l}\text { Active power } \\
\text { generation } \\
\text { capacity } P_{G} \\
\text { (p.u) }\end{array}$ & $\begin{array}{l}\text { Active power } \\
\text { load demand } \\
P_{L} \text { (p.u) }\end{array}$ & $\begin{array}{l}\text { Reactive } \\
\text { power } \\
\text { generation } \\
\text { capacity } Q_{G} \\
\text { (p.u) }\end{array}$ & $\begin{array}{l}\text { Reactive } \\
\text { power load } \\
\text { demand } Q_{L} \\
\text { (p.u) }\end{array}$ \\
\hline \hline 1 & 16.20 & 16.13 & $\begin{array}{l}+24 \text { to }-15 \\
+59 \text { to }-38\end{array}$ & 3.266 \\
2 & 45.73 & 45.36 & 14.73 \\
\hline
\end{tabular}


Table 3.3 Active and reactive power balances in each island using correlation-based algorithm

\begin{tabular}{|l|l|l|l|l|}
\hline $\begin{array}{l}\text { Is- } \\
\text { land }\end{array}$ & $\begin{array}{l}\text { Active power } \\
\text { generation } \\
\text { capacity } P_{G} \\
\text { (p.u) }\end{array}$ & $\begin{array}{l}\text { Active power } \\
\text { load demand } \\
P_{L} \text { (p.u) }\end{array}$ & $\begin{array}{l}\text { Reactive } \\
\text { power } \\
\text { generation } \\
\text { capacity } Q_{G} \\
\text { (p.u) }\end{array}$ & $\begin{array}{l}\text { Reactive } \\
\text { power load } \\
\text { demand } Q_{L} \\
\text { (p.u) }\end{array}$ \\
\hline \hline 1 & 22.239 & 23.69 & +31 to -20 & 7.866 \\
2 & 23.50 & 21.595 & +28 to -18 & 6.858 \\
3 & 16.20 & 16.13 & +24 to -15 & 3.266 \\
\hline
\end{tabular}

\subsubsection{Case Study 2: Comparison with Community detection method}

A 3-phase fault is simulated on line 13-14 near bus 13 at $\mathrm{t}=5 \mathrm{~s}$ and cleared after 150 ms with the tripping of the line. Another 3-phase fault of 6 cycles duration is simulated in the middle of the line 16-17 at $\mathrm{t}=7 \mathrm{~s}$ [116]. Following these cascading outages, the system loses synchronism at $\mathrm{t}=11.45 \mathrm{~s}$, and voltage magnitudes also go beyond permissible limits as shown in Fig. 3.9.a and Fig. 3.9.b respectively if the islanding is not implemented.

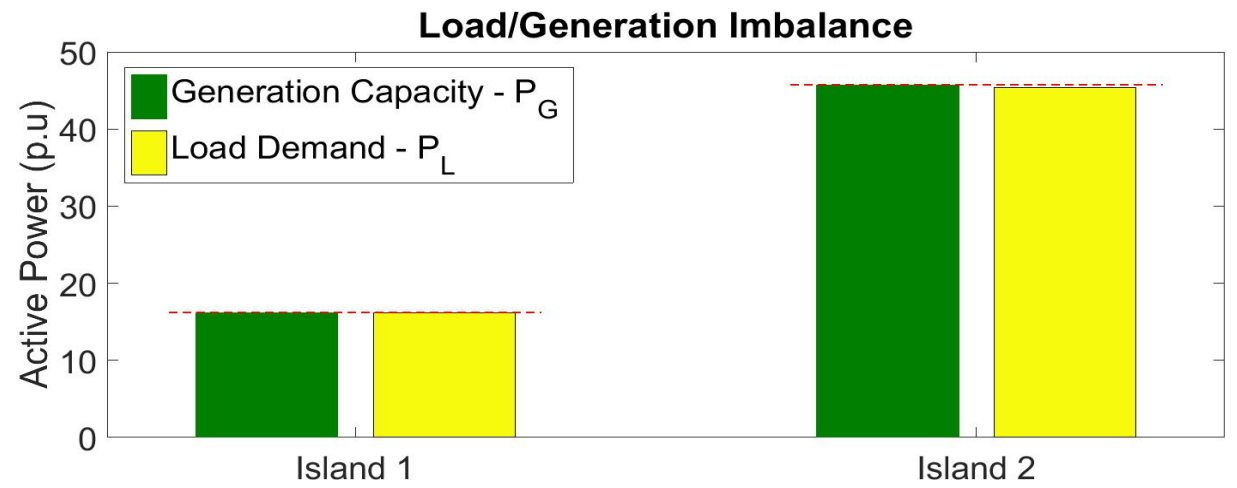

(a) Proposed method

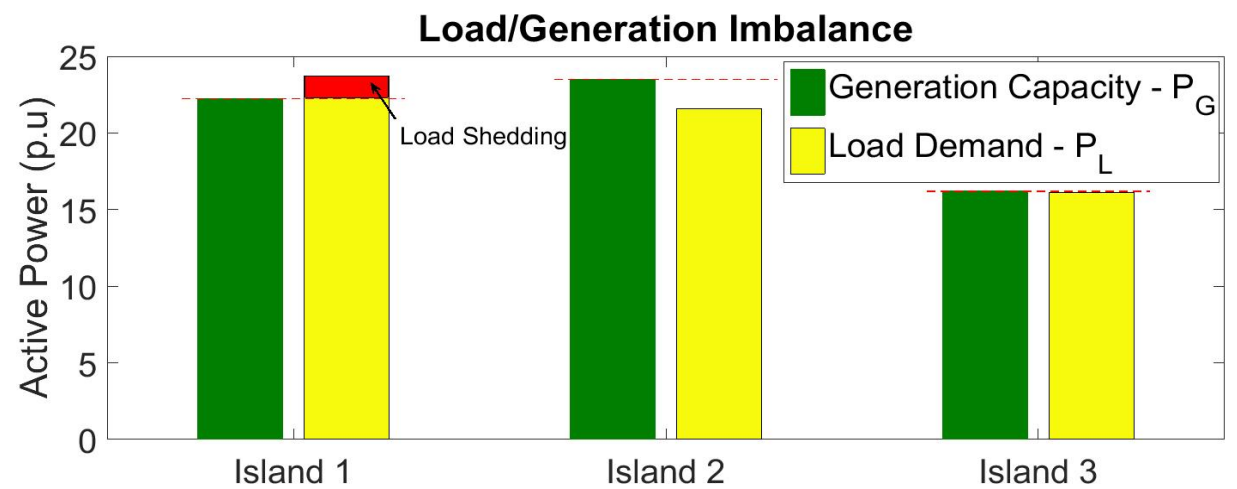

(b) Correlation-based method

Fig. 3.7 Active power load shedding comparison 


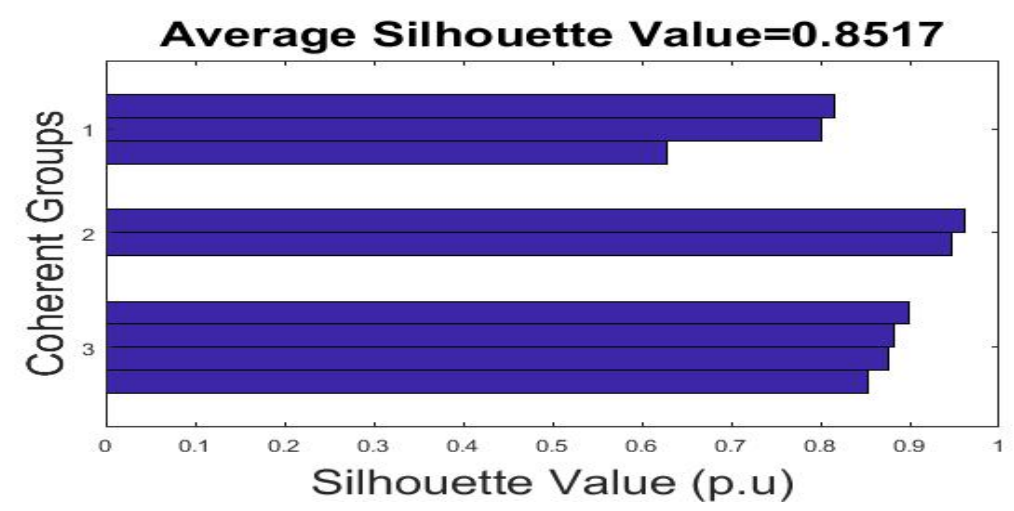

(a) Correlation-based method

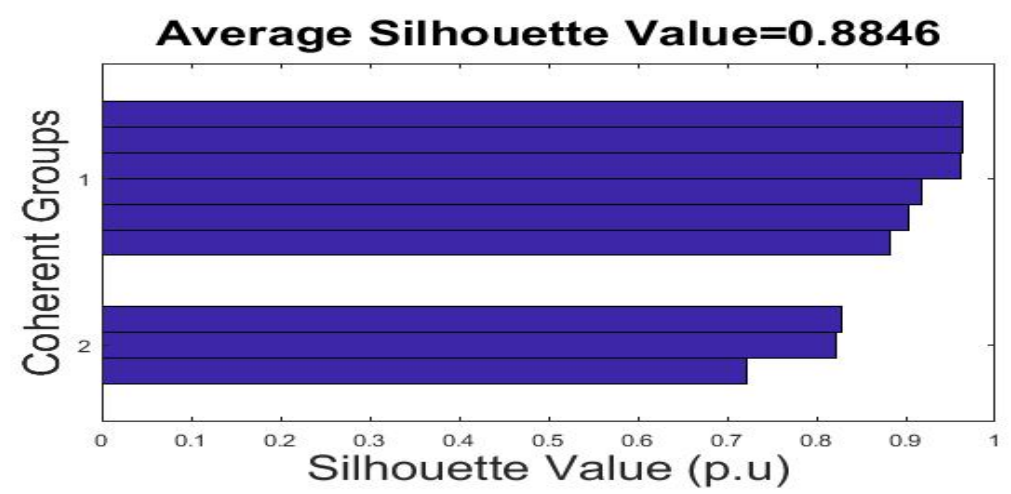

(b) Proposed method

Fig. 3.8 Silhouette plots for coherent generators groups

The proposed coherency algorithm identifies two generator groups as (G1, G2, G3, G8, G9) and (G4, G5, G6, G7). Solving the constrained spectral clustering problem, we get the allocation of non-generator buses as presented in Table 3.5. According to the allocation, the breaker on line 14-15 should be opened to split the system into two islands as shown in Fig. 3.10. 33.41 MW power is disrupted. Rotor angle trajectories

Table 3.4 Performance comparison between proposal and correlation-based algorithms

\begin{tabular}{|l|l|l|l|l|l|}
\hline Method & $\begin{array}{l}\text { No. of } \\
\text { switching } \\
\text { operations }\end{array}$ & $\begin{array}{l}\text { Power flow } \\
\text { disrup- } \\
\text { tions } \\
\text { (MW) }\end{array}$ & $\begin{array}{l}\text { Load/ } \\
\text { generation } \\
\text { imbalance } \\
\text { (MW) }\end{array}$ & $\begin{array}{l}\text { Load } \\
\text { shed } \\
\text { (MW) }\end{array}$ & $\begin{array}{l}\text { Silhouette } \\
\text { value }\end{array}$ \\
\hline $\begin{array}{l}\text { Proposed } \\
\text { algorithm } \\
\text { based } \\
\text { islanding }\end{array}$ & 1 & 74.76 & 44.27 & 0 & 0.8846 \\
\hline $\begin{array}{l}\text { Correlation } \\
\text { coefficient } \\
\text { based } \\
\text { islanding }\end{array}$ & 2 & 108.17 & 342.6 & 145.1 & 0.8517 \\
\hline
\end{tabular}




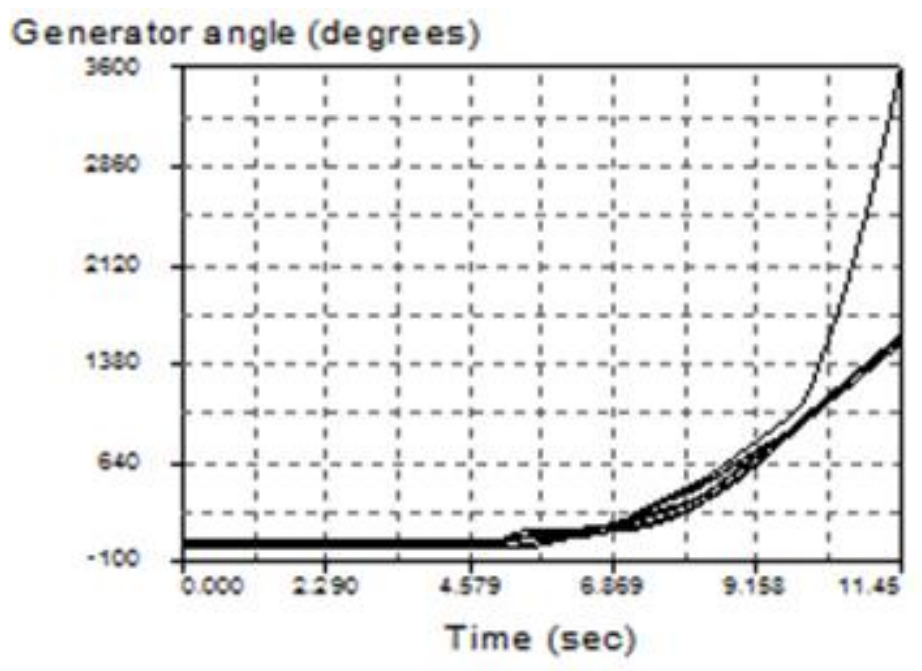

(a)

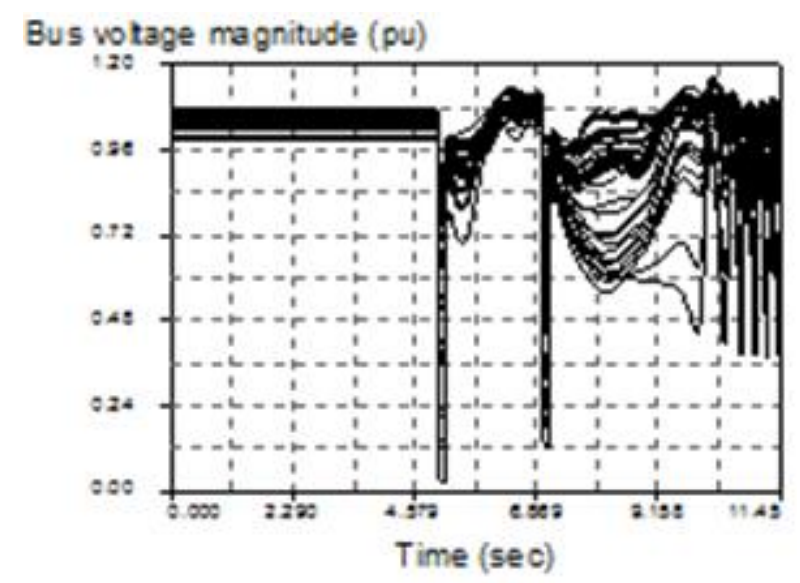

(b)

Fig. 3.9 System losing synchronism and becoming unstable

shown in Fig. 3.11.a indicate the synchronism of generators after islanding. Voltage magnitudes are also within limits as shown in Fig. 3.11.b.

Active and reactive power generation/load imbalance is evaluated for each island as shown in Table 3.6. Generators in island 1 are capable of fulfilling the load demand. However, 137.7 MW load is shed in island 2 for stable and balanced operation as shown in Fig. 3.12.a.

We also carry out community detection method introduced in [116], and results are summarized in Table 3.7. Community detection method identifies three coherent

Table 3.5 Allocation of non-generator buses

\begin{tabular}{|ll|}
\hline Island 1 & Island 2 \\
\hline \hline $15,16,19,20,21,22,23,24,33$, & $1,4,5,6,7,8,9,10,11,12$, \\
$34,35,36$ & $13,14,15,16,19,20,21,22$, \\
& $23,24,31,32,33,34,35,36,39$ \\
\hline
\end{tabular}




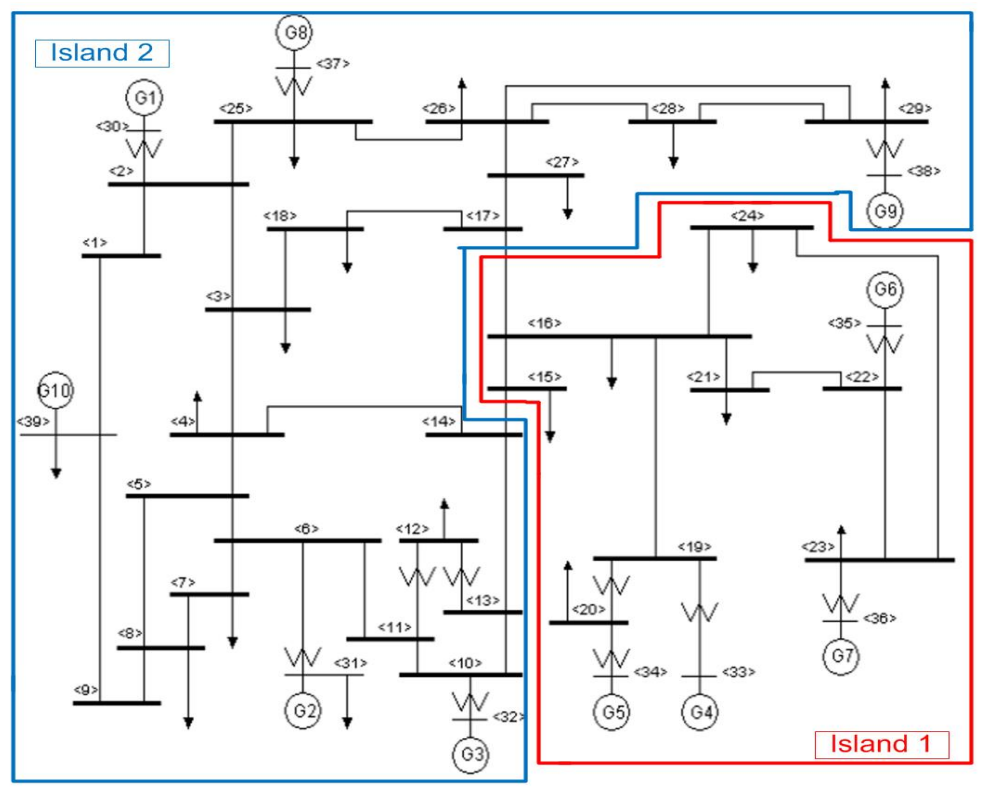

Fig. 3.10 Formation of two islands

generator groups as $(\mathrm{G} 2, \mathrm{G} 3),(\mathrm{G} 4, \mathrm{G} 5, \mathrm{G} 6, \mathrm{G} 7),(\mathrm{G} 1, \mathrm{G} 8, \mathrm{G} 9)$. The active power generation capacities of island 1 and island 3 are less than the demand of each island. As a consequence, 50.6 MW and 96.43 MW loads are shed in island 1 and 3 respectively as shown in Fig. 3.11.b. As seen in Fig. 3.13, the proposed DTW based approach shows a higher value of the stability measure, hence will be more transiently stable. Breakers on lines 3-4, 8-9 and 14-15 are opened to split the system into three islands. A complete comparison of Algorithm 1 and community detection method based islanding can be seen in Table 3.8, which also indicates superior performance of the proposed algorithm.

To obtain appropriate islanding, load generation imbalance is not as crucial and critical as ensuring the transient stability within islands as the system is already recovering from cascading outages. Moreover, in an island, load generation imbalance can be compensated through partial load shedding. However, an island with negative stability

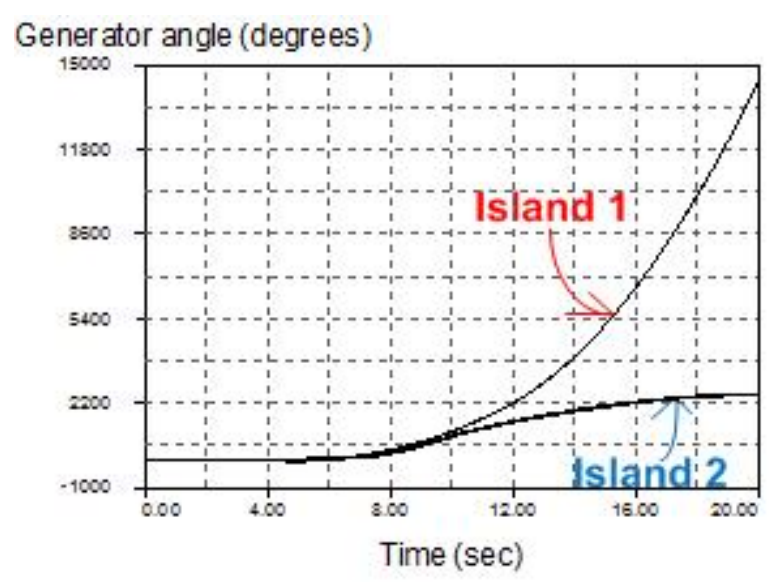

(a) 


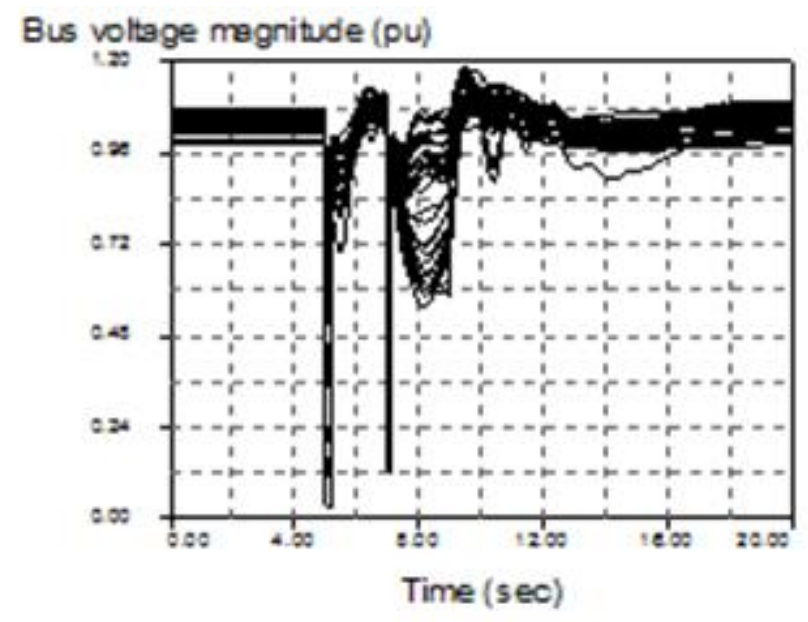

(b)

Fig. 3.11 Generators rotor angle responses and voltage profiles at system buses after proposed islanding

margin and good load generation imbalance will collapse. Hence, a proper islanding solution must ensure that coherent generators remain on the same island to improve the stability and reduce the chances of further outages [106].

DTW based coherency determination can still handle the data even if the compared

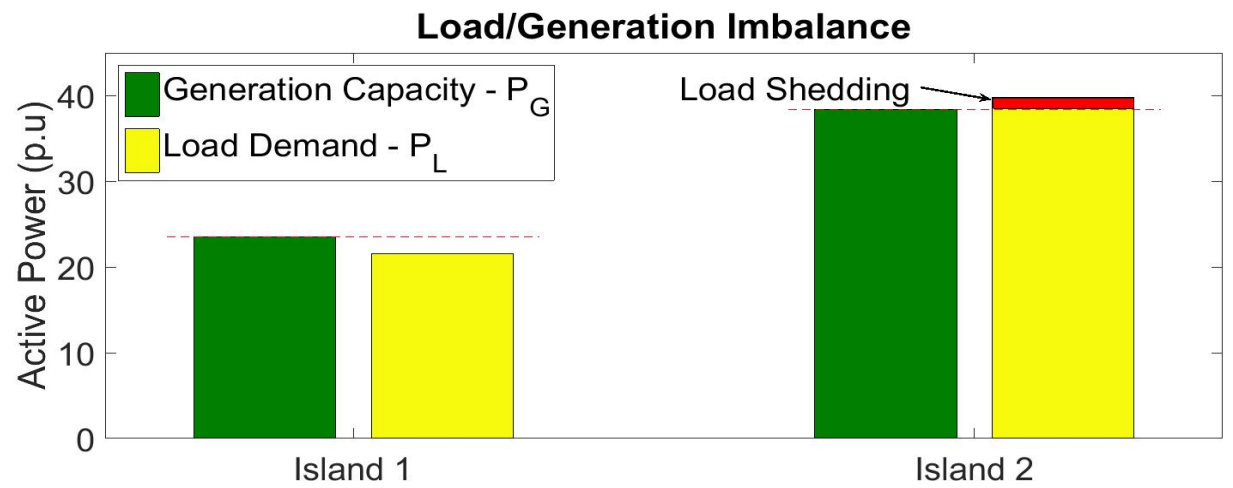

(a) Proposed method

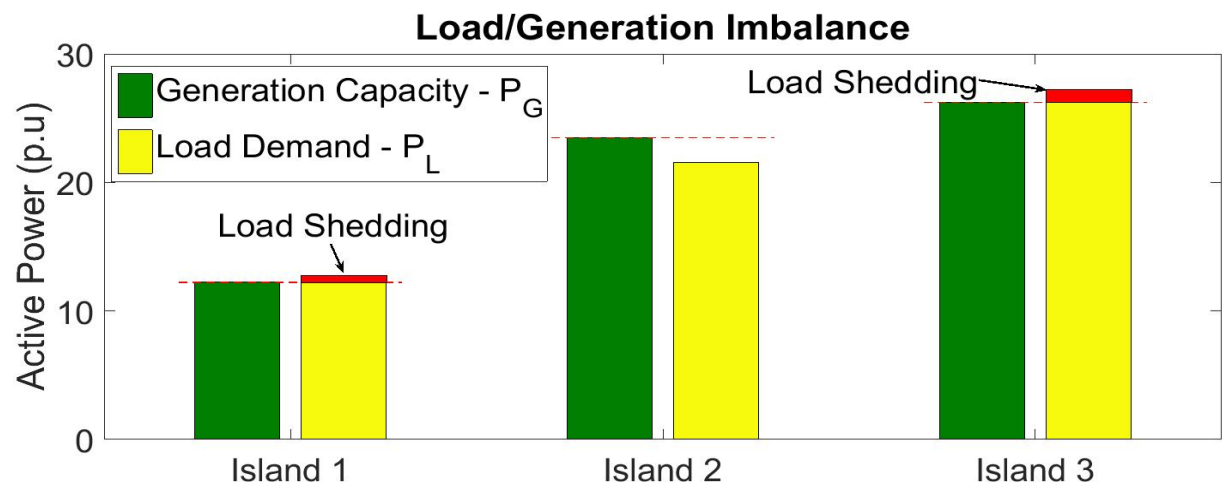

(b) Community detection based method

Fig. 3.12 Active power load shedding comparison 
Table 3.6 Active and reactive power balances in each island using proposed approach

\begin{tabular}{|c|c|c|c|c|}
\hline $\begin{array}{l}\text { Is- } \\
\text { land }\end{array}$ & $\begin{array}{l}\text { Active power } \\
\text { generation } \\
\text { capacity } P_{G} \\
\text { (p.u) }\end{array}$ & $\begin{array}{l}\text { Active power } \\
\text { load demand } \\
P_{L} \text { (p.u) }\end{array}$ & $\begin{array}{l}\text { Reactive } \\
\text { power } \\
\text { generation } \\
\text { capacity } Q_{G} \\
\text { (p.u) }\end{array}$ & $\begin{array}{l}\text { Reactive } \\
\text { power load } \\
\text { demand } Q_{L} \\
\text { (p.u) }\end{array}$ \\
\hline $\begin{array}{l}1 \\
2\end{array}$ & $\begin{array}{l}23.50 \\
38.43\end{array}$ & $\begin{array}{l}21.59 \\
39.81\end{array}$ & $\begin{array}{l}+28 \text { to }-18 \\
+55 \text { to }-35\end{array}$ & $\begin{array}{l}7.16 \\
10.83\end{array}$ \\
\hline
\end{tabular}

Table 3.7 Active and reactive power balances in each island using community detection based algorithm

\begin{tabular}{|l|l|l|l|l|}
\hline $\begin{array}{l}\text { Is- } \\
\text { land }\end{array}$ & $\begin{array}{l}\text { Active power } \\
\text { generation } \\
\text { capacity } P_{G} \\
\text { (p.u) }\end{array}$ & $\begin{array}{l}\text { Active power } \\
\text { load demand } \\
P_{L} \text { (p.u) }\end{array}$ & $\begin{array}{l}\text { Reactive } \\
\text { power } \\
\text { generation } \\
\text { capacity } Q_{G} \\
\text { (p.u) }\end{array}$ & $\begin{array}{l}\text { Reactive } \\
\text { power load } \\
\text { demand } Q_{L} \\
(\mathbf{p . u})\end{array}$ \\
\hline \hline 1 & 12.229 & 12.735 & +16 to -10 & 5.366 \\
2 & 23.50 & 21.58 & +28 to -18 & 6.864 \\
3 & 26.21 & 27.175 & +39 to -25 & 5.766 \\
\hline
\end{tabular}

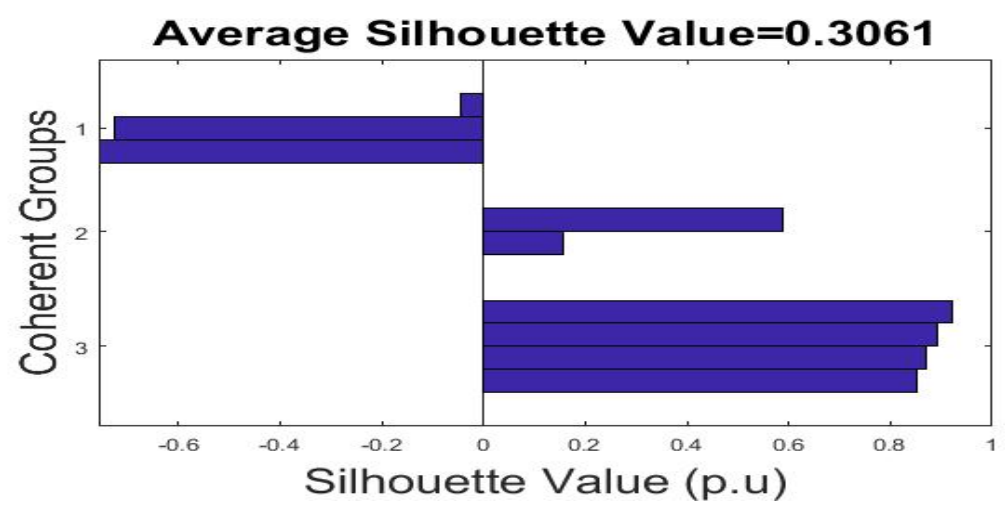

(a) Community detection based method

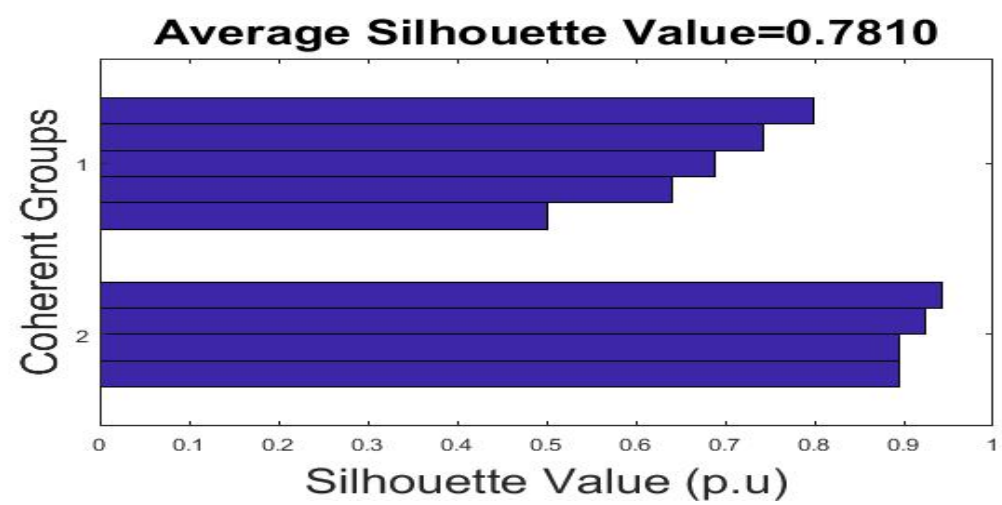

(b) Proposed method

Fig. 3.13 Silhouette plots for coherent generators groups 
Table 3.8 Performance comparison between proposal and community detection based algorithms

\begin{tabular}{|l|l|l|l|l|l|}
\hline Method & $\begin{array}{l}\text { No. of } \\
\text { switching } \\
\text { operations }\end{array}$ & $\begin{array}{l}\text { Power flow } \\
\text { disrup- } \\
\text { tions } \\
\text { (MW) }\end{array}$ & $\begin{array}{l}\text { Load/ } \\
\text { generation } \\
\text { imbalance } \\
\text { (MW) }\end{array}$ & $\begin{array}{l}\text { Load } \\
\text { shed } \\
\text { (MW) }\end{array}$ & $\begin{array}{l}\text { Silhouette } \\
\text { value }\end{array}$ \\
\hline $\begin{array}{l}\text { Proposed } \\
\text { algorithm } \\
\text { based } \\
\text { islanding }\end{array}$ & 1 & 33.41 & 328.2 & 137.7 & 0.781 \\
\hline $\begin{array}{l}\text { Commu- } \\
\text { nity } \\
\text { detection } \\
\text { based } \\
\text { islanding }\end{array}$ & 3 & 120.94 & 339.03 & 147.03 & 0.3061 \\
\hline
\end{tabular}

trajectories are of different length; however, since correlation method is a point-to-point distance-based method, it requires an equal length of trajectories to compute the correlation matrix for coherency evaluation. Further, practical application of community detection method is also challenging for online identification of coherent groups with partial observability of the system [116].

\subsubsection{Western interconnection power system of North America}

A Modified version of Western Interconnection power system of North America is also employed to validate and demonstrate the performance of proposed coherency determination method. It has 29 synchronous generators. System's topological structure and parameters can be found in [55]. Rotor angle trajectories' data of nine poorly damped oscillation cases, named as $N D_{1}, N D_{2}, \ldots, N D_{9}$ are utilized to signify the performance and comparison of the proposed coherency identification with HC [87], and ICA [81] methods. Coherency identification results of the proposed, ICA and HC methods along with their average silhouette values are shown in Table 8. Generators' sets are formed as $A=\{103,112,116,118\}, B=\{13,15,40,43,47,138,140,144,148,149\}, C=$ $\{30,35,65,70,77,79\}, D=\{4,6,9,11,18,36,45,159,162\}, D 1=\{4,6,9,11,18\}, D 2=$ $\{36,45,159,162\}$ [111].

Higher value of the metric average silhouette value $\left(V_{i s}\right)$ indicates the better generators' coherency identification. As can be seen from Table 3.9, the proposed coherency method has a higher average silhouette value of each case than HC and ICA methods. It signifies that the generators identified by the proposed method are more strongly matched to its coherent group and poorly matched to its neighboring groups. Hence, the proposed coherency method is effective to determine generators' coherency considering the average silhouette value. 
Table 3.9 Performance comparison between proposal and community detection based algorithms

\begin{tabular}{|c|c|c|c|}
\hline Cases & Proposed Approach & HC[48] & ICA [10] \\
\hline ND_1 & $\begin{array}{l}\mathrm{V}_{i}^{s}=0.8579, C G 1= \\
D, C G 2=C \bar{G} 1\end{array}$ & $\begin{array}{l}\mathrm{V}_{i}^{s}=0.5559, C G 1= \\
\{45,159\}, C G 2= \\
C \bar{G} 1\end{array}$ & $\begin{array}{l}\mathrm{V}_{i}^{s}=0.8502, C G 1= \\
C G 2+C G 3, C G 2= \\
\{45,159\}, C G 3= \\
\{D-C G 2\}\end{array}$ \\
\hline ND_2 & $\begin{array}{l}\mathrm{V}_{i}^{s}=0.6519, C G 1= \\
\{36,116,118\}, C G 2= \\
\{30,35,65,79\}, C G 3= \\
B+D 1, C G 4= \\
\{45,103,159,162\}, C Q \\
\{70,77,112\}\end{array}$ & $\begin{array}{l}\mathrm{V}_{i}^{s}= \\
-0.1717, C G 1= \\
5\{\underline{=}=, C G 2=C \bar{G} 1\end{array}$ & $\begin{array}{l}\mathrm{V}_{i}^{s}=0.5782, C G 1= \\
A+D 2, C G 2= \\
B+D 1, C G 3=C\end{array}$ \\
\hline ND_3 & $\begin{array}{l}\mathrm{V}_{i}^{s}=0.8024, C G 1= \\
C, C G 2=C \bar{G} 1\end{array}$ & $\begin{array}{l}\mathrm{V}_{i}^{s}= \\
-0.2438, C G 1= \\
\{6,11\}, C G 2=C \bar{G} 1\end{array}$ & $\begin{array}{l}\mathrm{V}_{i}^{s}=0.5273, C G 1= \\
C G 2+C G 3, C G 2= \\
B+D 1, C G 3=\{65\}\end{array}$ \\
\hline ND_4 & $\begin{array}{l}\mathrm{V}_{i}^{s}=0.7553, C G 1= \\
\{13,15,138,148\}, C G 2 \\
D, C G 3=C, C G 4= \\
B-G 1\end{array}$ & $\begin{array}{l}\underline{\mathrm{V}}_{i}^{s}= \\
=0.3386, C G 1= \\
\{6,11\}, C G 2=C \bar{G} 1\end{array}$ & $\begin{array}{l}\mathrm{V}_{i}^{s}=0.6221, C G 1= \\
C G 2+C G 3, C G 2= \\
D-\{6,11\}, C G 3= \\
\{6,11\}\end{array}$ \\
\hline ND_5 & $\begin{array}{l}\mathrm{V}_{i}^{s}=0.7826, C G 1= \\
C+\{112\}, C G 2= \\
C \bar{G} 1\end{array}$ & $\begin{array}{l}\mathrm{V}_{i}^{s}= \\
-0.1541, C G 1= \\
\{6,11\}, C G 2=C \bar{G} 1\end{array}$ & $\begin{array}{l}\mathrm{V}_{i}^{s}=0.5273, C G 1= \\
C G 2+C G 3, C G 2= \\
B+D 1, C G 3=\{65\}\end{array}$ \\
\hline ND_6 & $\begin{array}{l}\mathrm{V}_{i}^{s}=0.8706, C G 1= \\
D, C G 2=C \bar{G} 1\end{array}$ & $\begin{array}{l}\mathrm{V}_{i}^{s}= \\
-0.5735, C G 1= \\
\{45,159\}, C G 2= \\
C \bar{G} 1\end{array}$ & $\begin{array}{l}\mathrm{V}_{i}^{s}=0.8535, C G 1= \\
C G 2+C G 3, C G 2= \\
D-\{6,11\}, C G 3= \\
\{6,11\}\end{array}$ \\
\hline ND_7 & $\begin{array}{l}\mathrm{V}_{i}^{s}=0.8578, C G 1= \\
D, C G 2=C \bar{G} 1\end{array}$ & $\begin{array}{l}\mathrm{V}_{i}^{s}= \\
-0.5180, C G 1= \\
\{45,159\}, C G 2= \\
C \bar{G} 1\end{array}$ & $\begin{array}{l}\mathrm{V}_{i}^{s}=0.8439, C G 1= \\
C G 2+C G 3, C G 2= \\
D-\{6,11\}, C G 3= \\
\{6,11\}\end{array}$ \\
\hline ND_8 & $\begin{array}{l}\mathrm{V}_{i}^{s}=0.8533, C G 1= \\
D, C G 2=C \bar{G} 1\end{array}$ & $\begin{array}{l}\mathrm{V}_{i}^{s}= \\
-0.5401, C G 1= \\
\{45,159\}, C G 2= \\
C \bar{G} 1\end{array}$ & $\begin{array}{l}\mathrm{V}_{i}^{s}=0.8545, C G 1= \\
C G 2+C G 3, C G 2= \\
D-\{6,11\}, C G 3= \\
\{6,11\}\end{array}$ \\
\hline ND_9 & $\begin{array}{l}\mathrm{V}_{i}^{s}=0.8698, C G 1= \\
\{70,77,112\}, C G 2= \\
B+D 1+\{103\}- \\
\{6,11\}, C G 3= \\
\{35,65\}, C G 4= \\
\{6,11,45,159,162\}, C \\
\{36,116,118\}, C G 6= \\
\{30,79\}\end{array}$ & $\begin{array}{l}\mathrm{V}_{i}^{s}=0.1651, C G 1= \\
\{6,11\}, C G 2=C \bar{G} 1 \\
G 5=\end{array}$ & $\begin{array}{l}\mathrm{V}_{i}^{s}=0.8575, C G 1= \\
C \bar{G} 2, C G 2=B+D 1\end{array}$ \\
\hline
\end{tabular}


The proposed DTW based coherency identification approach can be integrated into the wide area monitoring system in the control center. Coherent generator groups can be determined from PMU measurements and provide wide-area monitoring and control for controlled islanding.

\subsection{Performance of proposed approach with partial observability and noise}

The performance of online PMU measurements-based algorithms is sensitive to partial loss or delay. In PMU based wide area measurement system, communication link failure is common, which may lead the system to be partially observable. Monitoring and control with incomplete information may result in misoperation. Hence, it is important to ensure that the coherency identification method is robust to some extent against partial loss/delay of PMU data. Moreover, due to the ever-decreasing cost of PMUs, as compared to benefits gained in the form of increased system observability, their deployment is massively increasing. This increased dependency on PMUs also poses some challenges for online approaches in case of partial observability of the system. This area has not been widely explored, specifically for online coherency identification application. Some researchers also reported it as the limitation of their proposed coherency identification approach [71].

The proposed online coherency approach is applicable in the case of partial observability of the system due to its non-linear nature of similarity computation as explained in Section 3.2. Consider case 1 mentioned in Section 3.3, where we have cascaded outages of line 17-16 and 2-1 at $\mathrm{t}=5 \mathrm{~s}$ and $\mathrm{t}=7 \mathrm{~s}$ respectively. We considered PMUs on generator buses only. To analyze the performance of proposed online coherency algorithm for a partially observable system, we intentionally remove the initial measurement points for each PMU. Figure 3.14 shows the experimental results. The green color in each curve indicates the lost part of PMU data. We determine the coherency through proposed approach. After determining the coherency with such incomplete PMU data, we compare the coherency results with the results obtained without loss of measurements. The algorithm still gives us the same coherency results. Thus, the proposed online coherency approach is robust to a considerable extent for loss/delay of PMU data.

Here, we considered the worst-case scenario when a certain consecutive portion of PMU measurements is lost. Measurement loss randomly is an easier problem to solve as compared to the one discussed in this chapter. Further, we considered measurement loss rate ranging 5\% - 45\% for different PMU channels as shown in Fig. 3.14.

To validate the applicability of the proposed coherency identification method for actual PMU measurements in the presence of noise, white Gaussian noise is added for simulating the measurement noise. 


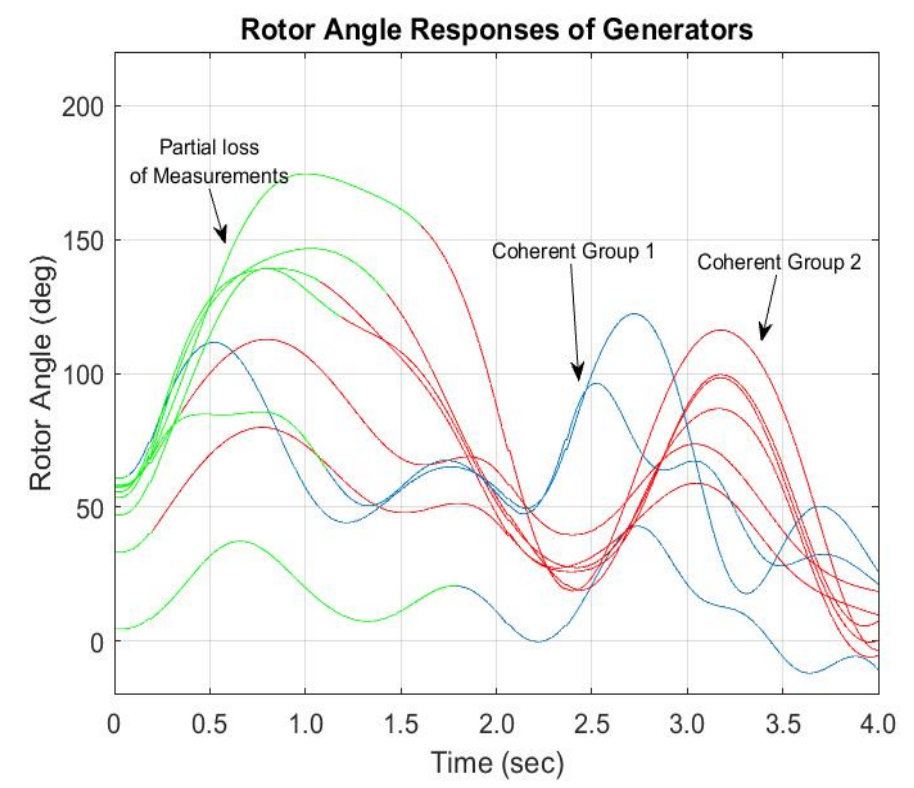

Fig. 3.14 Performance of proposed coherency algorithm with partial loss of PMU data

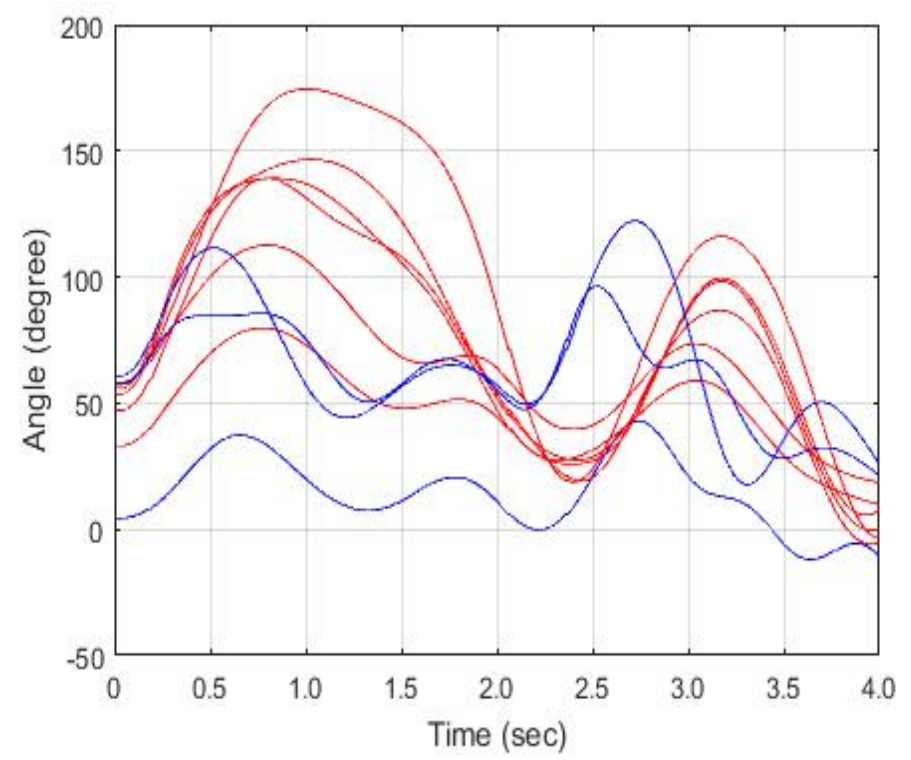

Fig. 3.15 Generators' coherency grouping identified by proposed coherency method without noise

Rotor angle trajectories and their coherency grouping as suggested by the proposed coherency method are shown in Fig. 3.15. Reference [117] experimentally recommended an SNR value of $45 \mathrm{~dB}$ as a good approximation for real PMU data. However, we test the performance with a higher level of noise, i.e., $30 \mathrm{~dB}$. Figure 3.16 shows the generator grouping identified by the proposed coherency method in the presence of noise. It can be seen that the coherency grouping with white Gaussian noise is the same as the one without noise. Hence, the performance of the proposed method is not affected by a certain level of noise. 


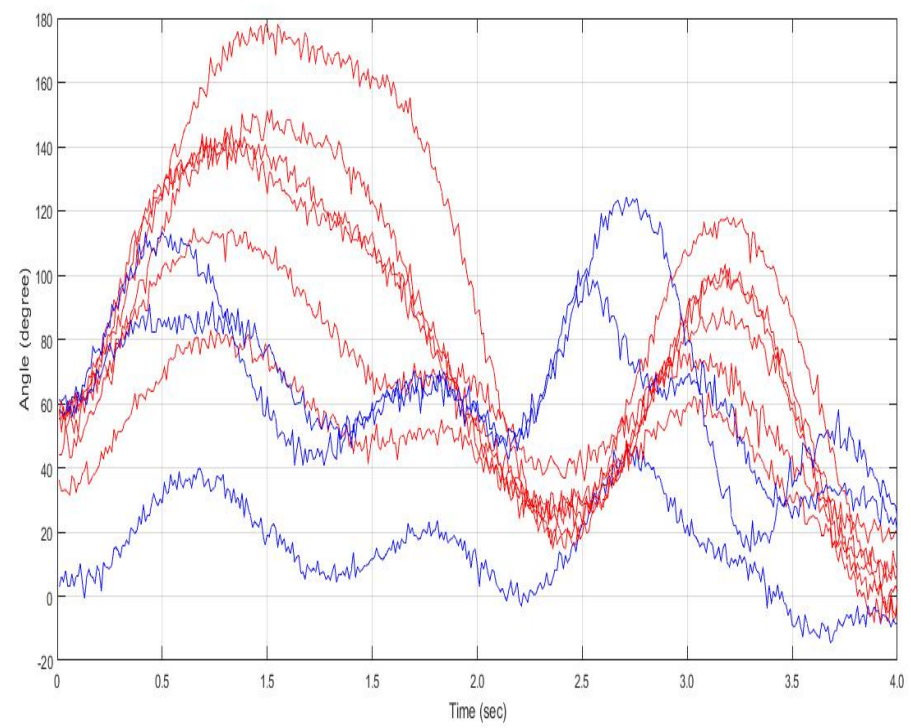

Fig. 3.16 Generators' coherency grouping identified by proposed coherency method in the presence of white Gaussian noise

\subsection{Conclusion}

Splitting the system into self-sustained islands is the last resort to maintain transient stability. This chapter presents a novel methodology for generator coherency identification. It uses post-fault rotor angle trajectories of generators for coherency determination. For non-generator buses allocation, constrained spectral clustering is applied to minimize power flow disruption, considering coherency matrix as a constraint. Future work includes: allocation of non-generator buses based on multiple constraints like restoration constraint, thermal limits of transmission lines, etc., in addition to generator coherence constraint; prevention of blackouts using energy storage system without going into islanding operation mode; further testing of the proposed methodology on real-time simulator for hardware-in-the-loop simulation. 


\section{CHAPTER 4}

\section{Coherence Identification using Structural Characteristic Measures of PMU Time Series Data}

\subsection{Introduction}

The use of phasor measurement unit in complex interconnected power system for monitoring and control has increased significantly. This has resulted in huge amounts of data and growing databases. Moreover, the expansion of power grid due to regional interconnections and the increase in diversity of the transmission structure owing to ever increasing market competition have made safety and stability of the system very crucial and challenging. The dynamic stability and security assessment of such large and complex interconnected power systems becomes very challenging with detailed system modeling and requires new technologies which rely on the online data obtained through wide area measurement and control system [118, 119]. Moreover, with increasing energy demand, more energy resources are continuously being added in the existing network [120, 121, 122, 123]. This complex network thus operates comparatively closer to its stability limits with minimal flexibility and reliability. Such conditions may lead to small signal instability causing power fluctuations and discontinuity of supply in some cases. Not only disturbances caused by natural calamities such as hurricanes and earthquakes but also operational mistakes may trigger cascading failures, which may result in system wide blackouts and pose a significant threat to properties and lives. When a power system encounters a disturbance or a small change in operating state, under stressed condition, it experiences electromechanical oscillations. These oscillations may exist between machines operating in a plant (local area) or between the plants in distant areas, in the form of inter-area oscillations [5]. During inter area oscillation, system generators tend to operate in groups oscillating against each other. The generators operating in one group are called coherent generators. The identification of such coherent groups is critical to ensure dynamic security and designing appropriate countermeasures for maintaining system stability during controlled islanding [124, 125]. In

addition, coherency determination is also crucial for transient stability studies of large interconnected power system via developing dynamic equivalents [126]. The significance of fast and accurate identification of generators' coherent groups using wide-area 
measurements lies in its contribution to the design of WAMC based security and stability control schemes that intent to enhance the overall performance of power system.

This chapter proposes an approach for data driven coherency determination using rotor angle trajectories' characteristic measures. On the contrary, when the coherency identification algorithm is based on a distance metric [127, 128, 129, 130, 131], it cannot handle time series with missing data or of different lengths since actual points on the trajectories are used as inputs for similarity evaluation. However, by extracting a set of characteristic measures from the original time series trajectories this problem is simply bypassed. Moreover, characteristic measures extraction process can also be considered as a dimensionality reduction procedure in time series data mining [132]. The effectiveness of the proposed structural cumulative characteristic measure (CCM) based coherence identification approach has been validated and compared with hierarchical clustering (HC) method [128] for Western interconnection power system in North America (WECC) 179-bus system.

\subsection{Characteristic Measures of Trajectories for Coherency Identification}

The use of phasor measurement units in complex interconnected power system for monitoring and control has increased significantly. Given voltage and current phasor measurements at generator terminal buses, rotor angle trajectories of these generators $\delta$ can be estimated using least squares (LS) or Kalman filter (KF) based approaches as shown in Fig. 4.1. Consider rotor angle trajectories $\delta_{m n}=\left\{\delta_{1 n}, \delta_{2 n}, \ldots, \delta_{m n}, \ldots, \delta_{M n}\right\}, \forall m \in$ $\{1,2,3, \ldots, M\}$ and $\forall n \in\{1,2,3, \ldots, N\}$, where $\delta_{m n}$ is the $n^{\text {th }}$ sampling time of $m^{\text {th }}$ generator trajectory [133]. It is to be noted that two generators are considered to be coherent if their time domain rotor angle response trajectories are similar. Here, characteristic measures of rotor angle trajectories, are utilized to determine the similarity among trajectories for coherency identification as described below.

\subsubsection{Skewness}

Skewness is a measure of symmetry, or more precisely, the lack of symmetry. A distribution, or dataset, is symmetric if it looks the same to the left and to the right of the center point. It is used to characterize the degree of asymmetry of values around the mean value. Mathematically, skewness Sk is defined as the standardized third moment of a distribution and described as:

$$
S k=E\left[\left(\frac{\delta_{m n}-\mu}{\sigma}\right)^{3}\right]=\frac{E\left[\left(\delta_{m n}-\mu\right)^{3}\right]}{\left(E\left[\left(\delta_{m n}-\mu\right)^{2}\right]\right)^{\frac{3}{2}}}=\frac{\mu^{3}}{\sigma^{2}}
$$

where $\mu$ is the mean, $\sigma$ is the standard deviation, $\mathrm{E}$ is the expectation operator and $\sigma^{3}$ is the third central moment. For a generator $m$ with rotor angle trajectory $\delta_{m n}$ having $n$ 


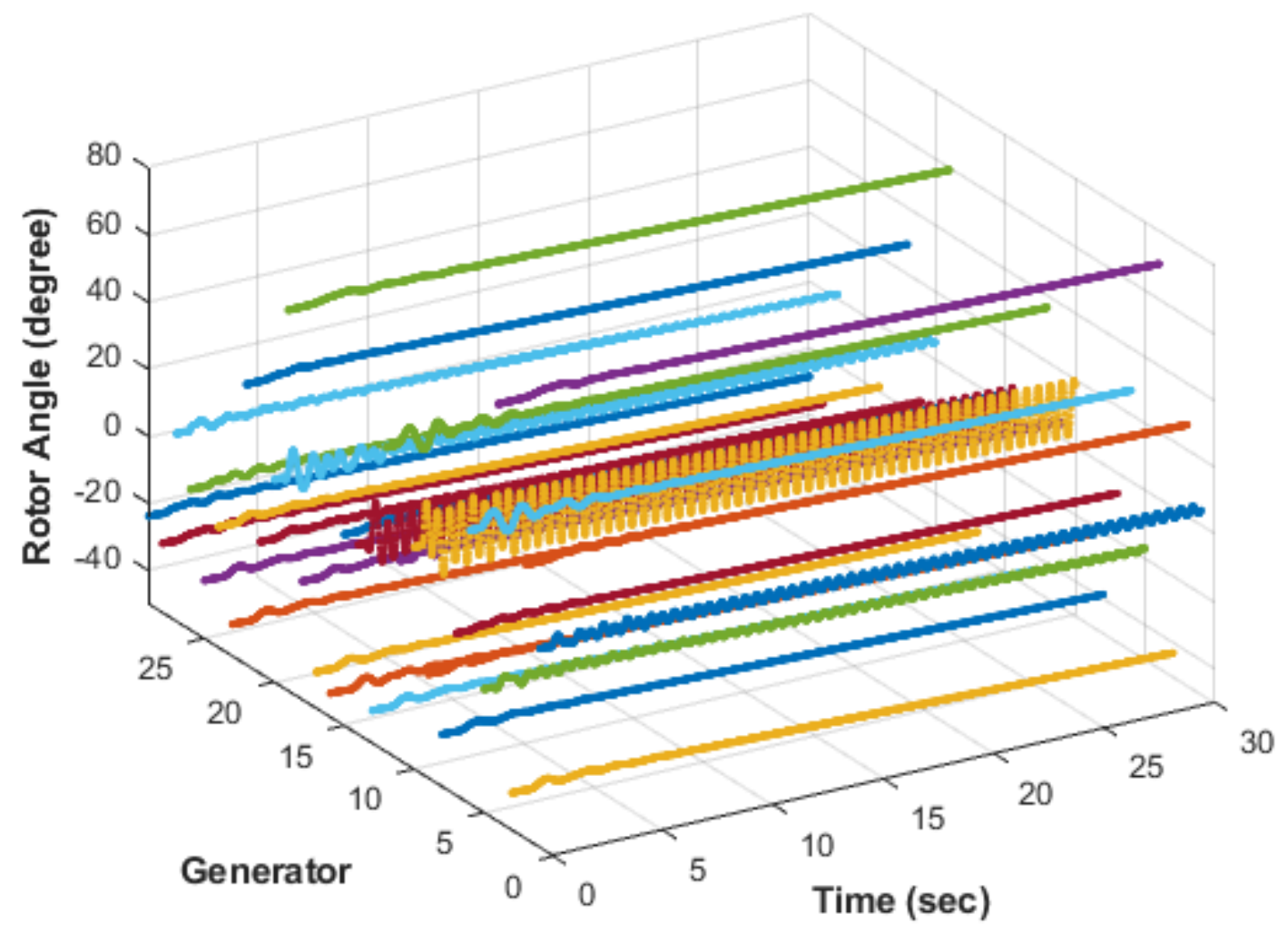

Fig. 4.1 Rotor angle trajectories of generators in WECC 179 system following a disturbance at $0.5 \mathrm{sec}$

samples, the skewness Sk can be computed as [134]:

$$
S k=\frac{\frac{1}{N} \sum_{n=1}^{N}\left(\delta_{m n}-\overline{\delta_{m}}\right)^{3}}{\sqrt[3]{\frac{1}{N} \sum_{n=1}^{N}\left(\delta_{m n}-\overline{\delta_{m}}\right)^{2}}}=\frac{\frac{1}{N} \sum_{n=1}^{N}\left(\delta_{m n}-\overline{\delta_{m}}\right)^{3}}{\left[\frac{1}{N} \sum_{n=1}^{N}\left(\delta_{m n}-\overline{\delta_{m}}\right)^{2}\right]^{\frac{3}{2}}}
$$

The skewness for a normal distribution is zero, and any symmetric time series data should have the skewness near zero. But in reality, PMU data samples may not be perfectly symmetric. So, the skewness of a PMU reported time series dataset indicates whether deviations from the mean are going to be positive or negative. Negative values for the skewness indicate the PMU data that is skewed left, and positive values for the skewness indicate the PMU data that is skewed right.

\subsubsection{Self-Similarity}

The definition of self-similarity in terms of time series data is expressed as a selfsimilarity parameter called Hurst exponent $(\mathrm{H})$. Hurst exponent is a measure to express long-term memory of a time series. It is related to autocorrelations of the time series, and the rate at which these decrease as the lag between pairs of values increases. The 
Hurst exponent is defined in terms of the behavior of the rescaled range as a function of the time span of a time series as follows [135]:

$$
E\left[\frac{R(n)}{\sigma(n)}\right]=C n^{H}
$$

where $R(n)$ is the range of the first $n$ cumulative deviations from the mean, and $S(n)$ is their standard deviation. $E(n)$ is the expectation operator, $n$ is the number of data points in the time series and $C$ is a constant.

Hurst exponent is also referred as the "measure of dependence" or "measure of longrange dependence". It quantifies the relative tendency of a PMU reported time series data either to regress strongly to the mean or to cluster in a direction [136]. A value in the range $[0.5,1]$ indicates a PMU time series with long-term positive autocorrelation, meaning a high value in the series will be followed by another high value. A value in the range $[0,0.5]$ indicates a PMU time series with long-term switching between high and low values in adjacent pairs, meaning that a single high value will be followed by a low value. A PMU measured time series having a value of $\mathrm{H}=0.5$ indicates a completely uncorrelated series.

\subsubsection{Kurtosis}

Kurtosis is a measure of the "tailedness" of a distribution of a real-valued time series. It is based on the fourth moment of the data and is a descriptor of the shape of the probability distribution, and can be regarded as a measure of deviation from Gaussian distribution. Mathematically, kurtosis $K$ is defined as the standardized fourth moment around the mean of a distribution:

$$
K=E\left[\left(\frac{\delta_{m n}-\mu}{\sigma}\right)^{4}\right]=\frac{E\left[\left(\delta_{m n}-\mu\right)^{4}\right]}{\left(E\left[\left(\delta_{m n}-\mu\right)^{2}\right]\right)^{2}}=\frac{\mu^{4}}{\sigma^{4}}
$$

where $E$ is the expectation operator, $\mu$ is the mean, $\mu^{4}$ is the fourth moment and $\sigma$ is the standard deviation. The kurtosis $K$ of a generator $m$ with rotor angle trajectory $\delta_{m n}$ having $n$ samples can be computed as [137]:

$$
K=\frac{\frac{1}{N} \sum_{n=1}^{N}\left(\delta_{m n}-\overline{\delta_{m}}\right)^{4}}{\left(\frac{1}{N} \sum_{n=1}^{N}\left(\delta_{m n}-\overline{\delta_{m}}\right)^{2}\right)^{2}}
$$

For symmetric distributions, positive kurtosis indicates heavy tails and peakedness relative to the normal distribution, while negative kurtosis indicates light tails and flatness. Kurtosis is a measure of whether the PMU reported time series data is peaked or flat, relative to a normal distribution. A PMU measured time series with high kurtosis tends to have a distinct peak near the mean, decline rather rapidly, and have heavy tails. On the contrary, a PMU time series dataset with low kurtosis tends to have a flat top near the mean rather than a sharp peak. 


\subsubsection{Approximate Entropy}

Approximate entropy $(A p E n)$ is a characteristic measure to quantify the amount of regularity of fluctuations over time series data. It is a computationally simple and efficient estimate of entropy that measures the so-called "pattern similarity" in the time series. Regularity is originally measured by regularity measures like entropy but accurate entropy calculation requires vast amounts of data, therefore it is not practical in the coherency problem since the coherency might change quickly during cascading failures. To compute the ApEn for $m_{t h}$ rotor angle trajectory $\delta_{m n}$ with $N$ samples, similarity between two sub-patterns $\delta_{m n}(i)$ and $\delta_{m n}(j)$ is found as $\left|\delta_{m n}(i+k)-\delta_{m n}(j+k)<r\right|$ for $0 \leq k<s$; where $s$ and $r$ specify the pattern length and criteria of similarity respectively [138]. In addition, $i$ and $j$ are the beginning indexes of sub-patterns $\delta_{m n}(i)$ and $\delta_{m n}(j)$ respectively. Now consider a pattern $\delta_{m n}(n-s+1)$ within $\delta_{m N}$ for which we can define:

$$
C_{i s}(r)=\frac{n_{i s}(r)}{N-s+1}
$$

where $n_{i s}(r)$ is the number of patterns in $\delta_{m N}$ that are similar to $\delta_{m n}(i) . C_{i s}(r)$ is the fraction of patterns of length $s$ that resemble all patterns beginning from instant $i$ and have the same length. We can compute $C_{i s}(r)$ for each sub-pattern in $\delta_{m N}$ and define a mean of these as $C_{s}(r)$. This mean value indicates the prevalence of repetitive subpatterns of length $s$ in trajectory $\delta_{m N}$. Finally, the approximate entropy of $\delta_{m N}$ for sub-pattern of length $s$ with similarity criteria $r$ can be defined as:

$$
A p \operatorname{En}\left(\delta_{m N}, s, r\right)=\ln \frac{C_{s}(r)}{C_{s+1}(r)}
$$

A PMU measured time series trajectory containing many repetitive sub-patterns has a relatively small ApEn and a less predictable dynamic PMU time series has a higher ApEn.

\subsection{Proposed Cumulative Characteristic Measure based Coherency Iden- tification}

From the characteristic measures presented in section 4.2, four $N \times M$ matrices $\mathbf{F}_{\mathbf{c}}$ $=\left[F_{c}(n, m)\right]_{N x M}$ can be constructed with $M$ rotor angle trajectories having $N$ samples, where $c$ is the number of characteristic measures. These measures can not be compared with each other due to their different intrinsic nature. Therefore, the normalization of these measures needs to be done first. The normalization in the range $[0,1]$ can be formulated as:

$$
F_{c}^{*}(n, m)=\frac{F_{c}^{*}(n, m)-\min _{m, n}\left\{F_{c}^{*}(n, m)\right\}}{\max _{m, n}\left\{F_{c}^{*}(n, m)\right\}-\min _{m, n}\left\{F_{c}^{*}(n, m)\right\}}
$$


where $F_{c}^{*}(n, m)$ is the normalized $c^{t h}$ characteristic measure. The normalized characteristic measures $F_{c}^{*}(n, m), \forall c \in\{1,2,3,4\}$ can be integrated into a Cumulative Characteristic Measure (CCM) matrix as:

$$
F_{C C M}^{*}(n, m)=\sum_{c=1}^{4} w_{c} F_{c}^{*}(n, m)
$$

where $w_{c}$ is the weight of the $c_{t h}$ characteristic measure to form cumulative characteristic measure. In this chapter, equal weights are assumed for all characteristic measures such that $\sum_{c=1}^{4} w_{k}=1$. This cumulative measure is used to identify the similarity of the rotor angle trajectories. The advantage of forming such characteristic based cumulative measure is it's tolerance for missing data.

Coherent generator groups can be identified by finding the similarity between their rotor angle response trajectories. Similarity is expressed in terms of correlation between cumulative characteristic measure of generators. Pearson correlation coefficient $\rho$ is utilized to compute this correlation. It describes the linearity between two generators cumulative characteristic measures $F_{C C M}^{*}(n, p), F_{C C M}^{*}(n, q)$ and expressed as:

$$
\rho_{p, q}=\frac{\operatorname{cov}\left(F_{C C M}^{*}(n, p), F_{C C M}^{*}(n, q)\right)}{\sigma_{F_{C C M}^{*}(n, p)} \sigma_{F_{C C M}^{*}(n, q)}}
$$

where $\operatorname{cov}\left(F_{C C M}^{*}(n, p), F_{C C M}^{*}(n, q)\right)$ is the covariance between cumulative characteristic measures $F_{C C M}^{*}(n, p)$ and $F_{C C M}^{*}(n, q)$. And $\sigma_{F_{C C M}^{*}(n, p)}$ and $\sigma_{F_{C C M}^{*}(n, q)}$ are the standard deviations of cumulative characteristic measures for generators $p$ and $q$ respectively. This results in an $M \times M$ similarity matrix $\mathbf{S}$.

$$
S=\left[\begin{array}{cccc}
\rho_{1,1} & \rho_{1,2} & \cdots & \rho_{1, M} \\
\rho_{2,1} & \rho_{2,2} & \cdots & \rho_{2, M} \\
\vdots & \vdots & \ddots & \vdots \\
\rho_{M, 1} & \rho_{M, 2} & \cdots & \rho_{M, M}
\end{array}\right]
$$

The next step is to cluster coherent generators using cumulative characteristic measure based similarity matrix $\mathbf{S}$ constructed in (4.11). Using $\mathbf{S}$, the optimal number of coherent groups $k$ is obtained by minimizing inter-coherent group distances. The proposed cumulative characteristic measure (CCM) based coherency identification scheme, can be implemented using the following steps.

Step 1: Estimation of generators' rotor angles based on PMU measurements of voltage and current at each generator terminal bus.

Step 2: Characteristic measures extraction from rotor angle trajectories using (4.2), (4.3), (4.5) and (4.7).

Step 3: Normalization of the characteristic measures obtained in step 2 using (4.8) for better comparability. 


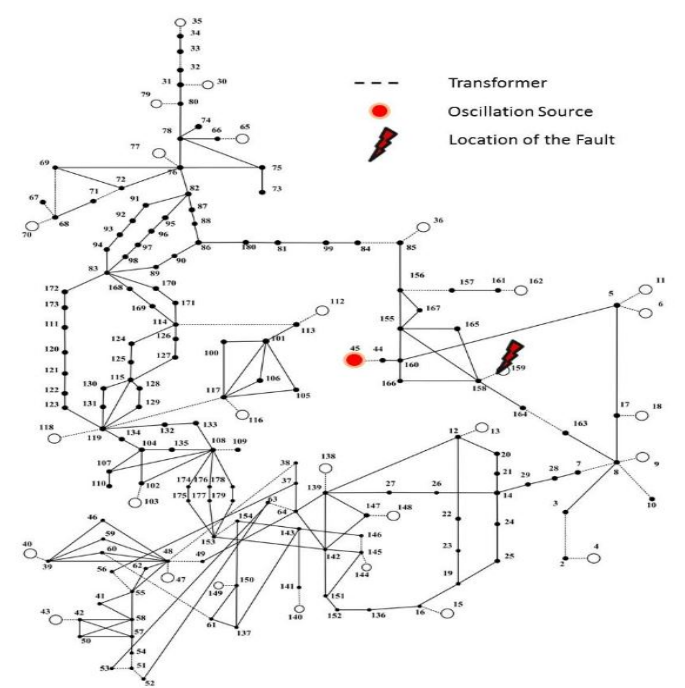

Fig. 4.2 Three phase short circuit fault at bus 159 in WECC 179-bus system

Step 4: Evaluation of weights of the characteristic measures to form cumulative characteristic measure (CCM) using (4.9).

Step 5: Similarity evaluation between rotor angle trajectories using cumulative characteristic measure via Pearson correlation coefficient presented in (4.10). It defines a matrix of similarity index for each pair of generators as in (4.11) .

Step 6: Finding the optimal number of coherent groups $(k)$ by minimizing inter-coherent group distances. It provides the number of unique coherent groups.

Step 7: Grouping of generators using k-means into $k$ coherent groups.

\subsection{Experimental Validation and Performance Evaluation}

A modified version of Western Interconnection power system of North America is employed to validate and demonstrate the performance of proposed coherency identification method. It has 29 synchronous generators. System's topological structure and parameters can be found in [43].

Silhouette measure can be used to validate the coherent group formation identified by coherency determination algorithms, which is a measure to validate the consistency of the clusters in the data [139] -[140]. Utilizing this index, it can be shown that how strongly generators in one group are coherent and coupled together as compared to those in other coherent groups. It is expressed as:

$$
V_{i}^{S} \cong \begin{cases}\frac{D_{\text {min,avg }}(i)-D_{\text {avg }}(i)}{D_{\text {min }, a v g}(i)} & D_{\text {avg }}(i)<D_{\text {min,avg }}(i) \\ 0 & D_{\text {avg }}(i)=D_{\text {min,avg }}(i) \\ \frac{D_{\text {min,avg }}(i)-D_{\text {avg }}(i)}{D_{\text {avg }}(i)} & D_{\text {avg }}(i)>D_{\text {min,avg }}(i)\end{cases}
$$

where $D_{\text {min,avg }}(i)$ is the minimum average dissimilarity of $i_{t h}$ generator with respect 


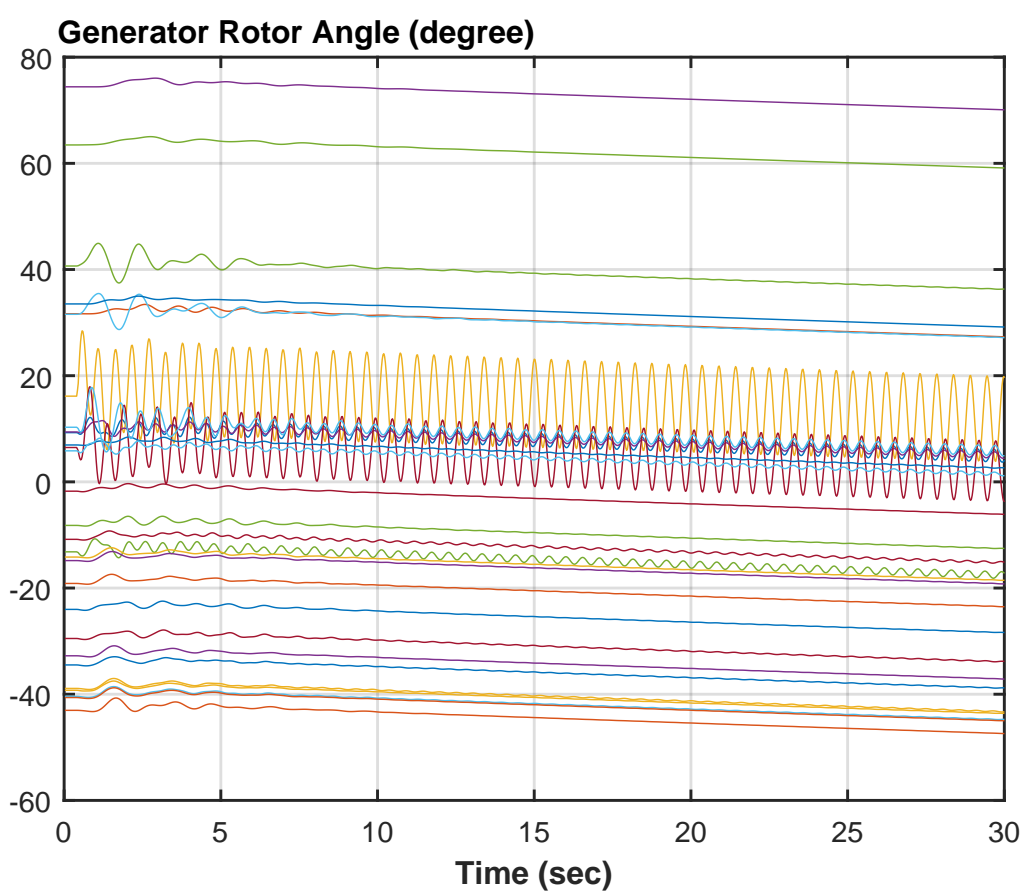

Fig. 4.3 Rotor angle trajectories of 29 generators in WECC 179 system following a three phase short circuit fault

to other coherent groups, and $D_{\text {avg }}(i)$ is the average dissimilarity of $i_{t h}$ generator with respect to all other generators in the same coherent group. A generator with large silhouette value $\left(V^{S}\right)$ indicates that it is strongly coupled with the generators of its coherent group and weakly coupled with the neighboring groups. Generators coherency identification is appropriate if most of the generators have large silhouette values. The criteria to determine the most suitable coherent generators' group formation is to check the average silhouette value [141].

The coherent groups' formation having higher average silhouette value $\left(V_{a}^{S}\right)$ will indicate more strongly coupled coherent groups [142]. More strongly coupled coherent generator groups will in turn ensure more transient stability after islanding and will help in healing the system and avoiding a blackout [142]. A few researchers have recently used this coherency validation criterion to evaluate coherent generators groups formation [141] - [142]. Therefore, it can be regarded as a measure of transient stability of the newly formed islands during islanding.

Table 4.1 Allocation of Generators into Coherent Groups

\begin{tabular}{cc}
\hline \hline Coherent Group 1 (CG1) & Coherent Group 2 (CG2) \\
\hline $1,2,4,5,7,9,10,11,13,14,16,17$ & $3,6,8,12,15$, \\
$18,19,20,21,22,23,24,25,27,29$ & 26,28 \\
\hline
\end{tabular}




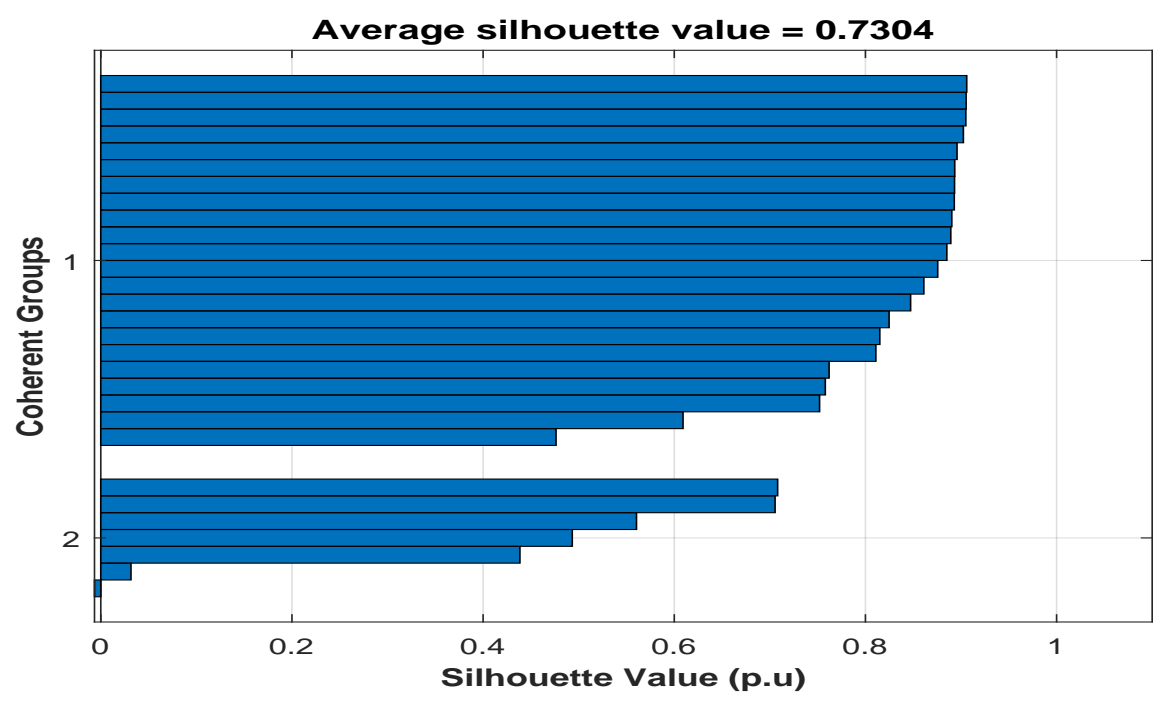

Fig. 4.4 Silhouette plots for coherent generator groups

\subsubsection{Experimental Validation}

A three-phase short circuit fault is applied at bus 159 at $0.5 \mathrm{~s}$ and cleared after $0.05 \mathrm{~s}$ as shown in Fig.4.2. Resulting rotor angle trajectories are shown in Fig.4.3. The proposed CCM based coherency identification approach suggested two coherent groups listed in Table 4.1. As mentioned above, average silhouette measure is used to validate the closeness of coherent generator groups and in turn quantifies the stability of coherent groups. Average silhouette measure for this case study is $V_{a}^{S}=0.7304$ as shown in Fig.4.4. This coherency quality measure is computed considering all four characteristic measures presented in section 4.2. We also analyzed the change in the coherency quality measure with the number of characteristic measures considered for CCM construction as shown Fig.4.5. It indicates that considering approximate entropy alone results coherency quality measure in the form of average silhouette value of $V_{a}^{S}=0.4069$ and along with kurtosis, rises up to $V_{a}^{S}=0.6738$. Adding skewness further it rises to $V_{a}^{S}=0.7024$ and eventually considering all four characteristic measures, it reaches to $V_{a}^{S}=0.7304$ and indicates strongly coupled coherent generator groups formation.

\subsubsection{Performance Comparison}

Rotor angle trajectories' data of nine poorly damped oscillation cases, named as $N D \_1$, $N D \_2, \ldots, N D \_9$, are utilized to signify the performance and comparison of the proposed coherency identification with $\mathrm{HC}$ method. Coherency quality measure is compared, in the form of average silhouette value, for the proposed and the existing HC method [128] and given in Table 4.2. Higher value indicates better generators' coherency identification. As can be seen from Table 4.2, the proposed CCM method has a higher coherency quality measure value in each case as compare to HC method. It signifies that the generators identified by the proposed method are more strongly matched to 


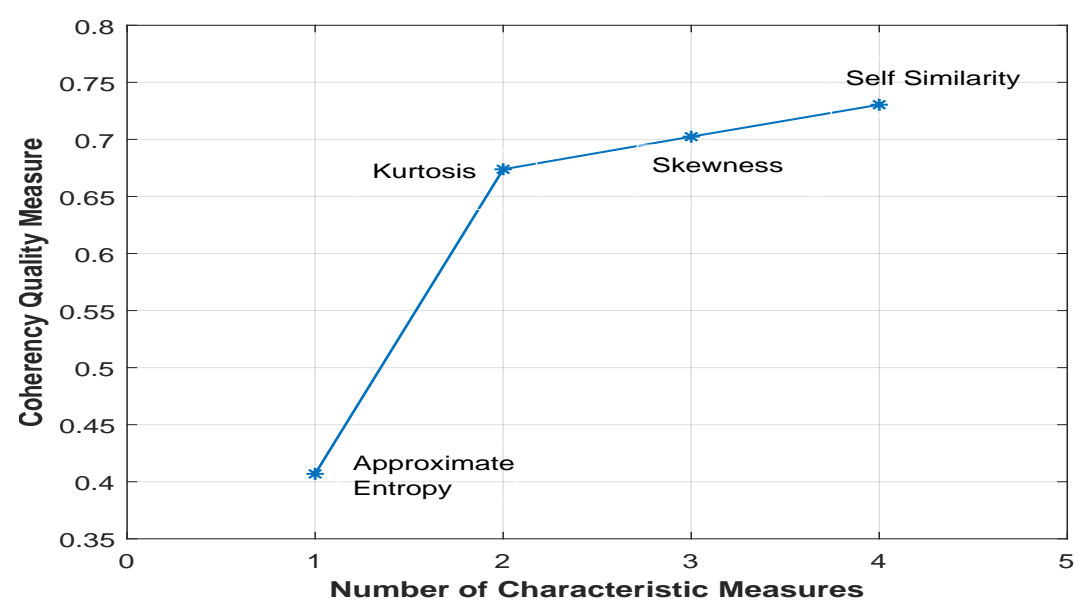

Fig. 4.5 Coherency quality measure changes as characteristic measures are added Table 4.2 Comparison of proposed characteristic measures based coherency method for Western interconnection power system of North America

\begin{tabular}{ccc}
\hline \hline & \multicolumn{2}{c}{ Coherency Quality Measure } \\
Case & Proposed CCM Method & HC Method [128] \\
\hline ND_1 & 0.7304 & 0.5559 \\
ND_2 & 0.6747 & -0.1717 \\
ND_3 & 0.5281 & -0.2438 \\
ND_4 & 0.4243 & 0.3386 \\
ND_5 & 0.7014 & -0.1541 \\
ND_6 & 0.7516 & 0.5735 \\
ND_7 & 0.6490 & 0.5180 \\
ND_8 & 0.6771 & 0.5401 \\
ND_9 & 0.4308 & 0.1651 \\
\hline
\end{tabular}

their coherent groups and poorly matched to neighboring groups. Hence, the proposed CCM based method is effective to determine generators' coherency.

\subsection{Conclusion}

This chapter presents a novel methodology for generators coherency identification. It uses structural characteristic measures of rotor angle trajectories for coherency determination. The proposed coherency identification approach can be integrated into the wide area monitoring system in the control center to provide system operator a tool to evaluate generators' coherency for controlled islanding. 


\section{CHAPTER 5}

\section{Recommendations for Future Work}

The following suggestions are made for any continuing research based on the results presented in this study.

- Power electronic based inverters are used to feed in the electrical energy from renewable sources to the electrical grid in a microgrid. Unlike synchronous machine, inverters don't possess any mechanical torque due to absence of rotating mass, and hence can not provide inertia to the system. Compared to gridconnected mode, a microgrid in islanded mode may experience various stability issues. Further, lack of spinning reserves and low physical inertia make its dynamic response quicker than conventional rotating machines. This increases islanded microgrid's susceptibility towards oscillations resulting from highly fluctuating loads, intermittent renewable sources or malfunctioning of feedback controllers [143]. These oscillations may cause increased losses, power quality degradation, increased EMI and converter overloading [39]. The subsequent work can leverage this dissertation to further characterize how oscillations can persist and propagate an islanded microgrid with inverters having different controls such as droop control, VSG control, and PQ control. This will confer additional understanding into the mechanisms behind the successful tuning of feedback controllers and also provide a framework for mitigation.

- After determining the source of the disturbance, system operator implements the closed loop controls to bring back the system towards stability. However, if the system stability does not improve after implementing closed loop controls and the cascading failure scenario initiates, system operator still have an option to implement controlled islanding to save the system from blackout. To ensure transient stability while implementing controlled islanding, coherency of the generating units is very critical. To illustrate it, an oscillation originating disturbance is applied at node $l_{645-L V 1}$ in IEEE 13-node distribution system operating in islanded mode. Inverter terminal voltage magnitude is measured and shown in Fig. 5.1. It clearly illustrates the formation of two non-coherent generating units groups (DG1 : 645_LV1,DG2 : 645_LV2) and (DG3 : 634_LV1,DG4 : 634_LV2). 


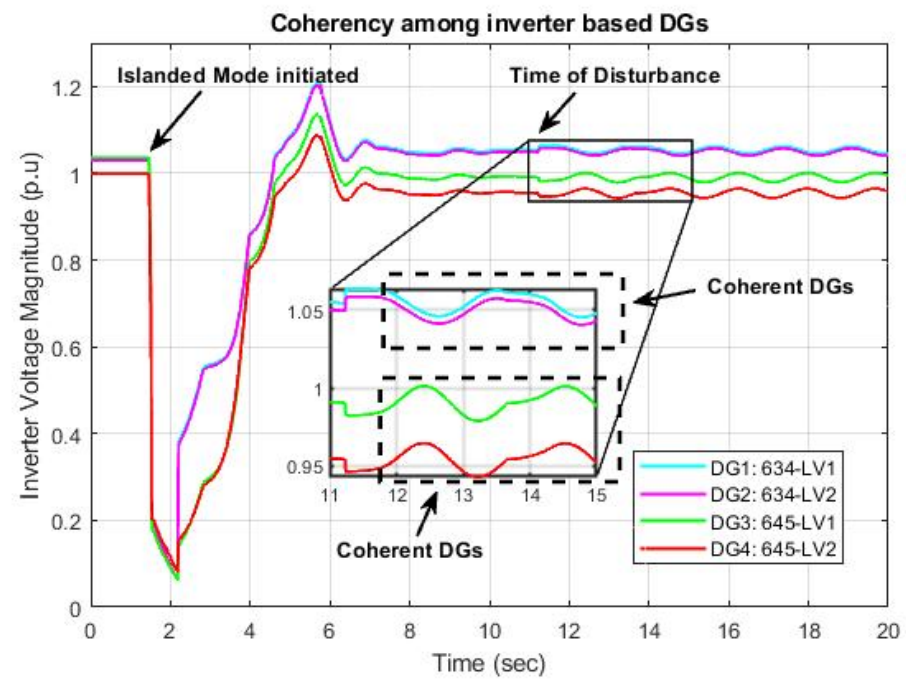

Fig. 5.1 Coherency among inverter based DGs

This disseetaton can be leveraged to use structural characterisitc based coherency identification framework to evaluate and analyze the coherency between inverter based distributed generators for adaptive emergency response. 


\section{LIST OF PUBLICATIONS}

1. Hasan Ul Banna, Zhe Yu, Di Shi, Zhiwei Wang, Dawei Su, Chunlei Xu, Sarika Khushalani Solanki, and Jignesh M. Solanki. "Online coherence identification using dynamic time warping for controlled islanding." Journal of Modern Power Systems and Clean Energy 7, no. 1 (2019): 38-54.

2. Hasan Ul Banna, Sarika Khushalani Solanki and Jignesh Solanki. "Data Driven Disturbance Source Identification for Power System Oscillations Using Credibility Search Ensemble Learning.” IET Smart Grid (2019).

3. Hasan Ul Banna, Vishal Verma, Sarika Khushalani Solanki, and Jignesh M. Solanki. $" \mu$-PMU Measurement based Oscillation SourceIdentification Using Innovative Ensemble Learningin Islanded Microgrid.” Ready for submission

4. Hasan Ul Banna, Sarika Khushalani Solanki, and Jignesh Solanki. "Structural Characteristic Measure based Data Driven Coherence Identification of Generators using PMU Measurements.” In 2019 IEEE Power Energy Society General Meeting (PESGM), pp. 1-5. IEEE, 2019.

5. Hasan Ul Banna, Sarika Khushalani Solanki, and Jignesh Solanki. "Spatial and Temporal Redundancy Removal in Disturbance Events Recorded by Phasor Measurement Units.” In 2018 IEEE Power Energy Society General Meeting (PESGM), pp. 1-5. IEEE, 2018.

6. Hasan U1 Banna, Deepak Tiwari, Sarika Khushalani Solanki and Jignesh Solanki, "Load variance minimization for coordinated PHEV charging in microgrid," 2016 North American Power Symposium (NAPS), Denver, CO, 2016, pp. 1-5. 


\section{REFERENCES}

[1] Yanzhu Ye and Yilu Liu. Monitoring power system disturbances based on distribution-level phasor measurements. In 2012 IEEE PES Innovative Smart Grid Technologies (ISGT), pages 1-8. IEEE, 2012.

[2] Reliability Guideline. Forced oscillation monitoring and mitigation. North American Electric Reliability Corporation, 2017.

[3] Nagaraju Pogaku, Milan Prodanovic, and Timothy C Green. Modeling, analysis and testing of autonomous operation of an inverter-based microgrid. IEEE Transactions on power electronics, 22(2):613-625, 2007.

[4] Pouyan Pourbeik, Prabha S Kundur, and Carson W Taylor. The anatomy of a power grid blackout-root causes and dynamics of recent major blackouts. IEEE Power and Energy Magazine, 4(5):22-29, 2006.

[5] Meir Klein, Graham J Rogers, and Prabha Kundur. A fundamental study of inter-area oscillations in power systems. IEEE Transactions on power systems, 6(3):914-921, 1991.

[6] Paul L Dandeno, Alex N Karas, Kenneth R McClymont, and Wilfred Watson. Effect of high-speed rectifier excitation systems on generator stability limits. IEEE Transactions on Power Apparatus and Systems, (1):190-201, 1968.

[7] Sudipta Ghosh and Nilanjan Senroy. The localness of electromechanical oscillations in power systems. International Journal of Electrical Power and Energy Systems, 42(1):306-313, 2012.

[8] ISO New England. Synchrophasor infrastructure and data utilization in the iso new england transmission region. Final Project Description in Smart Grid Investment Grant in, 2009.

[9] Hasan Ul Banna, Alvaro Luna, Shaoqing Ying, Hamidreza Ghorbani, and Pedro Rodriguez. Impacts of wind energy in-feed on power system small signal stability. In 2014 International Conference on Renewable Energy Research and Application (ICRERA), pages 615-622. IEEE, 2014. 
[10] Hasan Ul Banna, Alvaro Luna, Pedro Rodriguez, Ana Cabrera, Hamidreza Ghorbani, and Shaoqing Ying. Performance analysis of conventional pss and fuzzy controller for damping power system oscillations. In 2014 International Conference on Renewable Energy Research and Application (ICRERA), pages 229-234. IEEE, 2014.

[11] S Arash Nezam Sarmadi and Vaithianathan Venkatasubramanian. Inter-area resonance in power systems from forced oscillations. IEEE Transactions on Power Systems, 31(1):378-386, 2016.

[12] Lei Chen, Yong Min, and Wei Hu. An energy-based method for location of power system oscillation source. IEEE Transactions on Power Systems, 28(2):828-836, 2013.

[13] Prabha Kundur, Neal J Balu, and Mark G Lauby. Power system stability and control, volume 7. McGraw-hill New York, 1994.

[14] N Rostamkolai, RJ Piwko, and AS Matusik. Evaluation of the impact of a large cyclic load on the lilco power system using time simulation and frequency domain techniques. IEEE transactions on power systems, 9(3):1411-1416, 1994.

[15] Luigi Vanfretti, Sebastian Bengtsson, Vedran S Perić, and Jan O Gjerde. Effects of forced oscillations in power system damping estimation. In 2012 IEEE International Workshop on Applied Measurements for Power Systems (AMPS) Proceedings, pages 1-6. IEEE, 2012.

[16] Kyaw Myo Lin. Small signal stability assessment of mepe test system in free and open source software. International Journal of Electrical, Computer, Energetic, Electronic and Communication Engineering, 8:1707-1715, 012014.

[17] X. Wang and K. Turitsyn. Data-driven diagnostics of mechanism and source of sustained oscillations. IEEE Transactions on Power Systems, 31(5):4036-4046, Sep. 2016.

[18] G. Ghanavati, P. D. H. Hines, and T. I. Lakoba. Identifying useful statistical indicators of proximity to instability in stochastic power systems. IEEE Transactions on Power Systems, 31(2):1360-1368, March 2016.

[19] S. A. N. Sarmadi and V. Venkatasubramanian. Inter-area resonance in power systems from forced oscillations. IEEE Transactions on Power Systems, 31(1):378386, Jan 2016. 
[20] L. Chen, Y. Min, and W. Hu. An energy-based method for location of power system oscillation source. IEEE Transactions on Power Systems, 28(2):828-836, May 2013.

[21] J. Ma, P. Zhang, H. Fu, B. Bo, and Z. Dong. Application of phasor measurement unit on locating disturbance source for low-frequency oscillation. IEEE Transactions on Smart Grid, 1(3):340-346, Dec 2010.

[22] Y Li, Y Huang, J Liu, W Yao, and J Wen. Power system oscillation source location based on damping torque analysis. Dianli Xitong Baohu yu Kongzhi/Power System Protection and Control, 43:84-91, 072015.

[23] Yi Gao, Di-chen Liu, Guan-bin Huang, and Qiu-yun Shi. Locating method of disturbance source of forced power oscillation based on prony anyasis. pages 1-4, 092012.

[24] T. R. Nudell and A. Chakrabortty. Graph-theoretic methods for measurementbased input localization in large networked dynamic systems. IEEE Transactions on Automatic Control, 60(8):2114-2128, Aug 2015.

[25] M. A. M. Ariff and B. C. Pal. Coherency identification in interconnected power system-an independent component analysis approach. IEEE Transactions on Power Systems, 28(2):1747-1755, May 2013.

[26] M. Kazerooni, H. Zhu, and T. J. Overbye. Literature review on the applications of data mining in power systems. In 2014 Power and Energy Conference at Illinois (PECI), pages 1-8, Feb 2014.

[27] Muhammad Hussnain Riaz, Muhammad Zeeshan, Tariq Kamal, S Aamir Hussain Shah, et al. Demand side management using different energy conservation techniques. In 2017 International Multi-topic Conference (INMIC), pages 1-4. IEEE, 2017.

[28] J. Shin, B. Yi, Y. Kim, H. Lee, and K. H. Ryu. Spatiotemporal load-analysis model for electric power distribution facilities using consumer meter-reading data. IEEE Transactions on Power Delivery, 26(2):736-743, April 2011.

[29] J. N. Bank, O. A. Omitaomu, S. J. Fernandez, and Y. Liu. Visualization and classification of power system frequency data streams. In 2009 IEEE International Conference on Data Mining Workshops, pages 650-655, Dec 2009.

[30] P. G. V. Axelberg, I. Y. Gu, and M. H. J. Bollen. Support vector machine for classification of voltage disturbances. IEEE Transactions on Power Delivery, 22(3):1297-1303, July 2007. 
[31] M. He, V. Vittal, and J. Zhang. Online dynamic security assessment with missing pmu measurements: A data mining approach. IEEE Transactions on Power Systems, 28(2):1969-1977, May 2013.

[32] K. Seethalekshmi, S. N. Singh, and S. C. Srivastava. A classification approach using support vector machines to prevent distance relay maloperation under power swing and voltage instability. IEEE Transactions on Power Delivery, 27(3):1124-1133, July 2012.

[33] S. Pan, T. Morris, and U. Adhikari. Developing a hybrid intrusion detection system using data mining for power systems. IEEE Transactions on Smart Grid, 6(6):3104-3113, Nov 2015.

[34] C. Liu, K. Sun, Z. H. Rather, Z. Chen, C. L. Bak, P. Thøgersen, and P. Lund. A systematic approach for dynamic security assessment and the corresponding preventive control scheme based on decision trees. IEEE Transactions on Power Systems, 29(2):717-730, March 2014.

[35] K. Sun, S. Likhate, V. Vittal, V. S. Kolluri, and S. Mandal. An online dynamic security assessment scheme using phasor measurements and decision trees. IEEE Transactions on Power Systems, 22(4):1935-1943, Nov 2007.

[36] Hasan Ul Banna, Deepak Tiwari, Sarika K Solanki, and Jignesh Solanki. Load variance minimization for coordinated phev charging in microgrid. In 2016 North American Power Symposium (NAPS), pages 1-5. IEEE, 2016.

[37] J. Sun. Small-signal methods for ac distributed power systems-a review. IEEE Transactions on Power Electronics, 24(11):2545-2554, Nov 2009.

[38] Allal M. Bouzid, Josep M. Guerrero, Ahmed Cheriti, Mohamed Bouhamida, Pierre Sicard, and Mustapha Benghanem. A survey on control of electric power distributed generation systems for microgrid applications. Renewable and Sustainable Energy Reviews, 44:751 - 766, 2015.

[39] H. Saghafi, H. Karshenas, A. Bakhshai, and P. Jain. Power oscillation damping in standalone microgrids using integrated series compensator. In 2012 TwentySeventh Annual IEEE Applied Power Electronics Conference and Exposition (APEC), pages 1940-1945, Feb 2012.

[40] Slava Maslennikov, Bin Wang, and Eugene Litvinov. Dissipating energy flow method for locating the source of sustained oscillations. International Journal of Electrical Power Energy Systems, 88:55-62, 062017. 
[41] Samuel C Chevalier, Petr Vorobev, and Konstantin Turitsyn. Using effective generator impedance for forced oscillation source location. IEEE Transactions on Power Systems, 33(6):6264-6277, 2018.

[42] WANG Bin and SUN Kai. Location methods of oscillation sources in power systems: a survey. Journal of Modern Power Systems and Clean Energy, 5(2):151159, 2017.

[43] Slava Maslennikov, Bin Wang, Qiang Zhang, Eugene Litvinov, et al. A test cases library for methods locating the sources of sustained oscillations. In 2016 IEEE Power and Energy Society General Meeting (PESGM), pages 1-5. IEEE, 2016.

[44] Han Wu, Qingang Duan, and Jin Ma. Disturbance source self-diagnosis of the smart grid. In 2012 Spring Congress on Engineering and Technology, pages 1-4. IEEE, 2012.

[45] Jin Ma, Pu Zhang, Hong-jun Fu, Bo Bo, and Zhao-yang Dong. Application of phasor measurement unit on locating disturbance source for low-frequency oscillation. IEEE Transactions on Smart Grid, 1(3):340-346, 2010.

[46] Lei Chen, Fei Xu, Yong Min, Maohai Wang, and Wei Hu. Transient energy dissipation of resistances and its effect on power system damping. International Journal of Electrical Power and Energy Systems, 91:201-208, 2017.

[47] N Al-Ashwal, D Wilson, and M Parashar. Identifying sources of oscillations using wide area measurements. In Proceedings of the CIGRE US National Committee 2014 grid of the future symposium, Houston, volume 19, 2014.

[48] Thomas R Nudell and Chakrabortty Aranya. Graph-theoretic methods for measurement-based input localization in large networked dynamic systems. IEEE Transactions on Automatic Control, 60(8):2114-2128, 2015.

[49] R. Azim, Y. Zhu, H. A. Saleem, K. Sun, F. Li, D. Shi, and R. Sharma. A decision tree based approach for microgrid islanding detection. In 2015 IEEE Power Energy Society Innovative Smart Grid Technologies Conference (ISGT), pages 1-5, Feb 2015.

[50] F. R. Gomez, A. D. Rajapakse, U. D. Annakkage, and I. T. Fernando. Support vector machine-based algorithm for post-fault transient stability status prediction using synchronized measurements. IEEE Transactions on Power Systems, 26(3):1474-1483, Aug 2011. 
[51] Jiawei Han, Micheline Kamber, and Jian Pei. 1 - introduction. In Jiawei Han, Micheline Kamber, and Jian Pei, editors, Data Mining (Third Edition), The Morgan Kaufmann Series in Data Management Systems, pages 1 - 38. Morgan Kaufmann, Boston, third edition edition, 2012.

[52] Zhizhong Li and Dahua Lin. Integrating specialized classifiers based on continuous time markov chain. In Proceedings of the 26th International Joint Conference on Artificial Intelligence, IJCAI'17, pages 2244-2251. AAAI Press, 2017.

[53] Y. Zhu, R. Azim, H. A. Saleem, K. Sun, D. Shi, and R. Sharma. Microgrid security assessment and islanding control by support vector machine. In 2015 IEEE Power Energy Society General Meeting, pages 1-5, July 2015.

[54] TF.

[55] S. Maslennikov, B. Wang, Q. Zhang, a. Ma, a. Luo, a. Sun, and E. Litvinov. A test cases library for methods locating the sources of sustained oscillations. In 2016 IEEE Power and Energy Society General Meeting (PESGM), pages 1-5, July 2016.

[56] Ieee standard for synchrophasors for power systems. IEEE Std C37.118-2005 (Revision of IEEE Std 1344-1995), pages 1-65, March 2006.

[57] Sandip Sharma, Shun-Hsien Huang, and NDR Sarma. System inertial frequency response estimation and impact of renewable resources in ercot interconnection. In 2011 IEEE power and energy society general meeting, pages 1-6. IEEE, 2011.

[58] Miguel Castilla, Luis García de Vicuña, and Jaume Miret. Control of power converters in ac microgrids. In Microgrids Design and Implementation, pages 139-170. Springer, 2019.

[59] Ritwik Majumder. Some aspects of stability in microgrids. IEEE Transactions on power systems, 28(3):3243-3252, 2013.

[60] Qing-Chang Zhong and George Weiss. Synchronverters: Inverters that mimic synchronous generators. IEEE Transactions on Industrial Electronics, 58(4):1259-1267, 2010.

[61] Jia Liu, Yushi Miura, and Toshifumi Ise. Comparison of dynamic characteristics between virtual synchronous generator and droop control in inverter-based distributed generators. IEEE Transactions on Power Electronics, 31(5):3600-3611, 2015. 
[62] Salvatore D'Arco, Jon Are Suul, and Olav B Fosso. Control system tuning and stability analysis of virtual synchronous machines. In 2013 IEEE Energy Conversion Congress and Exposition, pages 2664-2671. IEEE, 2013.

[63] WH Kersting. A comprehensive distribution test feeder. In IEEE PES TandD 2010, pages 1-4. IEEE, 2010.

[64] P Nemenyi. Distribution-free multiple comparisons (doctoral dissertation, princeton university). Ann Arbor: University Microfilms, (64-6278), 1963.

[65] Jing Ma, Dong Zhao, Yaqi Shen, and AG Phadke. Research on positioning method of low frequency oscillating source in dfig-integrated system with virtual inertia control. IEEE Transactions on Sustainable Energy, 2019.

[66] Haibo You, Vijay Vittal, and Xiaoming Wang. Slow coherency-based islanding. IEEE Transactions on Power Systems, 19(1):483-491, 2004.

[67] SB Yusof, GJ Rogers, and RTH Alden. Slow coherency based network partitioning including load buses. IEEE Transactions on Power Systems, 8(3):1375-1382, 1993.

[68] Hasan Ul Banna, Sarika Khushalani Solanki, and Jignesh Solanki. Structural characteristic measure based data driven coherence identification of generators using pmu measurements. In 2019 IEEE Power and Energy Society General Meeting (PESGM), pages 1-5. IEEE, 2019.

[69] Lei Ding, Francisco M Gonzalez-Longatt, Peter Wall, and Vladimir Terzija. Two-step spectral clustering controlled islanding algorithm. IEEE Transactions on Power Systems, 28(1):75-84, 2012.

[70] A Muir and J Lopatto. Final report on the august 14, 2003 blackout in the united states and canada: Causes and recommendations. 2004.

[71] O. Gomez and M. A. Rios. Real time identification of coherent groups for controlled islanding based on graph theory. IET Generation, Transmission Distribution, 9(8):748-758, 2015.

[72] and V. Vittal and G. T. Heydt. Tracing generator coherency indices using the continuation method: a novel approach. IEEE Transactions on Power Systems, 20(3):1510-1518, Aug 2005.

[73] S. B. Yusof, G. J. Rogers, and R. T. H. Alden. Slow coherency based network partitioning including load buses. IEEE Transactions on Power Systems, 8(3):13751382, Aug 1993. 
[74] B. Yang, V. Vittal, and G. T. Heydt. Slow-coherency-based controlled islanding - a demonstration of the approach on the august 14, 2003 blackout scenario. IEEE Transactions on Power Systems, 21(4):1840-1847, Nov 2006.

[75] J. H. Chow. New algorithms for slow coherency aggregation of large power systems. Institute for Mathematics and Its Applications, 64:95, 1995.

[76] Y. Ota, H. Ukai, K. Nakamura, and H. Fujita. Pmu based midterm stability evaluation of wide-area power system. In IEEE/PES Transmission and Distribution Conference and Exhibition, volume 3, pages 1676-1680 vol.3, Oct 2002.

[77] M. Jonsson, M. Begovic, and J. Daalder. A new method suitable for realtime generator coherency determination. IEEE Transactions on Power Systems, 19(3):1473-1482, Aug 2004.

[78] Mattias Jonsson, Miroslav Begovic, and Jaap Daalder. A new method suitable for real-time generator coherency determination. IEEE Transactions on Power Systems, 19(3):1473-1482, 2004.

[79] Krishna K Anaparthi, Balarko Chaudhuri, Nina F Thornhill, and Bikash C Pal. Coherency identification in power systems through principal component analysis. IEEE transactions on power systems, 20(3):1658-1660, 2005.

[80] Fahd Hashiesh, Hossam E Mostafa, Ibrahim Helal, and Mohamed M Mansour. Determination of generators coherent groups based on synchrophasors using bioinformatics toolbox. In 2011 IEEE PES Conference on Innovative Smart Grid Technologies-Middle East, pages 1-7. IEEE, 2011.

[81] MAM Ariff and Bikash C Pal. Coherency identification in interconnected power system - an independent component analysis approach. IEEE Transactions on Power Systems, 28(2):1747-1755, 2013.

[82] M Abd-EI-Aal Abd-EI, ID Helal, M Anward Hassan Omar, et al. Multi-machine power system dynamic equivalents using artificial intelligence (ann). In 2006 Eleventh International Middle East Power Systems Conference, volume 1, pages 197-207. IEEE, 2006.

[83] Haozhou Wang, Han Su, Kai Zheng, Shazia Sadiq, and Xiaofang Zhou. An effectiveness study on trajectory similarity measures. In Proceedings of the TwentyFourth Australasian Database Conference-Volume 137, pages 13-22. Australian Computer Society, Inc., 2013. 
[84] Zhang Zhang, Kaiqi Huang, Tieniu Tan, et al. Comparison of similarity measures for trajectory clustering in outdoor surveillance scenes. In $\operatorname{ICPR}(3)$, pages 11351138. Citeseer, 2006.

[85] Félix Iglesias and Wolfgang Kastner. Analysis of similarity measures in times series clustering for the discovery of building energy patterns. Energies, 6(2):579597, 2013.

[86] MR Aghamohammadi and SM Tabandeh. A new approach for online coherency identification in power systems based on correlation characteristics of generators rotor oscillations. International Journal of Electrical Power and Energy Systems, 83:470-484, 2016.

[87] Zhenzhi LIN, Fushuan WEN, Junhua ZHAO, and Yusheng XUE. Controlled islanding schemes for interconnected power systems based on coherent generator group identification and wide-area measurements. Journal of Modern Power Systems and Clean Energy, 4(3):440-453, Jul 2016.

[88] Teofilo F. Gonzalez. Clustering to minimize the maximum intercluster distance. Theoretical Computer Science, 38:293 - 306, 1985.

[89] and and L. E. Jones and. Coherency and aggregation techniques incorporating rotor and voltage dynamics. IEEE Transactions on Power Systems, 19(2):10681075, May 2004.

[90] Lingling Fan and Yasser Wehbe. Extended kalman filtering based real-time dynamic state and parameter estimation using pmu data. Electric Power Systems Research, 103:168 - 177, 2013.

[91] Eamonn Keogh and Chotirat Ann Ratanamahatana. Exact indexing of dynamic time warping. Knowledge and Information Systems, 7(3):358-386, Mar 2005.

[92] Alon Efrat, Quanfu Fan, and Suresh Venkatasubramanian. Curve matching, time warping, and light fields: New algorithms for computing similarity between curves. Journal of Mathematical Imaging and Vision, 27(3):203-216, Apr 2007.

[93] Hui Ding, Goce Trajcevski, Peter Scheuermann, Xiaoyue Wang, and Eamonn Keogh. Querying and mining of time series data: Experimental comparison of representations and distance measures. Proc. VLDB Endow., 1(2):1542-1552, August 2008.

[94] Xiaoyue Wang, Abdullah Mueen, Hui Ding, Goce Trajcevski, Peter Scheuermann, and Eamonn Keogh. Experimental comparison of representation methods 
and distance measures for time series data. Data Mining and Knowledge Discovery, 26(2):275-309, Mar 2013.

[95] TF. https://www. mathworks.com/help/stats/kmeans.html.

[96] and G. W. Rosenwald and J. Jung and. Strategic power infrastructure defense. Proceedings of the IEEE, 93(5):918-933, May 2005.

[97] TF. http://www.docsdrive.com/pdfs/ansinet/jas/2009/ 2247-2255.pdf.

[98] Li Liu, Wenxin Liu, David A. Cartes, and Il-Yop Chung. Slow coherency and angle modulated particle swarm optimization based islanding of large-scale power systems. Advanced Engineering Informatics, 23(1):45 - 56, 2009.

[99] S. Najafi. Evaluation of interconnected power systems controlled islanding. In 2009 IEEE Bucharest PowerTech, pages 1-8, June 2009.

[100] Chen Shen, J.-Y Wu, Y Qiao, Q Lu, Q.-J Liu, and C Rehtanz. Studies on active splitting control of power systems. 26:1-6, 072006.

[101] A. Esmaeilian and M. Kezunovic. Prevention of power grid blackouts using intentional islanding scheme. IEEE Transactions on Industry Applications, 53(1):622-629, Jan 2017.

[102] Jairo Quirós-Tortós. Constrained spectral clustering-based methodology for intentional controlled islanding of large-scale power systems. IET Generation, Transmission Distribution, 9:31-42(11), January 2015.

[103] Xiang Wang, Buyue Qian, and Ian Davidson. On constrained spectral clustering and its applications. Data Mining and Knowledge Discovery, 28(1):1-30, Jan 2014.

[104] Harold W. Kuhn. Nonlinear programming: A historical view. SIGMAP Bull., (31):6-18, June 1982.

[105] TF. https://www.mathworks.com/help/stats/kmedoids.html.

[106] L. Ding, F. M. Gonzalez-Longatt, P. Wall, and V. Terzija. Two-step spectral clustering controlled islanding algorithm. IEEE Transactions on Power Systems, 28(1):75-84, Feb 2013.

[107] Arunabha Sen, Pavel Ghosh, Vijay Vittal, and Bo Yang. A new min-cut problem with application to electric power network partitioning. European Transactions on Electrical Power, 19(6):778-797. 
[108] Ian Davidson and S. S. Ravi. The complexity of non-hierarchical clustering with instance and cluster level constraints. Data Mining and Knowledge Discovery, 14(1):25-61, Feb 2007.

[109] Renato Cordeiro de Amorim and Christian Hennig. Recovering the number of clusters in data sets with noise features using feature rescaling factors. Information Sciences, 324:126 - 145, 2015.

[110] Peter J. Rousseeuw. Silhouettes: A graphical aid to the interpretation and validation of cluster analysis. Journal of Computational and Applied Mathematics, 20:53-65, 1987.

[111] Z. Lin, F. Wen, Y. Ding, and Y. Xue. Data-driven coherency identification for generators based on spectral clustering. IEEE Transactions on Industrial Informatics, 14(3):1275-1285, March 2018.

[112] M. Mahdi and V. M. I. Genc. A real-time self-healing methodology using modeland measurement-based islanding algorithms. IEEE Transactions on Smart Grid, 10(2):1195-1204, March 2019.

[113] Honglei Song, Junyong $\mathrm{Wu}$, and Kui Wu. A wide-area measurement systemsbased adaptive strategy for controlled islanding in bulk power systems. Energies, 7(4):2631-2657, 2014.

[114] M.R. Aghamohammadi and S.M. Tabandeh. A new approach for online coherency identification in power systems based on correlation characteristics of generators rotor oscillations. International Journal of Electrical Power Energy Systems, 83:470 - 484, 2016.

[115] TF. https://lib.dr.iastate.edu/cgi/viewcontent.cgi? article $=2602 \&$ context $=r t d$.

[116] Oscar Gomez. Real time identification of coherent groups for controlled islanding based on graph theory. IET Generation, Transmission Distribution, 9:748758(10), May 2015.

[117] M. Brown, M. Biswal, S. Brahma, S. J. Ranade, and H. Cao. Characterizing and quantifying noise in pmu data. In 2016 IEEE Power and Energy Society General Meeting (PESGM), pages 1-5, July 2016.

[118] Talha Iqbal, Hasan Ul Banna, and Muhammad Hussnain Riaz. Cyber intrusion detection through spatio-temporal correlation in optimal power flow problem. In 2018 International Conference on Engineering and Emerging Technologies (ICEET), pages 1-5. IEEE, 2018. 
[119] Talha Iqbal and Hasan Ul Banna. Control system design to automate 100kv impulse generator. International Journal of Scientific and Engineering Research (IJSER), 5(4):1329-1336, 2014.

[120] Ana Cabrera Tobar, Hasan Ul Banna, and Cosmin Koch-Ciobotaru. Scope of electrical distribution system architecture considering the integration of renewable energy in large and small scale. In IEEE PES Innovative Smart Grid Technologies, Europe, pages 1-7. IEEE, 2014.

[121] Arsham Iqbal, Ibrahim F Muhammad, Muhammad Faraz, Muhammad S Tariq, and Hasan-ul Banna. Economic analysis of a small hybrid power system. In 2015 Power Generation System and Renewable Energy Technologies (PGSRET), pages 1-5. IEEE, 2015.

[122] Ana K Cabrera, Hasan Ul Banna, Cosmin Koch-Ciobotarus, and Siddharta Ghosh. Optimization of an air conditioning unit according to renewable energy availability and user's comfort. In IEEE PES Innovative Smart Grid Technologies, Europe, pages 1-7. IEEE, 2014.

[123] T. Iqbal, A. Dehghan Banadaki, and A. Feliachi. Optimal voltage control using singular value decomposition of fast decoupled load flow jacobian. In 2017 North American Power Symposium (NAPS), pages 1-6, Sep. 2017.

[124] Hasan U1 Banna, Talha Iqbal, Ayesha Khan, and Zoupash Zahra. Generators coherency identification using relative correlation based clustering. In 2018 International Conference on Engineering and Emerging Technologies (ICEET), pages 1-5. IEEE, 2018.

[125] T. Iqbal. Secondary voltage control using singular value decomposition by discovering community structures in power networks, 2017.

[126] AM Miah. Study of a coherency-based simple dynamic equivalent for transient stability assessment. IET Generation, Transmission and Distribution, 5(4):405416, 2011.

[127] Tao Jiang, Hongjie Jia, Haoyu Yuan, Ning Zhou, and Fangxing Li. Projection pursuit: a general methodology of wide-area coherency detection in bulk power grid. IEEE Transactions on Power Systems, 31(4):2776-2786, 2016.

[128] LIN Zhenzhi, WEN Fushuan, ZHAO Junhua, and XUE Yusheng. Controlled islanding schemes for interconnected power systems based on coherent generator group identification and wide-area measurements. Journal of Modern Power Systems and Clean Energy, 4(3):440-453, 2016. 
[129] Hasan Ul Banna, Zhe Yu, Di Shi, Zhiwei Wang, Dawei Su, Chunlei Xu, Sarika Solanki, and Jignesh Solanki. Online coherence identification using dynamic time warping for controlled islanding. arXiv preprint arXiv:1708.06424, 2017.

[130] Talha Iqbal and Ali Feliachi. Discovering community structures in power system networks using voltage-reactive power sensitivity. In 2017 North American Power Symposium (NAPS), pages 1-6. IEEE, 2017.

[131] Talha Iqbal and Ali Feliachi. Decentralized voltage control using fast community detection algorithm and eigen decomposition. In 2019 IEEE Power and Energy Society General Meeting (PESGM), pages 1-5. IEEE, 2019.

[132] Hasan Ul Banna, Sarika Khushalani Solanki, and Jignesh Solanki. Spatial and temporal redundancy removal in disturbance events recorded by phasor measurement units. In 2018 IEEE Power and Energy Society General Meeting (PESGM), pages 1-5. IEEE, 2018.

[133] Hasan Ul Banna, Sarika Khushalani Solanki, and Jignesh Solanki. Data-driven disturbance source identification for power system oscillations using credibility search ensemble learning. IET Smart Grid, 2(2):293-300, 2019.

[134] DN Joanes and CA Gill. Comparing measures of sample skewness and kurtosis. Journal of the Royal Statistical Society: Series D (The Statistician), 47(1):183189, 1998.

[135] Jens Feder. Fractals (physics of solids and liquids). New York: Plenu, 1988.

[136] Torsten Kleinow. Testing continuous time models in financial markets. 2002.

[137] Lawrence T DeCarlo. On the meaning and use of kurtosis. Psychological methods, 2(3):292, 1997.

[138] Steven M Pincus, Igor M Gladstone, and Richard A Ehrenkranz. A regularity statistic for medical data analysis. Journal of clinical monitoring, 7(4):335-345, 1991.

[139] Peter J Rousseeuw. Silhouettes: a graphical aid to the interpretation and validation of cluster analysis. Journal of computational and applied mathematics, 20:53-65, 1987.

[140] Renato Cordeiro de Amorim and Christian Hennig. Recovering the number of clusters in data sets with noise features using feature rescaling factors. Information Sciences, 324:126-145, 2015. 
[141] Zhenzhi Lin, Fushuan Wen, Yi Ding, and Yusheng Xue. Data-driven coherency identification for generators based on spectral clustering. IEEE Transactions on Industrial Informatics, 14(3):1275-1285, 2018.

[142] Mohammed Mahdi and VM Istemihan Genc. A real-time self-healing methodology using model and measurement based islanding algorithms. IEEE Transactions on Smart Grid, 2017.

[143] Allal M. Bouzid, Josep M. Guerrero, Ahmed Cheriti, Mohamed Bouhamida, Pierre Sicard, and Mustapha Benghanem. A survey on control of electric power distributed generation systems for microgrid applications. Renewable and Sustainable Energy Reviews, 44(C):751-766, 2015. 
Appendices 


\section{Appendix A}

\section{Example: DTW based Similarity Evaluation}

A simple example to further clarify the formulation and implementation of DTW algorithm is presented here. Consider two trajectories, $\operatorname{tr} 1(\mathrm{t})=-0.60,-0.65,-0.71,-0.58$, $-0.17,-0.77,1.94$ and $\operatorname{tr} 2(\mathrm{t})=-0.87,-0.84,-0.85,-0.82,-0.23,1.95,1.360 .60,-0.29$. First local distance measures, between each pair of trajectories as formulated in (3.1), are calculated. A matrix of size 107 is obtained as shown in Fig.A.1. Then starting from element $(1,1)$, multiple summing paths can be traced to reach the final cell $(10,7)$ as described in (3.2). Out of these possible summing paths, an optimal path which would sum up to minimum distance will represent the DTW distance between trajectories $\operatorname{tr} 1(\mathrm{t})$ and tr2(t) as expressed in (3.3) and shown in Fig. A.1.

\begin{tabular}{|c|c|c|c|c|c|c|c|}
\hline 10 & 0.0961 & 0.1296 & 0.1764 & 0.0841 & 0.0144 & 1.1236 & 4.9729 \\
\hline 9 & 0.3600 & 0.4225 & 0.5041 & 0.3364 & 0.0289 & 0.5929 & 3.7636 \\
\hline 8 & 1.4400 & 1.5625 & 1.7161 & 1.3924 & 0.5929 & 0.0289 & 1.7956 \\
\hline 7 & 3.8416 & 4.0401 & 4.2849 & 3.7636 & 2.3409 & 0.3481 & 0.3364 \\
\hline 6 & 6.5025 & 6.7600 & 7.0756 & 6.4009 & 4.4944 & 1.3924 & 0.0001 \\
\hline 5 & 0.1369 & 0.1764 & 0.2304 & 0.1225 & 0.0036 & 1.0000 & 4.7089 \\
\hline 4 & 0.0484 & 0.0289 & 0.0121 & 0.0576 & 0.4225 & 2.5281 & 7.617 \\
\hline 3 & 0.0625 & 0.0400 & 0.0196 & 0.0729 & 0.4624 & 2.6244 & 7.7841 \\
\hline 2 & 0.0576 & 0.0861 & 0.0169 & 0.0676 & 0.4489 & 2.5921 & 7.7284 \\
\hline 1 & 0.0729 & 0.0484 & 0.0256 & 0.0841 & 0.4900 & 2.6896 & 7.8961 \\
\hline \multirow{2}{*}{} & 1 & 2 & 3 & 4 & 5 & 6 & 7 \\
\cline { 2 - 7 }
\end{tabular}

Fig. A.1 An optimal path formation through a matrix of local distance measures 